\title{
Controllability of two coupled wave equations on a compact manifold
}

\author{
Belhassen Dehman* Jérôme Le Rousseauł and Matthieu Léautaud ${ }^{\ddagger}$
}

July 19, 2012

\begin{abstract}
We consider the exact controllability problem on a compact manifold $\Omega$ for two coupled wave equations, with a control function acting on one of them only. Action on the second wave equation is obtained through a coupling term.

First, when the two waves propagate with the same speed, we introduce the time $T_{\omega \rightarrow \mathcal{O} \rightarrow \omega}$ for which all geodesics traveling in $\Omega$ go through the control region $\omega$, then through the coupling region $\mathcal{O}$, and finally come back in $\omega$. We prove that the system is controllable if and only if both $\omega$ and $\mathcal{O}$ satisfy the Geometric Control Condition and the control time is larger than $T_{\omega \rightarrow \mathcal{O} \rightarrow \omega}$.

Second, we prove that the associated HUM control operator is a pseudodifferential operator and we exhibit its principal symbol.

Finally, if the two waves propagate with different speeds, we give sharp sufficient controllability conditions on the functional spaces, the geometry of the sets $\omega$ and $\mathcal{O}$, and the minimal time.
\end{abstract}

\section{Keywords}

Wave equation, system, microlocal defect measures, controllability, HUM operator.

\section{Contents}

1 Introduction and main result $\quad \mathbf{2}$

1.1 Setting and motivation . . . . . . . . . . . . . . . . . . . . . . . 2

1.2 Main results . . . . . . . . . . . . . . . . . . . . . . . . . . 4

1.3 Comments and outline ........................... 6

2 Preliminary remarks, definitions and notation 8

2.1 Symbols, operators and measures on the cosphere bundle . . . . . . . . . . . . 8

2.2 Some geometric facts . . . . . . . . . . . . . . . . . . . . . . . . 11

2.3 Reformulation of the system in symmetric spaces . . . . . . . . . . . . . . . 12

3 Observability for $T>T_{\omega \rightarrow \mathcal{O} \rightarrow \omega} \quad 13$

3.1 A relaxed observability inequality . . . . . . . . . . . . . . . . . . . . 14

3.2 End of the proof of Proposition 3.1 . . . . . . . . . . . . . . . . . 17

4 Lack of observability for $T<T_{\omega \rightarrow \mathcal{O} \rightarrow \omega}$

*Département de Mathématiques, Faculté des sciences de Tunis, Université de Tunis El Manar, 2092 El Manar, Tunisia. e-mail: Belhassen.Dehman@fst.rnu.tn

${ }^{\dagger}$ Laboratoire de Mathématiques - Analyse, Probabilités, Modélisation - Orléans, CNRS UMR 6628, Fédération Denis-Poisson, FR CNRS 2964, Université d'Orléans, B.P. 6759, 45067 Orléans cedex 2, France. e-mail: jlr@univ-orleans.fr

‡Université Paris-Sud 11, Mathématiques, Bâtiment 425, 91405 Orsay Cedex, France. e-mail: matthieu.leautaud@math.u-psud.fr 
5 The Hilbert Uniqueness Method and the HUM operator 23

5.1 Controllability and observability for cascade Systems . . . . . . . . . . . . . . . 23

5.2 The HUM operator . . . . . . . . . . . . . . . . . . . . . 25

5.3 Microlocal characterization of the HUM operator . . . . . . . . . . . . . . . . . 26

6 Coupled waves with different speeds $\quad 39$

A 1-smoothing properties $\quad 45$

$\begin{array}{ll}\text { B Proofs of some technical results } & 47\end{array}$

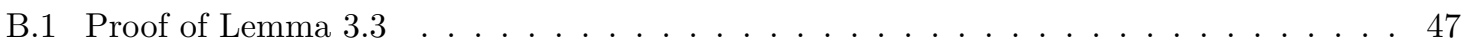

B.2 Proof of Lemma $4.3 \ldots \ldots \ldots \ldots \ldots \ldots$

\section{Introduction and main result}

\subsection{Setting and motivation}

Let $(\Omega, g)$ be a $\mathscr{C}^{\infty}$ compact connected $n$-dimensional Riemannian manifold without boundary. We denote by $\Delta$ the (negative) Laplace-Beltrami operator on $\Omega$ for the metric $g$, and $P=P\left(t, x, \partial_{t}, \partial_{x}\right)=$ $\partial_{t}^{2}-\Delta$ denotes the d'Alembert operator (or wave operator) on the manifold $\mathbb{R} \times \Omega$. We take two smooth functions $b_{\omega}$ and $b$ on $\Omega$. We consider the controllability problem for the system of coupled wave equations

$$
\begin{cases}P u_{1}+b(x) u_{2}=0 & \text { in }(0, T) \times \Omega, \\ P u_{2}=b_{\omega}(x) f & \text { in }(0, T) \times \Omega .\end{cases}
$$

Here, the state of the system is $\left(u_{1}, u_{2}, \partial_{t} u_{1}, \partial_{t} u_{2}\right)$ and $f$ is our control function, with possible action on the set $\left\{b_{\omega} \neq 0\right\}$. Taking zero initial data, together with a forcing term $f \in L^{2}((0, T) \times \Omega)$ ), the associated solution of (1.1) lies for any time in the space $H^{2}(\Omega) \times H^{1}(\Omega) \times H^{1}(\Omega) \times L^{2}(\Omega)$ as $u_{2} \in L^{2}\left(0, T ; H^{1}(\Omega)\right)$. Hence, there is a gain of regularity for the uncontrolled variable $u_{1}$ (see also [AB03, ABL11, ABL12]).

In this context, the adapted control problem is given by the following definition. Because of the linearity and the reversibility of the system, the three statements are equivalent.

Definition 1.1. We say that System (1.1) is controllable in time $T>0$ if one of the following (equivalent) assertions is satisfied:

- (Exact controllability) For any initial data $\left(u_{1}^{0}, u_{2}^{0}, u_{1}^{1}, u_{2}^{1}\right) \in H^{2}(\Omega) \times H^{1}(\Omega) \times H^{1}(\Omega) \times L^{2}(\Omega)$ and any target $\left(\tilde{u}_{1}^{0}, \tilde{u}_{2}^{0}, \tilde{u}_{1}^{1}, \tilde{u}_{2}^{1}\right) \in H^{2}(\Omega) \times H^{1}(\Omega) \times H^{1}(\Omega) \times L^{2}(\Omega)$ there exists a control function $f \in L^{2}((0, T) \times \Omega)$ such that the solution of (1.1) issued from $\left.\left(u_{1}, u_{2}, \partial_{t} u_{1}, \partial_{t} u_{2}\right)\right|_{t=0}=$ $\left(u_{1}^{0}, u_{2}^{0}, u_{1}^{1}, u_{2}^{1}\right)$, satisfies $\left.\left(u_{1}, u_{2}, \partial_{t} u_{1}, \partial_{t} u_{2}\right)\right|_{t=T}=\left(\tilde{u}_{1}^{0}, \tilde{u}_{2}^{0}, \tilde{u}_{1}^{1}, \tilde{u}_{2}^{1}\right)$

- (Null-controllability) For any initial data $\left(u_{1}^{0}, u_{2}^{0}, u_{1}^{1}, u_{2}^{1}\right) \in H^{2}(\Omega) \times H^{1}(\Omega) \times H^{1}(\Omega) \times L^{2}(\Omega)$, there exists a control function $f \in L^{2}((0, T) \times \Omega)$ such that the solution of (1.1) associated to the initial data $\left.\left(u_{1}, u_{2}, \partial_{t} u_{1}, \partial_{t} u_{2}\right)\right|_{t=0}=\left(u_{1}^{0}, u_{2}^{0}, u_{1}^{1}, u_{2}^{1}\right)$ satisfies $\left.\left(u_{1}, u_{2}, \partial_{t} u_{1}, \partial_{t} u_{2}\right)\right|_{t=T}=$ $(0,0,0,0)$;

- (Controllability from zero) For any target $\left(\tilde{u}_{1}^{0}, \tilde{u}_{2}^{0}, \tilde{u}_{1}^{1}, \tilde{u}_{2}^{1}\right) \in H^{2}(\Omega) \times H^{1}(\Omega) \times H^{1}(\Omega) \times L^{2}(\Omega)$, there exists a control function $f \in L^{2}((0, T) \times \Omega)$ such that the solution of (1.1) starting from rest $\left.\left(u_{1}, u_{2}, \partial_{t} u_{1}, \partial_{t} u_{2}\right)\right|_{t=0}=(0,0,0,0)$ satisfies $\left.\left(u_{1}, u_{2}, \partial_{t} u_{1}, \partial_{t} u_{2}\right)\right|_{t=T}=\left(\tilde{u}_{1}^{0}, \tilde{u}_{2}^{0}, \tilde{u}_{1}^{1}, \tilde{u}_{2}^{1}\right)$;

For most results proved in this article, we shall assume that the function $b$ is non-negative on $\Omega$, and denote by $\omega=\left\{b_{\omega} \neq 0\right\}$ the control set and by $\mathcal{O}=\{b \neq 0\}$ the coupling set (which is the indirect control set for the first equation in (1.1)). 
A natural necessary and sufficient condition to obtain controllability for wave equations is to assume that the control set satisfies the Geometric Control Condition (GCC) defined in [RT74, BLR92]. For $\omega \subset \Omega$ and $T>0$, we shall say that $(\omega, T)$ satisfies GCC if every geodesic traveling at speed one in $\Omega$ meets $\omega$ in a time $t<T$. We say that $\omega$ satisfies GCC if there exists $T>0$ such that $(\omega, T)$ satisfies GCC. We also set $T_{\omega}=\inf \{T>0,(\omega, T)$ satisfies GCC $\}$.

Note that in the situation of System (1.1), a necessary condition is that both sets $\omega$ and $\mathcal{O}$ satisfy GCC (otherwise one of the two equations is not controllable). If $\omega$ does not satisfy GCC, even the second equation of (1.1) is not controllable (see [BLR92, Bur97a] for a single wave equation). If $\mathcal{O}$ does not satisfy GCC, the first equation of (1.1) is not controllable for the same reason.

The controllability problem for systems like (1.1) has already been addressed in [ABL11, ABL12, RdT11]. In the first two articles, and in the context of symmetric systems, it is proved that controllability holds in large time under optimal geometric conditions on the sets $\omega$ and $\mathcal{O}$. However, the minimal time given in these articles depends upon all parameters of the problem (i.e. $b$ and $b_{\omega}$ ). In the situation of System (1.1), it seems natural that the control time should depend only on the geometry of the sets $\Omega, \omega$ and $\mathcal{O}$, as it is the case for a single wave equation. In [RdT11], the authors study System (1.1) in the one dimensional torus. Following [Dág06], they obtain a sharper estimate on the control time than in [ABL11, ABL12] (in particular, it depends only on the sets $\Omega, \omega$ and $\mathcal{O}$ ). Yet, it is not optimal in general.

We provide some motivations for considering control systems like (1.1).

Controllability of physical systems. Several physical systems can be described by coupled partial deifferential equations: Elasticity, Thermoelasticity, Elecromagnetism, plate systems,... The property of exact controllability for those type of systems is not fully understood yet.

System (1.1) can be seen as a toy model for such systems. Its study is an attempt to understand the phenomena governing the exact controllablity process.

Controllability of parabolic systems. The controllablity of parabolic systems has been intensively studied in the last decade (see for instance the review article [AKBGBdT11]). One of the challenging questions in this area is to understand the optimal geometric conditions on the control set $\omega$ and the coupling set $\mathcal{O}$, needed for null-controllability. The first positive result concerns the case where $\omega \cap \mathcal{O} \neq \emptyset$ (see [AKBGBdT11] or [Léa10]). As for the case $\omega \cap \mathcal{O}=\emptyset$, little is known. The idea of [ABL11, ABL12] was to make use of the transmutation method to reduce the parabolic problem to a system of coupled wave equations. This allowed to establish null-controllability of symmetric systems under the only condition that both $\omega$ and $\mathcal{O}$ satisfy GCC. In particular, this includes several situations where $\omega \cap \mathcal{O}=\emptyset$ (see [ABL11, ABL12] and the figures therein). However, in such results, $\omega$ and $\mathcal{O}$ both need to satisfy GCC, whereas for parabolic systems we expect a null controllability result to hold without any geometric assumptions on these two subsets. Concerning cascade heat equations, the only result (to our knowledge) is proved in one space dimension with the same strategy in [RdT11].

The results of the present work provide an extension of this result in general $n$-dimensional compact manifolds under geometric conditions.

Insensitizing controls for the wave equation. The question of insensitizing control for a wave equation, introduced by J.-L. Lions [Lio90] and addressed in [Dág06, Teb08] is the following. We consider the controlled wave equation

$$
\begin{cases}P u=b_{\omega}(x) f & \text { in }(0, T) \times \Omega, \\ \left.u\right|_{t=0}=u_{0}+\tau_{0} z_{0} & \text { in } \Omega, \\ \left.\partial_{t} u\right|_{t=0}=u_{1}+\tau_{1} z_{1} & \text { in } \Omega,\end{cases}
$$

where the data $\left(u_{0}, u_{1}\right) \in H^{1}(\Omega) \times L^{2}(\Omega)$ are fixed, and $\tau_{0} z_{0}, \tau_{1} z_{1}$ represent unknown noises, with

$$
\left\|z_{0}\right\|_{H^{1}(\Omega)}=\left\|z_{1}\right\|_{L^{2}(\Omega)}=1,
$$


and $\tau_{0}, \tau_{1} \in \mathbb{R}$. A control function $f \in L^{2}((0, T) \times \Omega)$ is said to insensitize the cost functional

$$
\Phi(u)=\frac{1}{2} \int_{0}^{T} \int_{\Omega} b(x)|u(t, x)|^{2} d x d t,
$$

if for any pair $\left(z_{0}, z_{1}\right)$ satisfying (1.3), the corresponding solution of (1.2) satisfies

$$
\left.\frac{d}{d \tau_{0}} \Phi(u)\right|_{\tau_{0}=\tau_{1}=0}=\left.\frac{d}{d \tau_{1}} \Phi(u)\right|_{\tau_{0}=\tau_{1}=0}=0
$$

This basically means that for this particular control function $f$, the cost functional (i.e. the local $L^{2}$ norm of the solution on $\mathcal{O}$ ) is insensitive to small variations of the initial data. This problem can be recast as a constrained coupled control problem of the form (1.1), to which our results will apply.

The main purposes of this article are to prove controllability for System (1.1), to find an explicit expression of the minimal control time in the simple situation where $\Omega$ is a compact manifold without boundary, and to describe precisely the microlocal properties of the optimal control operator, that is yielding the control function of minimal $L^{2}$-norm.

\subsection{Main results}

Our main results are threefold. First, we give a necessary and sufficient condition for the controllability of System (1.1). Second, we give a precise description of the optimal control operator associated to System (1.1). Third, we give sharp sufficient conditions for the controllability of similar systems, when the two waves propagate with different speeds.

\subsubsection{Controllability of System (1.1)}

To state our first main result, we introduce the adapted control time.

Definition 1.2. Given two sets $\omega$ and $\mathcal{O}$ both satisfying GCC, we set $T_{\omega \rightarrow \mathcal{O} \rightarrow \omega}$ to be the infimum of times $T>0$ for which the following assertion is satisfied:

every geodesic traveling at speed one in $\Omega$ meets $\omega$ in a time $t_{0}<T$, meets $\mathcal{O}$ in a time $t_{1} \in\left(t_{0}, T\right)$ and meets $\omega$ again in a time $t_{2} \in\left(t_{1}, T\right)$.

Note that in general $T_{\omega \rightarrow \mathcal{O} \rightarrow \omega} \neq T_{\mathcal{O} \rightarrow \omega \rightarrow \mathcal{O}}$, and that we have the estimate

$$
\max \left(T_{\mathcal{O}}, T_{\omega}\right) \leq T_{\omega \rightarrow \mathcal{O} \rightarrow \omega} \leq 2 T_{\omega}+T_{\mathcal{O}}
$$

We can now state our main controllability result (in the sense of Definition 1.1).

Theorem 1.3. Suppose that $b \geq 0$ on $\Omega$, and that both sets $\omega$ and $\mathcal{O}$ satisfy $G C C$. Then, System (1.1) is controllable if $T>T_{\omega \rightarrow \mathcal{O} \rightarrow \omega}$ and is not controllable if $T<T_{\omega \rightarrow \mathcal{O} \rightarrow \omega}$.

In particular this result holds without any assumption on the smallness of the coupling coefficient $b$ as is done in [ABL11, ABL12].

According to the Hilbert Uniqueness Method (HUM) of J.-L. Lions [Lio88] (detailed in Section 5.1 for the system we consider), the controllability property of Theorem 1.3 is equivalent to an observability inequality for the adjoint system. More precisely, System (1.1) is exactly controllable in time $T$ if and only if the inequality

$$
E_{-1}\left(v_{1}(0)\right)+E_{0}\left(v_{2}(0)\right) \leq C \int_{0}^{T} \int_{\omega}\left|b_{\omega} v_{2}\right|^{2} d x d t
$$

holds for every $\left(v_{1}, v_{2}\right) \in \mathscr{C}^{0}\left([0, T] ; H^{-1}(\Omega) \times L^{2}(\Omega)\right) \cap \mathscr{C}^{0}\left([0, T] ; H^{-2}(\Omega) \times H^{-1}(\Omega)\right)$ solutions of

$$
\begin{cases}P v_{1}=0 & \text { in }(0, T) \times \Omega, \\ P v_{2}=-b(x) v_{1} & \text { in }(0, T) \times \Omega .\end{cases}
$$


In the observability inequality (1.5), we use the notation

$$
E_{k}(v)=\|v\|_{H^{k}(\Omega)}^{2}+\left\|\partial_{t} v\right\|_{H^{k-1}(\Omega)}^{2}, \quad k \in \mathbb{Z}
$$

where the space $H^{s}(\Omega)$ is endowed with the norm

$$
\|v\|_{H^{s}(\Omega)}=\left\|(1-\Delta)^{\frac{s}{2}} v\right\|_{L^{2}(\Omega)}, \quad s \in \mathbb{R},
$$

and the associated inner product.

The proof of the observability inequality (1.5) is based on a contradiction argument, inspired by that of [Leb96]. Similarly, the key tools involved are microlocal defect measures introduced by P. Gérard [Gér91] and L. Tartar [Tar90], and used to solve control problems in [Leb96, Bur97a, BG97].

\subsubsection{Hilbert Uniqueness Method and description of the control}

An important feature of the Hilbert Uniqueness Method, as presented by Lions [Lio88], lays in the following two facts: the control one obtains, $f_{H U M}$ minimizes the cost functional $\|f\|_{L^{2}((0, T) \times \Omega)}^{2}$ among all $f \in L^{2}((0, T) \times \Omega)$ realizing a control for System (1.1) (see Section 5): it is the optimal $L^{2}$-control. Moreover, it is itself a solution of the adjoint system (for instance System (2.6) in our situation) for appropriate initial data, say $W^{0}$.

The Gramian operator $\mathcal{G}$ associated to Systems (1.1)-(1.6) is given by

$$
\int_{0}^{T} \int_{\omega}\left|b_{\omega} v_{2}\right|^{2} d x d t=(\mathcal{G} V, V)_{H^{-1}(\Omega) \times L^{2}(\Omega) \times H^{-2}(\Omega) \times H^{-1}(\Omega)},
$$

where $v_{2}$ is the solution of (1.6) associated to the initial data $\left.\left(v_{1}, v_{2}, \partial_{t} v_{1}, \partial_{t} v_{2}\right)\right|_{t=0}=V$. If the observability inequality (1.5) is satisfied, then, the HUM control operator is the inverse of the mapping $\mathcal{G}$. To the initial data $V$ to be controlled, the HUM operator maps the associated initial data $W^{0}$ for the adjoint system, giving rise to the control function $f_{H U M}$.

The second main goal of this article is to give an explicit representation of the HUM operator. We prove the following result (see Theorem 5.5 and Corollary 5.6).

1. The Gramian operator is a matrix of pseudodifferential operators of order zero. The determinant of its principal symbol takes essentially the following form

$$
\int_{0}^{T} \int_{0}^{T}\left(b_{\omega}^{2} \circ \varphi_{t_{1}}\right)\left(b_{\omega}^{2} \circ \varphi_{t_{2}}\right)\left(\int_{t_{1}}^{t_{2}} b \circ \varphi_{\sigma} d \sigma\right)^{2} d t_{1} d t_{2}
$$

where $\varphi_{\sigma}$ denotes the geodesic flow on $S^{*} \Omega$.

2. This operator is elliptic if and only if $T>T_{\omega \rightarrow \mathcal{O} \rightarrow \omega}$. This property provides a second proof of Theorem 1.3.

3. For $T>T_{\omega \rightarrow \mathcal{O} \rightarrow \omega}$, the HUM control operator is also a matrix of pseudodifferential operators of order zero.

A precise statement needs the introduction of some notation and will be given in Section 5.3. In particular, this result holds without any sign assumption on the function $b$. As a consequence, this method also provides a necessary and sufficient condition of (high-frequency) controllability for System 1.1 for any real-valued $b$, stated in Corollary 5.8.

The proof of this result is in the spirit of [DL09], and uses in an essential way the Egorov theorem. The information carried by microlocal defect measures is not sufficient to prove such a strong property of the HUM operator. Note that the third item above has several important consequences, as described in [DL09].

For the proof of these results we shall follow the program elaborated in [DL09] in the case of the wave equation. 


\subsubsection{The case of different speeds}

It appears also natural to consider the control problem for two coupled wave equations with different speeds:

$$
\begin{cases}P u_{1}+b(x) u_{2}=0 & \text { in }(0, T) \times \Omega, \\ P_{\gamma} u_{2}=b_{\omega}(x) f & \text { in }(0, T) \times \Omega,\end{cases}
$$

with $P=\partial_{t}^{2}-\Delta$ and $P_{\gamma}=\partial_{t}^{2}-\gamma^{2} \Delta$ for some $\gamma>0, \gamma \neq 1$. In this case, we shall say that System (1.7) is controllable in the space $H^{s+1} \times H^{s}$ in time $T>0$ if for any target $\left(\tilde{u}_{1}^{0}, \tilde{u}_{2}^{0}, \tilde{u}_{1}^{1}, \tilde{u}_{2}^{1}\right) \in$ $H^{s+1}(\Omega) \times H^{1}(\Omega) \times H^{s}(\Omega) \times L^{2}(\Omega)$, there exists a control function $f \in L^{2}((0, T) \times \Omega)$ such that the solution of (1.7) starting from rest $\left.\left(u_{1}, u_{2}, \partial_{t} u_{1}, \partial_{t} u_{2}\right)\right|_{t=0}=(0,0,0,0)$ satisfies $\left.\left(u_{1}, u_{2}, \partial_{t} u_{1}, \partial_{t} u_{2}\right)\right|_{t=T}=$ $\left(\tilde{u}_{1}^{0}, \tilde{u}_{2}^{0}, \tilde{u}_{1}^{1}, \tilde{u}_{2}^{1}\right)$. This naturally requires the system to be well-posed in these spaces. Then, this notion is also equivalent to exact and null-controllability.

Definition 1.4. For a subset $U \subset \Omega$ satisfying GCC and $\gamma>0$, we define $T_{U}(\gamma)$ to be the infimum of times $T$ such that every geodesic traveling at speed $\gamma$ in $\Omega$ meets $U$ in a time $t<T$.

In particular, with the notation above, we have $T_{U}=T_{U}(1)$ the usual GCC time of the subset $U$.

With this definition, we have the following result.

Theorem 1.5. 1. For any $s<2$ any $T>0$, and any open sets $\omega$ and $\mathcal{O}$, System (1.7) is not controllable in $H^{s+1} \times H^{s}$ in time $T$.

2. Suppose that $\bar{\omega} \cap \overline{\mathcal{O}}$ does not satisfy GCC. Then for any $s \in \mathbb{R}$ and any $T>0$, System (1.7) is not controllable in $H^{s+1} \times H^{s}$ in time $T$.

3. Suppose that $\omega \cap \mathcal{O}$ satisfies GCC. Then, System (1.7) is controllable in $H^{3} \times H^{2}$ for $T>$ $\max \left\{T_{\omega \cap \mathcal{O}}(1), T_{\omega}(\gamma)\right\}$ and is not controllable for $T<\max \left\{T_{\bar{\omega} \cap \overline{\mathcal{O}}}(1), T_{\omega}(\gamma)\right\}$.

Remark 1.6. An extension of these results should be possible in the case of different Riemannian metrics yielding (partially or totally) non-intersecting characteristic sets of the two wave operators.

In some sense, our results show that the most interesting problem concerns the case where the two waves propagate with the same speed.

\subsection{Comments and outline}

\subsubsection{Regarding the time $T_{\omega \rightarrow \mathcal{O} \rightarrow \omega}$}

The time $T_{\omega \rightarrow \mathcal{O} \rightarrow \omega}$ might be surprising at first sight. It can be interpreted in the following way: to be able to detect the energy of both components of System (1.6) from the observation on $\omega$ of the second one only, the polarization of the state along each ray of geometric optics has to change its direction between two passages in the control region $\omega$. This change of polarization arises only when this ray enters the coupling set $\mathcal{O}$.

A description of the notion of polarization, as well as an insight on this geometrical interpretation may be found in [BL01].

A comparable geometric condition already appears in the study of the decay rates for the thermoelasticity system, see [LZ99] and [BL01].

\subsubsection{Comparing the different methods of proofs of Theorem $\mathbf{1 . 3}$}

In the case of a scalar wave equation, there exist, to our knowledge, three different methods for proving the (high-frequency) observability on a compact manifold, with optimal conditions on the geometry and the control time. The first one, introduced by Rauch and Taylor [RT74], and further developped by Bardos, Lebeau and Rauch [BLR92] deals with the wavefront sets propagation and uses in a crucial way the Hörmander theorem on propagation of singularities.

The second method, introduced by Lebeau [Leb96], further used by Burq [Bur97a], Burq and Gérard [BG97] is based on microlocal defect measures and the propagation of their support. 
The last method relies on the use of the Egorov theorem (i.e., the theory of Fourier integral operators) and was recently proposed by Dehman and Lebeau [DL09]. Note on the one hand that the first two methods also apply (with considerable additional difficulties) in the case of a manifold with boundary. On the other hand, there is no analogue of the Egorov theorem in such case, and the last method fails to apply. However, in [DL09], the authors show that the FIO Egorov approach provides additional insight on the control problem. In particular, they prove that the HUM operator (the optimal control operator) is (essentially) a pseudodifferential operator and they exhibit its principal symbol.

Here, we provide two different proofs of Theorem 1.3. The first one (using microlocal defect measures) has the advantage of working with limited smoothness (we basically only have to assume that $b_{\omega} \in \mathscr{C}^{0}(\Omega)$ and $b \in W^{1, \infty}(\Omega)$ ). Moreover, this method could be extended to boundary value problems.

The second proof, using the Egorov theorem has the advantage of working as well with coupling functions $b$ changing signs. Moreover, this method not only provides the observability inequality, but also several additional informations on the microlocal nature of the HUM control operator.

Note finally that a proof based on wavefront sets might be possible, with the use of the polarization wavefront set of Dencker [Den82].

\subsubsection{The case of an open domain $\Omega \subset \mathbb{R}^{n}$}

Naturally, the same problem can also be adressed on a bounded smooth open set $\Omega \subset \mathbb{R}^{n}$ (or a manifold with boundary), with (for instance) Dirichlet conditions on the boundary. The method of proof using microlocal defect measures may also work in this setting. However, one of its key points is a propagation result of the microlocal defect measures (analogous of Lemma 3.3 of the present article) up to the boundary (see [Leb96, Bur97a, BG97] for scalar equations and [BL01] for systems). This technical point needs more care, and is the goal of an ongoing work.

\subsubsection{Application to parabolic systems}

The "transmutation strategy" used in [ABL11, ABL12] can also be followed here. As a corollary of Theorem 1.3, it furnishes several null-controllability results for cascade parabolic (or Schrödinger) systems (for all positive time), in cases where the control region $\omega$ and the coupling region $\mathcal{O}$ do not intersect. However, in such results, $\omega$ and $\mathcal{O}$ have to satisfy GCC, whereas for parabolic systems we expect a null-controllability result to hold without any geometric assumptions on these two subsets. Note that a similar result has been recently obtained in [AB12], with a completely different method.

\subsubsection{Application to insensitizing controls}

We first recall that the problem of insensitizing controls is equivalent (see [Dág06] or [Teb08]) to the fact that the observability inequality

$$
E_{-1}\left(v_{1}(0)\right) \leq C \int_{0}^{T} \int_{\omega}\left|b_{\omega} v_{2}\right|^{2} d x d t
$$

holds for every $\left(v_{1}, v_{2}\right) \in \mathscr{C}^{0}\left([0, T] ; H^{-1}(\Omega) \times L^{2}(\Omega)\right) \cap \mathscr{C}^{0}\left([0, T] ; H^{-2}(\Omega) \times H^{-1}(\Omega)\right)$ solutions of

$$
\begin{cases}P v_{1}=0 & \text { in }(0, T) \times \Omega, \\ P v_{2}=b(x) v_{1} & \text { in }(0, T) \times \Omega, \\ \left.\left(v_{2}, \partial_{t} v_{2}\right)\right|_{t=T}=(0,0) & \text { in } \Omega .\end{cases}
$$

Since Theorem 1.3 also holds for $b \leq 0, \mathcal{O}=\{b<0\}$ (changing $v_{1}$ in $-v_{1}$ ), we directly obtain the following result.

Corollary 1.7. Suppose that both $\omega$ and $\mathcal{O}$ satisfy $G C C$, and that $T>T_{\omega \rightarrow \mathcal{O} \rightarrow \omega}$. Then for all $\left(u_{0}, u_{1}\right) \in H^{1}(\Omega) \times L^{2}(\Omega)$, there exist a control function for System (1.2) that insensitizes the functional $\Phi$ defined in (1.4). 
Since GCC is necessary for both sets $\omega$ and $\mathcal{O}$, the geometric conditions obtained here are optimal. Note that the only known results to our knowledge are the one dimensional case, see [Dág06], and the case where $\mathcal{O} \cap \omega$ satisfies the multiplier condition of Lions, see [Teb08].

\subsubsection{Outline.}

The outline of this article is the following. In Section 2, we give some notation, define the tools used in the main part of the article and recall some basic well-posedness results.

In Section 3, we prove that the observability inequality holds if $T>T_{\omega \rightarrow \mathcal{O} \rightarrow \omega}$. Conversely, we prove in Section 4 that the observability inequality does not hold in the case $T<T_{\omega \rightarrow \mathcal{O} \rightarrow \omega}$.

In Section 5, we develop the Hilbert Uniqueness Method. We first prove the equivalence between controllability and observability in Section 5.1 and we define the HUM control operator in Section 5.2. Then, we give the explicit characterization of the HUM operator in Section 5.3.

Finally, in Section 6, we provide proofs for the positive and negative results concerning the case of coupled waves with different speeds.

\section{Preliminary remarks, definitions and notation}

We define the manifold $M=\mathbb{R} \times \Omega$ and its restriction to $(0, T): M_{T}=(0, T) \times \Omega=\{(t, x) \in$ $M$ such that $t \in(0, T)\}$. We also write $T^{*} M_{T}$ the restriction of the cotangent bundle of $M$ to $(0, T)$, i.e. $T^{*} M_{T}=\left\{(t, x, \tau, \eta) \in T^{*} M, t \in(0, T)\right\}$. Setting $|\eta|_{x}^{2}=g_{x}(\eta, \eta)$ the Riemannian norm in the cotangent space of $\Omega$ at $x$, we define

$$
S^{*} M=\left\{(t, x, \tau, \eta) \in T^{*} M,|\tau|^{2}+|\eta|_{x}^{2}=1\right\},
$$

the cosphere bundle of $M$, and similarly $S^{*} M_{T}$ its restriction to $(0, T)$. We denote by $\pi: S^{*} M \rightarrow M$ the natural projection, which also maps $S^{*} M_{T}$ onto $M_{T}$. We shall also use the associated cosphere bundle in the spatial avariables only,

$$
S^{*} \Omega=\left\{(x, \eta) \in T^{*} \Omega,|\eta|_{x}^{2}=1 / 2\right\} .
$$

\subsection{Symbols, operators and measures on the cosphere bundle}

Here, we follow [Bur97b, Section 1.1] for the notation. We denote by $H^{k}\left(X ; \mathbb{C}^{j}\right)$ or $H_{\mathrm{loc}}^{k}\left(X ; \mathbb{C}^{j}\right)$, with $j=1$ or 2 and $X=\Omega, M$, or $M_{T}$, the usual Sobolev space for functions valued in $\mathbb{C}^{j}$, endowed with the natural inner product and norm. In particular, the $L^{2}\left(X ; \mathbb{C}^{j}\right)$ inner product is denoted by $(\cdot, \cdot)_{L^{2}\left(X ; \mathbb{C}^{j}\right)} \cdot$

We define $S_{\mathrm{phg}}^{m}\left(T^{*} M_{T} ; \mathbb{C}^{j \times j}\right)$, with $j=1$ or 2 as the set of matrix valued polyhomogeneous symbols of order $m$ on $M_{T}$ with compact support in $M_{T}$. We recall that symbols in the class $S_{\mathrm{phg}}^{m}\left(T^{*} \mathbb{R}^{n} ; \mathbb{C}^{j \times j}\right)$ behave well with respect to changes of variables, up to symbols in $S_{\mathrm{phg}}^{m-1}\left(T^{*} \mathbb{R}^{n} ; \mathbb{C}^{j \times j}\right)$ (see [Hör85, Theorem 18.1.17 and Lemma 18.1.18]).

For any $m$, the restriction to the sphere

$$
S_{\mathrm{phg}}^{m}\left(T^{*} M_{T} ; \mathbb{C}^{j \times j}\right) \rightarrow \mathscr{C}_{c}^{\infty}\left(S^{*} M_{T} ; \mathbb{C}^{j \times j}\right),\left.\quad a \rightarrow a\right|_{S^{*} M_{T}},
$$

is onto. This will allow us to identify an homogeneous symbol with a smooth function on the sphere.

We denote by $\Psi_{\mathrm{phg}}^{m}\left(M_{T} ; \mathbb{C}^{j \times j}\right)$, with $j=1$ or 2 the space of polyhomogeneous pseudodifferential operators of order $m$ on $M_{T}$, with a compactly supported kernel in $M_{T} \times M_{T}$ : one says that $A \in$ $\Psi_{\mathrm{phg}}^{m}\left(M_{T} ; \mathbb{C}\right)$ if

1. its kernel $K(x, y) \in \mathscr{D}^{\prime}\left(M_{T} \times M_{T}\right)$ is such that $\operatorname{supp}(K)$ is compact in $M_{T}$;

2. $K(x, y)$ is smooth away from the diagonal $\Delta_{M_{T}}=\left\{(t, x ; t, x) ;(t, x) \in M_{T}\right\}$; 
3. for every coordinate patch $M_{T, \kappa} \subset M_{T}$ with coordinates $M_{T, \kappa} \ni(t, x) \mapsto \kappa(t, x) \in \tilde{M}_{T, \kappa} \subset$ $\mathbb{R}^{n+1}$ and all $\phi_{0}, \phi_{1} \in \mathscr{C}_{c}^{\infty}\left(\tilde{M}_{T, \kappa}\right)$ the map

$$
u \mapsto \phi_{1}\left(\kappa^{-1}\right)^{*} A \kappa^{*}\left(\phi_{0} u\right)
$$

is in $\mathrm{Op}\left(S_{\mathrm{phg}}^{m}\left(\mathbb{R}^{n+1} \times \mathbb{R}^{n+1}\right)\right)$.

For $A \in \Psi_{\mathrm{phg}}^{m}\left(M_{T} ; \mathbb{C}^{j \times j}\right)$, we denote by $\sigma_{m}(A) \in S_{\mathrm{phg}}^{m}\left(T^{*} M_{T} ; \mathbb{C}^{j \times j}\right)$ the principal symbol of $A$ (see [Hör85, Chapter 18.1]). Note that the principal symbol is uniquely defined in $S_{\mathrm{phg}}^{m}\left(T^{*} M_{T} ; \mathbb{C}^{j \times j}\right)$ because of the polyhomogeneous structure (see the remark following Definition 18.1.20 in [Hör85]). The application $\sigma_{m}$ enjoys the following properties

- $\sigma_{m}: \Psi_{\mathrm{phg}}^{m}\left(M_{T} ; \mathbb{C}^{j \times j}\right) \rightarrow S_{\mathrm{phg}}^{m}\left(T^{*} M_{T} ; \mathbb{C}^{j \times j}\right)$ is onto.

- For all $A \in \Psi_{\mathrm{phg}}^{m}\left(M_{T} ; \mathbb{C}^{j \times j}\right), \sigma_{m}(A)=0$ if and only if $A \in \Psi_{\mathrm{phg}}^{m-1}\left(M_{T} ; \mathbb{C}^{j \times j}\right)$.

- For all $A \in \Psi_{\mathrm{phg}}^{m}\left(M_{T} ; \mathbb{C}^{j \times j}\right), \sigma_{m}\left(A^{*}\right)={ }^{t} \overline{\sigma_{m}(A)}$.

- For all $A_{1} \in \Psi_{\mathrm{phg}}^{m_{1}}\left(M_{T} ; \mathbb{C}^{j \times j}\right)$ and $A_{2} \in \Psi_{\mathrm{phg}}^{m_{2}}\left(M_{T} ; \mathbb{C}^{j \times j}\right)$, we have $A_{1} A_{2} \in \Psi_{\mathrm{phg}}^{m_{1}+m_{2}}\left(M_{T} ; \mathbb{C}^{j \times j}\right)$ with

$$
\sigma_{m_{1}+m_{2}}\left(A_{1} A_{2}\right)=\sigma_{m_{1}}\left(A_{1}\right) \sigma_{m_{2}}\left(A_{2}\right) .
$$

- For all $A_{1} \in \Psi_{\mathrm{phg}}^{m_{1}}\left(M_{T} ; \mathbb{C}\right)$ and $A_{2} \in \Psi_{\mathrm{phg}}^{m_{2}}\left(M_{T} ; \mathbb{C}\right)$, we have $\left[A_{1}, A_{2}\right]=A_{1} A_{2}-A_{2} A_{1} \in$ $\Psi_{\mathrm{phg}}^{m_{1}+m_{2}-1}\left(M_{T} ; \mathbb{C}\right)$ with

$$
\sigma_{m_{1}+m_{2}-1}\left(\left[A_{1}, A_{2}\right]\right)=\frac{1}{i}\left\{\sigma_{m_{1}}\left(A_{1}\right), \sigma_{m_{2}}\left(A_{2}\right)\right\} .
$$

Here, $\left\{a_{1}, a_{2}\right\}$ denotes the Poisson bracket, given in local charts by

$$
\left\{a_{1}, a_{2}\right\}=\partial_{\tau} a_{1} \partial_{t} a_{2}-\partial_{t} a_{1} \partial_{\tau} a_{2}+\sum_{l}\left(\partial_{\xi_{l}} a_{1} \partial_{x_{l}} a_{2}-\partial_{x_{l}} a_{1} \partial_{\xi_{l}} a_{2}\right)
$$

- If $A \in \Psi_{\mathrm{phg}}^{m}\left(M_{T} ; \mathbb{C}^{j \times j}\right)$, then $A$ maps continuously $H^{k}\left(M_{T} ; \mathbb{C}^{j}\right)$ into $H^{k-m}\left(M_{T} ; \mathbb{C}^{j}\right)$ (resp. $H_{\mathrm{loc}}^{k}\left(M_{T} ; \mathbb{C}^{j}\right)$ into $\left.H_{\mathrm{loc}}^{k-m}\left(M_{T} ; \mathbb{C}^{j}\right)\right)$. In particular, for $m<0, A$ is compact on $L^{2}\left(M_{T} ; \mathbb{C}^{j}\right)$.

Given an operator $A \in \Psi_{\mathrm{phg}}^{m}\left(M_{T} ; \mathbb{C}\right)$, we define $\operatorname{Char}(A)=\left\{\rho \in T^{*} M, \sigma_{m}(A)(\rho)=0\right\}$.

At places we shall need to consider pseudodifferential operators acting on $\Omega$ yet depending upon the parameter $t \in(0, T)$ with some smoothness with respect to $t$. Let $k \in \mathbb{N} \cup\{\infty\}$, we say that $A_{t} \in \mathscr{C}^{k}\left((0, T), \mathrm{Op}\left(S_{\mathrm{phg}}^{m}\left(\mathbb{R}^{n} \times \mathbb{R}^{n}\right)\right)\right)$ if $A_{t}=\mathrm{Op}\left(a_{t}\right)$ with $a_{t} \in \mathscr{C}^{k}\left((0, T), S_{\mathrm{phg}}^{m}\left(\mathbb{R}^{n} \times \mathbb{R}^{n}\right)\right)$. Next we say that $A_{t} \in \mathscr{C}^{k}\left((0, T), \Psi_{\mathrm{phg}}^{m}(\Omega)\right)$ if

1. its kernel $K_{t}(x, y)$ is in $\mathscr{C}^{k}\left((0, T), \Omega \times \Omega \backslash \Delta_{\Omega}\right)$ where $\Delta_{\Omega}=\{(x, x) ; x \in \Omega\}$;

2. for every coordinate patch $\Omega_{\kappa} \subset \Omega$ with coordinates $\Omega_{\kappa} \ni x \mapsto \kappa(x) \in \tilde{\Omega}_{\kappa} \subset \mathbb{R}^{n}$ and all $\phi_{0}$, $\phi_{1} \in \mathscr{C}_{c}^{\infty}\left(\tilde{\Omega}_{\kappa}\right)$ the map

$$
u \mapsto \phi_{1}\left(\kappa^{-1}\right)^{*} A_{t} \kappa^{*}\left(\phi_{0} u\right)
$$

is in $\mathscr{C}^{k}\left((0, T), \operatorname{Op}\left(S_{\mathrm{phg}}^{m}\left(\mathbb{R}^{n} \times \mathbb{R}^{n}\right)\right)\right)$.

In particular we shall use the following form of the Egorov theorem.

Theorem 2.1. Let $A_{t} \in \mathscr{C}^{\infty}\left((0, T), \Psi_{\mathrm{phg}}^{1}(\Omega)\right)$ with real principal symbol $a_{1, t}$ and $P \in \Psi_{\mathrm{phg}}^{m}(\Omega)$, $m \in \mathbb{R}$. Define $S\left(s^{\prime}, s\right)$ as the solution operator for the Cauchy problem

$$
\partial_{t} u+i A_{t} u=0,\left.\quad u\right|_{t=s}=u_{0}
$$


i.e., $u\left(s^{\prime}\right)=S\left(s^{\prime}, s\right) u_{0}$. Then there exists $Q_{t} \in \mathscr{C}^{\infty}\left((0, T), \Psi^{m}(\Omega)\right)$ such that, for all $\sigma, N \in \mathbb{R}$, we have

$$
S(t, 0) P S(0, t)-Q_{t} \in \mathscr{C}^{\infty}\left((0, T), \mathcal{L}\left(H^{\sigma}(\Omega), H^{\sigma+N}(\Omega)\right)\right)
$$

and the principal symbol of $Q_{t}$ is given by $q_{t} \in \mathscr{C}^{\infty}\left((0, T), S_{\mathrm{phg}}^{1}\left(T^{*} \Omega\right)\right)$ with $q_{t}=p \circ \chi_{0, t}$ where $\rho(s, t)=\chi_{s, t}\left(\rho_{0}\right)$ is given by the flow of the Hamiltonian vector field associated with $a_{1, t}$ :

$$
\frac{d}{d s} \rho(s, t)=H_{a_{1, t}}(\rho(s, t)), \quad \rho(t, t)=\rho_{0}
$$

The proof can be adapted for instance from that given in [Tay91, Theorem 0.9.A]. The notion of smoothing operators appearing in the statement of the above theorem is precised in following definition.

Definition 2.2 (Smoothing operators). Let $A: \mathscr{D}^{\prime}(\Omega) \rightarrow \mathscr{D}^{\prime}(\Omega)$ be a linear operator and $k>0$. We say that $A$ is $k$-smoothing if $A \in \mathcal{L}\left(H^{s}(\Omega) ; H^{s+k}(\Omega)\right)$ for all $s \in \mathbb{R}$. We say that $A$ is infinitely smoothing if $A$ is $k$-smoothing for all $k>0$.

Moreover, we say that $A \in \mathcal{R}^{k}(\Omega)$ if $A \in \mathcal{L}\left(H^{s}(\Omega): H^{s+k}\right)$ for all $s \geq 0$. We set $\mathcal{R}^{\infty}(\Omega)=$ $\bigcap_{k>0} \mathcal{R}^{k}(\Omega)$.

Note in particular that $k$-smoothing operators are in $\mathcal{R}^{k}(\Omega)$. Moreover, for $k>0$, operators in $\Psi_{\text {phg }}^{-k}(\Omega)$ are $k$-smoothing.

We recall that $-\Delta$ denotes the Laplace operator on $\Omega$, and that we have

$$
-\Delta \in \Psi_{\mathrm{phg}}^{2}(\Omega), \quad \text { with } \quad \sigma_{2}(-\Delta)(x, \eta)=|\eta|_{x}^{2}
$$

It will also be useful to define a function $\tilde{\lambda} \in \mathscr{C}^{\infty}\left(T^{*} M\right)$ such that

$$
\begin{aligned}
& \tilde{\lambda}(t, x, \tau, \eta)=\left(|\tau|^{2}+|\eta|_{x}^{2}\right)^{\frac{1}{2}} \quad \text { for }(t, x, \tau, \eta) \in T^{*} M, \text { with }\left(|\tau|^{2}+|\eta|_{x}^{2}\right)^{\frac{1}{2}} \geq \frac{1}{2} \\
& \tilde{\lambda}(t, x, \tau, \eta) \geq C>0 \quad \text { for }(t, x, \tau, \eta) \in T^{*} M, \text { with }\left(|\tau|^{2}+|\eta|_{x}^{2}\right)^{\frac{1}{2}} \leq \frac{1}{2} .
\end{aligned}
$$

This gives $\chi \tilde{\lambda}^{m} \in S_{\mathrm{phg}}^{m}\left(T^{*} M ; \mathbb{C}\right)$ if $m \in \mathbb{Z}$ and $\chi \in \mathscr{C}_{c}^{\infty}(M)$.

Finally, we define $\mathcal{M}\left(S^{*} M_{T} ; \mathbb{R}\right)$ to be the set of real valued measures on $S^{*} M_{T}, \mathcal{M}_{+}\left(S^{*} M_{T}\right)$ the set of positive measures on $S^{*} M_{T}$, and $\mathcal{M}_{+}\left(S^{*} M_{T} ; \mathbb{C}^{2 \times 2}\right)$ the set of measures with values in non-negative hermitian $2 \times 2$ matrices. For $\mu \in \mathcal{M}\left(S^{*} M_{T} ; \mathbb{R}\right)\left(\right.$ resp. $\left.\mu \in \mathcal{M}_{+}\left(S^{*} M_{T} ; \mathbb{C}^{2 \times 2}\right)\right)$ and $a \in \mathscr{C}_{c}^{0}\left(S^{*} M_{T} ; \mathbb{R}\right)\left(\right.$ resp. $\left.a \in \mathscr{C}_{c}^{0}\left(S^{*} M_{T} ; \mathbb{C}^{2 \times 2}\right)\right)$, we shall write

$$
\langle\mu, a\rangle_{S^{*} M_{T}}=\int_{S^{*} M_{T}} a(\rho) \mu(d \rho), \quad\left(\operatorname{resp} .\langle\mu, a\rangle_{S^{*} M_{T}}=\int_{S^{*} M_{T}} \operatorname{tr}\{a(\rho) \mu(d \rho)\}\right)
$$

for the duality bracket. The same notation will also be used for $a \in S_{\mathrm{phg}}^{0}\left(T^{*} M_{T} ; \mathbb{R}\right)$ (resp. $a \in$ $\left.S_{\mathrm{phg}}^{0}\left(T^{*} M_{T} ; \mathbb{C}^{2 \times 2}\right)\right)$ according to the identification map (2.1).

Observe that the Laplace operator is not coercive since $-\Delta(1)=0$. This can be cumbersome at places. As a remedy, we introduce more convenient spaces and scalar product. Let $\left(e_{j}\right)_{j \in \mathbb{N}}$ be a Hilbert basis of eigenfunctions of $-\Delta$, associated to the eigenvalues $\left(\kappa_{j}\right)_{j \in \mathbb{N}}$. In particular, we have $\kappa_{0}=0$ and $e_{0}=1 / \sqrt{|\Omega|}$. Following the notation of [DL09], we set

$$
L_{+}^{2}(\Omega):=\left\{\sum_{j \geq 1} a_{j} e_{j},\left(a_{j}\right) \in \ell^{2}\right\}=\left\{f \in L^{2}(\Omega), \int_{\Omega} f(x) d x=0\right\}=\Pi_{+} L^{2}(\Omega),
$$

with

$$
\Pi_{0} f=\left(\frac{1}{\sqrt{|\Omega|}} \int_{\Omega} f(x) d x\right) e_{0}=\left(f, e_{0}\right)_{L^{2}(\Omega)} e_{0}, \quad \text { and } \quad \Pi_{+} f=f-\Pi_{0} f
$$


Note that we have

$$
\Pi_{0} \in \Psi_{\mathrm{phg}}^{-\infty}(\Omega)
$$

since $\Pi_{0}$ maps $\mathscr{D}^{\prime}(\Omega)$ into $\mathscr{C}^{\infty}(\Omega)$, i.e. has a $\mathscr{C}^{\infty}$ kernel (this is true in fact in a more general setting of functional calculus, see [Tay81, Chapter 12]). Hence

$$
\Pi_{+}=\mathrm{Id}-\Pi_{0} \in \Psi_{\mathrm{phg}}^{0}(\Omega), \quad \text { with } \quad \sigma_{0}\left(\Pi_{+}\right)=1 .
$$

We also define $H_{+}^{s}(\Omega)=\Pi_{+} H^{s}(\Omega)$ for $s \in \mathbb{R}$, and in particular $H_{+}^{s}(\Omega)=H^{s}(\Omega) \cap L_{+}^{2}(\Omega)$ if $s \geq 0$.

We shall often use the selfadjoint operator $\lambda=\sqrt{-\Delta}$, classically defined by

$$
\lambda f=\sum_{j \in \mathbb{N}} \sqrt{\kappa_{j}}\left(f, e_{j}\right)_{L^{2}(\Omega)} e_{j}, \quad D(\lambda)=H^{1}(\Omega) .
$$

In particular, we have $\lambda e_{0}=0$ and $\lambda$ is an isomorphism from $H_{+}^{s+1}(\Omega)$ onto $H_{+}^{s}(\Omega)$. We shall denote by $\lambda^{-1} \in \mathcal{L}\left(H_{+}^{s}(\Omega) ; H_{+}^{s+1}(\Omega)\right)$ its inverse. Moreover, according to [See67] (or [Shu01, Theorem 11.2]), we have

$$
\lambda \in \Psi_{\text {phg }}^{1}(\Omega), \quad \text { with } \quad \sigma_{1}(\lambda)(x, \eta)=|\eta|_{x}, \quad(x, \eta) \in T^{*} \Omega \backslash 0 .
$$

We denote by $\left(e^{i t \lambda}\right)_{t \in \mathbb{R}}$ the group on $H^{s}(\Omega)$ generated by $i \lambda$. Note that $e^{i t \lambda}$ preserves the spaces $H_{+}^{s}(\Omega)$.

The decomposition (splitting) of the operator $P$ into $P=-L_{+} L_{-}$, with

$$
L_{+}=\frac{1}{i} \partial_{t}-\lambda \quad \text { and } \quad L_{-}=\frac{1}{i} \partial_{t}+\lambda,
$$

will also be useful in the following. Even though $L_{ \pm}$is not a pseudodifferential operator on $M^{1}$, we shall write

$$
\ell_{+}=\sigma_{1}\left(L_{+}\right)=\tau-|\eta|_{x}, \quad \ell_{-}=\sigma_{1}\left(L_{-}\right)=\tau+|\eta|_{x},
$$

and refer to these functions as "the principal symbol of $L_{+}$and $L_{-}$".

\subsection{Some geometric facts}

In local coordinates, we write $g_{i j}$ for the metric $g$ on the tangent bundle $T M$. As a metric on the cotangent bundle $T^{*} M, g$ is given by $g^{i j}$ in local coordinates.

The principal symbol of the operator $P\left(t, x, \partial_{t}, \partial_{x}\right)$ is given by

$$
\sigma_{2}(P)(t, x, \tau, \eta)=p(t, x, \tau, \eta)=-\tau^{2}+|\eta|_{x}^{2}, \quad \text { for }(t, x, \tau, \eta) \in \mathbb{R} \times \Omega \times \mathbb{R} \times T_{x}^{*} \Omega \subset T^{*} M .
$$

We denote by $H_{p}$ the associated Hamiltonian vector field. In local coordinates, we have

$$
p=-|\tau|^{2}+\sum_{i, j} g^{i j} \eta_{i} \eta_{j} \quad \text { and } \quad H_{p}=\left(\nabla_{\tau, \eta} p,-\nabla_{t, x} p\right) .
$$

Note that for $a \in S_{\mathrm{phg}}^{m}\left(T^{*} M ; \mathbb{C}\right)$, we have $H_{p} a=\{p, a\}$. We shall make use of the Hamiltonian flow map $\phi_{s}$, i.e. the maximal solutions of

$$
\frac{d}{d s} \phi_{s}(\rho)=H_{p}\left(\phi_{s}(\rho)\right), \quad \phi_{0}(\rho)=\rho \in T^{*} M \backslash 0 .
$$

Let $\Gamma$ be an integral curve of (2.4). First notice that $p$ is constant along $\Gamma$ since $H_{p} p=0$. In particular, the flow $\phi_{s}$ preserves Char $(P)$. Moreover, as $g$ is independant of time $t,(2.4)$ also gives $\partial_{t} p=0$. Writing $\phi_{s}(\rho)=(t(s), x(s), \tau(s), \eta(s))$, this implies that $\tau$ is constant along $\Gamma$. As is done classically, we call bicharacteristics the integral curves for which $p=0$. Then $|\eta|_{x}^{2}=|\tau|^{2}$ is also constant along bicharacteristics. Observe then that (2.4) defines a flow on the manifold

$$
\operatorname{Char}(P) \cap S^{*} M=\left\{(t, x, \tau, \xi),|\tau|^{2}=1 / 2 \text { and }|\eta|_{x}^{2}=1 / 2\right\} .
$$

Now, we can rewrite the geometric condition given in Definition 1.2 in terms of bicharacteristics of the operator $P$.

\footnotetext{
${ }^{1}$ Observe that $\ell_{+}$and $\ell_{-}$do not satisfy the proper estimate in the cone $|\tau| \geq C|\eta|_{x}$.
} 
Definition 2.3. The time $T_{\omega \rightarrow \mathcal{O} \rightarrow \omega}$ is the infimum of times $T>0$ for which the following assertion is satisfied:

for any $\rho_{*}=\left(0, x_{*}, \tau_{*}, \eta_{*}\right) \in \operatorname{Char}(P) \cap S^{*} M$, there exists $0<t_{0}<t_{1}<t_{2}<T$ such that we have $\pi\left(\phi_{t_{0}}\left(\rho_{*}\right)\right) \in(0, T) \times \omega, \quad \pi\left(\phi_{t_{1}}\left(\rho_{*}\right)\right) \in(0, T) \times \mathcal{O}, \quad \pi\left(\phi_{t_{2}}\left(\rho_{*}\right)\right) \in(0, T) \times \omega$.

We remark that $\operatorname{Char}(P) \cap S^{*} M$ has two connected components given by

$$
\begin{aligned}
\operatorname{Char}(P) \cap S^{*} M & =\left(\operatorname{Char}\left(L^{+}\right) \cap S^{*} M\right) \cup\left(\operatorname{Char}\left(L^{-}\right) \cap S^{*} M\right) \\
& =\left\{\tau=1 / \sqrt{2} \text { and }|\eta|_{x}=1 / \sqrt{2}\right\} \cup\left\{\tau=-1 / \sqrt{2} \text { and }|\eta|_{x}=1 / \sqrt{2}\right\} .
\end{aligned}
$$

We shall denote by $\phi_{s}^{ \pm}$the bicharacteristic flow associated with $\ell_{ \pm}$, i.e., the maximal solutions of

$$
\frac{d}{d s} \phi_{s}^{ \pm}(\rho)=H_{\ell_{ \pm}}\left(\phi_{s}^{ \pm}(\rho)\right), \quad \phi_{0}^{ \pm}(\rho)=\rho \in T^{*} M \backslash 0 .
$$

As we have $\partial_{t} \ell_{ \pm}=0$ and $\partial_{\tau} \ell_{ \pm}=1$, the flow $\phi_{s}^{ \pm}$can be written under the form

$$
\phi_{s}^{ \pm}(t, \tau, x, \eta)=\left(t+s, \tau, \varphi_{s}^{ \pm}(x, \eta)\right),
$$

where

$$
\frac{d}{d s} \varphi_{s}^{ \pm}(x, \eta)=H_{\mp|\eta|_{x}}\left(\varphi_{s}^{ \pm}(x, \eta)\right), \quad \varphi_{0}^{ \pm}(x, \eta)=(x, \eta) \in T^{*} \Omega \backslash 0 .
$$

Note that $\varphi_{s}^{ \pm}$is the Hamiltonian flow associated with the operator $\mp \lambda$. In particular, notice that one has $\varphi_{-s}^{-}(x, \eta)=\varphi_{s}^{+}(x, \eta)$. With this notation, we define now the adapted minimal time for waves with positive/negative frequencies, $T_{\omega \rightarrow \mathcal{O} \rightarrow \omega}^{ \pm}$, and we provide a new definition of the minimal control time $T_{\omega \rightarrow \mathcal{O} \rightarrow \omega}$.

Definition 2.4. The time $T_{\omega \rightarrow \mathcal{O} \rightarrow \omega}^{ \pm}$is the infimum of times $T>0$ for which the following assertion is satisfied:

$$
\begin{aligned}
& \text { for any }(x, \eta) \in S^{*} \Omega \text {, there exists } 0<t_{0}<t_{1}<t_{2}<T \text { such that we have } \\
& b_{\omega} \circ \tilde{\pi} \circ \varphi_{t_{0}}^{ \pm}(x, \eta) \neq 0, \quad b \circ \tilde{\pi} \circ \varphi_{t_{1}}^{ \pm}(x, \eta)>0, \quad b_{\omega} \circ \tilde{\pi} \circ \varphi_{t_{2}}^{ \pm}(x, \eta) \neq 0,
\end{aligned}
$$

where $\tilde{\pi}: S^{*} \Omega \rightarrow \Omega$ is the natural projection. Moreover, we have $T_{\omega \rightarrow \mathcal{O} \rightarrow \omega}=\max \left(T_{\omega \rightarrow \mathcal{O} \rightarrow \omega}^{+}, T_{\omega \rightarrow \mathcal{O} \rightarrow \omega}^{-}\right)$.

In what follows, for the sake of concision, we shall omit the projection $\tilde{\pi}$ when composing functions on $\Omega$ with the flows $\varphi^{ \pm}$, i.e., we shall write $b \circ \varphi_{t_{1}}^{ \pm}(x, \eta)$ in place of $b \circ \tilde{\pi} \circ \varphi_{t_{1}}^{ \pm}(x, \eta)$.

\subsection{Reformulation of the system in symmetric spaces}

As one can see, work in asymmetric spaces can be awkward. We thus set $w_{1}=(1-\Delta)^{-\frac{1}{2}} v_{1}, w_{2}=v_{2}$. Having $\left(v_{1}, v_{2}\right)$ solution to (1.6) is then equivalent to having $\left(w_{1}, w_{2}\right)$ solution of

$$
\begin{cases}P w_{1}=0 & \text { in }(0, T) \times \Omega, \\ P w_{2}=-b(x)(1-\Delta)^{\frac{1}{2}} w_{1} & \text { in }(0, T) \times \Omega,\end{cases}
$$

as $P$ and $(1-\Delta)^{-\frac{1}{2}}$ commute. Hence, System (1.1) is exactly controllable in time $T$ if and only if the inequality

$$
E_{0}\left(w_{1}(0)\right)+E_{0}\left(w_{2}(0)\right) \leq C \int_{0}^{T} \int_{\omega}\left|b_{\omega} w_{2}\right|^{2} d x d t
$$

is satisfied for all $\left(w_{1}, w_{2}\right) \in \mathscr{C}^{0}\left([0, T] ; L^{2}\left(\Omega ; \mathbb{C}^{2}\right)\right) \cap \mathscr{C}^{0}\left([0, T] ; H^{-1}\left(\Omega ; \mathbb{C}^{2}\right)\right)$ solutions of System $(2.6)$. Note that the observability inequality $(2.7)$ corresponds also to the exact controllability of the following system

$$
\begin{cases}P z_{1}+(1-\Delta)^{\frac{1}{2}} b(x) z_{2}=0 & \text { in }(0, T) \times \Omega, \\ P z_{2}=b_{\omega}(x) f & \text { in }(0, T) \times \Omega,\end{cases}
$$


with initial and final data in $H^{1}\left(\Omega ; \mathbb{C}^{2}\right) \times L^{2}\left(\Omega ; \mathbb{C}^{2}\right)$ (see Section 5.1 for details). Connexion with System (1.1) is obtained by setting $z_{1}=(1-\Delta)^{\frac{1}{2}} u_{1}$ and $z_{2}=u_{2}$.

To prove the well-posedness of System (2.6), we introduce the space

$$
\mathscr{H}=L^{2}\left(\Omega ; \mathbb{C}^{2}\right) \times H^{-1}\left(\Omega ; \mathbb{C}^{2}\right),
$$

endowed with the natural inner product.

Proposition 2.5. For any $\left(w_{1}^{0}, w_{2}^{0}, w_{1}^{1}, w_{2}^{1}\right) \in \mathscr{H}$ and any $T>0$, System (2.6) with

$$
\left.\left(w_{1}, w_{2}, \partial_{t} w_{1}, \partial_{t} w_{2}\right)\right|_{t=0}=\left(w_{1}^{0}, w_{2}^{0}, w_{1}^{1}, w_{2}^{1}\right),
$$

has a unique solution $\left(w_{1}, w_{2}\right) \in \mathscr{C}^{0}\left(-T, T ; L^{2}\left(\Omega ; \mathbb{C}^{2}\right)\right) \cap \mathscr{C}^{1}\left(-T, T ; H^{-1}\left(\Omega ; \mathbb{C}^{2}\right)\right)$, depending continuously on $\left(w_{1}^{0}, w_{2}^{0}, w_{1}^{1}, w_{2}^{1}\right)$, i.e.

$$
\sup _{t \in(-T, T)}\left\{E_{0}\left(w_{1}(t)\right)+E_{0}\left(w_{2}(t)\right)\right\} \leq C(T)\left(\left\|w_{1}^{0}\right\|_{L^{2}(\Omega)}^{2}+\left\|w_{1}^{1}\right\|_{H^{-1}(\Omega)}^{2}+\left\|w_{2}^{0}\right\|_{L^{2}(\Omega)}^{2}+\left\|w_{2}^{1}\right\|_{H^{-1}(\Omega)}^{2}\right) .
$$

System (2.6) can be written as the first-order system

$$
\partial_{t} \mathscr{W}+\mathscr{A} \mathscr{W}=0,
$$

where $\mathscr{W}={ }^{t}\left(w_{1}, w_{2}, \partial_{t} w_{1}, \partial_{t} w_{2}\right)$ and the operator $\mathscr{A}$ is given by

$$
\mathscr{A}=\left(\begin{array}{cccc}
0 & 0 & -\mathrm{Id} & 0 \\
0 & 0 & 0 & -\mathrm{Id} \\
-\Delta & 0 & 0 & 0 \\
b(1-\Delta)^{\frac{1}{2}} & -\Delta & 0 & 0
\end{array}\right), \quad D(\mathscr{A})=H^{1}\left(\Omega ; \mathbb{C}^{2}\right) \times L^{2}\left(\Omega ; \mathbb{C}^{2}\right) .
$$

The Lumer-Phillips Theorem [Paz83] can be applied to (2.10) for positive and negative times $t$ since the operators $\lambda_{0} \operatorname{Id} \pm \mathscr{A}$ are maximal monotone for $\lambda_{0}$ sufficiently large (due to the cascade structure of the system). Hence $-\mathscr{A}$ generates a strongly continuous group that we shall denote by $\left(e^{-t \mathscr{A}}\right)_{t \in \mathbb{R}}$.

At places, we shall also write System (2.6) in the form

$$
\mathcal{P} W=0, \quad W=\left(w_{1}, w_{2}\right)^{T},
$$

with

$$
\mathcal{P}=\left(\begin{array}{cc}
P & 0 \\
B & P
\end{array}\right) \in \Psi_{\text {phg }}^{2}\left(M ; \mathbb{C}^{2 \times 2}\right), \quad \text { and } B=b(x)(1-\Delta)^{\frac{1}{2}} .
$$

According to [See67] or [Shu01, Theorem 11.2], we have $B \in \Psi_{\text {phg }}^{1}(\Omega ; \mathbb{C})$, with principal symbol $\sigma_{1}(B)(x, \eta)=b|\eta|_{x}$.

\section{Observability for $T>T_{\omega \rightarrow \mathcal{O} \rightarrow \omega}$}

In this section, we prove the following theorem.

Proposition 3.1. Suppose that $T>T_{\omega \rightarrow \mathcal{O} \rightarrow \omega}$. Then, the observability inequality (2.7) holds for any $\mathscr{C}^{0}\left(0, T ; L^{2}\left(\Omega ; \mathbb{C}^{2}\right)\right) \cap \mathscr{C}^{1}\left(0, T ; H^{-1}\left(\Omega ; \mathbb{C}^{2}\right)\right)$-solution of $(2.6)$.

The positive controllability result of Theorem 1.3 is then a direct consequence of Theorem 3.1.

To prove Proposition 3.1, we follow the compactness-uniqueness method of [RT74, BLR92, Bur97a], which consists in two steps. First we prove the observability inequality (2.7) in a weaker form, with an additional compact terms on the right hand-side. This allows one to handle high frequencies. Second, we use a uniqueness argument to handle low frequencies and conclude the proof of the observability inequality (2.7). 


\subsection{A relaxed observability inequality}

We shall prove the following result.

Proposition 3.2. Suppose that $T>T_{\omega \rightarrow \mathcal{O} \rightarrow \omega}$. Then, the observability inequality

$$
E_{0}\left(w_{1}(0)\right)+E_{0}\left(w_{2}(0)\right) \leq C\left(\int_{0}^{T} \int_{\omega}\left|b_{\omega} w_{2}\right|^{2} d x d t+E_{-1}\left(w_{1}(0)\right)+E_{-1}\left(w_{2}(0)\right)\right)
$$

holds for any $\mathscr{C}^{0}\left(0, T ; L^{2}\left(\Omega ; \mathbb{C}^{2}\right)\right) \cap \mathscr{C}^{1}\left(0, T ; H^{-1}\left(\Omega ; \mathbb{C}^{2}\right)\right)$-solution of $(2.6)$.

Proof. We proceed by contradiction and suppose that the observability inequality (3.1) is not satisfied. Thus, there exists a sequence $\left(w_{1}^{k}, w_{2}^{k}\right)_{k \in \mathbb{N}}$ of $\mathscr{C}^{0}\left(0, T ; L^{2}(\Omega)\right) \cap \mathscr{C}^{1}\left(0, T ; H^{-1}(\Omega)\right)$-solutions of

$$
\begin{cases}P w_{1}^{k}=0 & \text { in }(0, T) \times \Omega \\ P w_{2}^{k}+B w_{1}^{k}=0 & \text { in }(0, T) \times \Omega\end{cases}
$$

such that

$$
\begin{aligned}
& E_{0}\left(w_{1}^{k}(0)\right)+E_{0}\left(w_{2}^{k}(0)\right)=1, \\
& \int_{0}^{T} \int_{\omega}\left|b_{\omega} w_{2}^{k}\right|^{2} d x d t \rightarrow 0, \quad k \rightarrow \infty, \\
& E_{-1}\left(w_{1}^{k}(0)\right)+E_{-1}\left(w_{2}^{k}(0)\right) \rightarrow 0, \quad k \rightarrow \infty .
\end{aligned}
$$

According to (3.3) and the continuity of the solution with respect to the initial data, the sequence $\left(w_{1}^{k}, w_{2}^{k}\right)$ is bounded in $L^{2}\left(M_{T} ; \mathbb{C}^{2}\right)$. According to $(3.5)$, we have $\left(w_{1}^{k}(0), w_{2}^{k}(0), \partial_{t} w_{1}^{k}(0), \partial_{t} w_{2}^{k}(0)\right) \rightarrow$ $(0,0,0,0)$ in $L^{2}\left(\Omega ; \mathbb{C}^{2}\right) \times H^{-1}\left(\Omega ; \mathbb{C}^{2}\right)$. The continuity of the solution with respect to the initial data yields

$$
\left(w_{1}^{k}, w_{2}^{k}\right) \rightarrow(0,0) \quad \text { in } L^{2}\left(M_{T} ; \mathbb{C}^{2}\right) .
$$

As a consequence of [Gér91, Theorem 1], there exists a subsequence of $\left(W^{k}\right)_{k \in \mathbb{N}}=\left(w_{1}^{k}, w_{2}^{k}\right)_{k \in \mathbb{N}}($ still denoted $\left(W^{k}\right)_{k \in \mathbb{N}}=\left(w_{1}^{k}, w_{2}^{k}\right)_{k \in \mathbb{N}}$ in what follows) and a microlocal defect measure

$$
\mu=\left(\begin{array}{ll}
\mu_{1} & \mu_{12} \\
\bar{\mu}_{12} & \mu_{2}
\end{array}\right) \in \mathcal{M}_{+}\left(S^{*} M_{T} ; \mathbb{C}^{2 \times 2}\right),
$$

(according to [Tar90, Gér91], see also [Bur97b, Proposition 5], this measure is intrinsically defined on $\left.S^{*} M_{T}\right)$ such that for any $\mathcal{A} \in \Psi_{\mathrm{phg}}^{0}\left(M_{T} ; \mathbb{C}^{2 \times 2}\right)$ (recall that symbols are compactly supported in time $t$ here, see Section 2.1),

$$
\lim _{k \rightarrow \infty}\left(\mathcal{A} W^{k}, W^{k}\right)_{L^{2}\left(M_{T} ; \mathbb{C}^{2}\right)}=\int_{S^{*} M_{T}} \operatorname{tr}\left\{\sigma_{0}(\mathcal{A})(\rho) \mu(d \rho)\right\}
$$

Testing the measure $\mu$ on different operators $\mathcal{A}$, the limit equation (3.6) can be equivalently written as

$$
\left\{\begin{array}{l}
\lim _{k \rightarrow \infty}\left(A w_{1}^{k}, w_{1}^{k}\right)_{L^{2}\left(M_{T} ; \mathbb{C}\right)}=\left\langle\mu_{1}, \sigma_{0}(A)\right\rangle_{S^{*} M_{T}}, \\
\lim _{k \rightarrow \infty}\left(A w_{2}^{k}, w_{2}^{k}\right)_{L^{2}\left(M_{T} ; \mathbb{C}\right)}=\left\langle\mu_{2}, \sigma_{0}(A)\right\rangle_{S^{*} M_{T}}, \\
\lim _{k \rightarrow \infty}\left(A w_{1}^{k}, w_{2}^{k}\right)_{L^{2}\left(M_{T} ; \mathbb{C}\right)}=\left\langle\mu_{12}, \sigma_{0}(A)\right\rangle_{S^{*} M_{T}},
\end{array}\right.
$$

for any $A \in \Psi_{\text {phg }}^{0}\left(M_{T} ; \mathbb{C}\right)$.

The following lemma gives the properties of the three measures $\mu_{1}, \mu_{2}$ and $\mu_{12}$, and is a key point in the proof of Proposition 3.2. 
Lemma 3.3 (Properties of the measure $\mu$ ). 1. Suppose that the sequence $\left(W^{k}\right)_{k \in \mathbb{N}}$ satisfies $(3.2)$ and (3.5). Then, we have $\mu_{1}, \mu_{2} \in \mathcal{M}_{+}\left(S^{*} M_{T}\right), \operatorname{supp}\left(\mu_{1}\right) \subset \operatorname{Char}(P), \operatorname{supp}\left(\mu_{2}\right) \subset \operatorname{Char}(P)$. Moreover, $\operatorname{supp}\left(\mu_{12}\right) \subset \operatorname{supp}\left(\mu_{1}\right) \cap \operatorname{supp}\left(\mu_{2}\right) \subset \operatorname{Char}(P)$. Finally these three measures satisfy the equations

$$
\left\{\begin{array}{l}
\left\langle\mu_{1}, H_{p} a\right\rangle_{S^{*} M_{T}}=0, \\
\left\langle\mu_{2}, H_{p} a\right\rangle_{S^{*} M_{T}}=-\left\langle\operatorname{Im}\left(\mu_{12}\right), 2 b|\eta|_{x} a\right\rangle_{S^{*} M_{T}}, \\
\left\langle\operatorname{Im}\left(\mu_{12}\right), H_{p} a\right\rangle_{S^{*} M_{T}}=-\left\langle\mu_{1}, b|\eta|_{x} a\right\rangle_{S^{*} M_{T}}, \\
\left\langle\operatorname{Re}\left(\mu_{12}\right), H_{p} a\right\rangle_{S^{*} M_{T}}=0
\end{array}\right.
$$

for any $a \in S_{\mathrm{phg}}^{-1}\left(T^{*} M_{T} ; \mathbb{C}\right)$.

2. If moreover the sequence $\left(W^{k}\right)_{k \in \mathbb{N}}$ satisfies (3.4), then we also have $\operatorname{supp}\left(\mu_{2}\right) \cap \pi^{-1}((0, T) \times \omega)=$ $\emptyset$ and $\operatorname{supp}\left(\mu_{12}\right) \cap \pi^{-1}((0, T) \times \omega)=\emptyset$.

The proof of this lemma is given in Appendix B.1.

We shall prove below that the first and the last equations in (3.8) yield a "free" propagation result for the measures $\mu_{1}$ and $\operatorname{Re}\left(\mu_{12}\right)$. In particular, since $\mu_{12}=0$ on $\pi^{-1}((0, T) \times \omega)$, and $T>T_{\omega \rightarrow \mathcal{O} \rightarrow \omega} \geq T_{\omega}$, this will give $\operatorname{Re}\left(\mu_{12}\right)=0$.

The most important equation in (3.8) is the third one, that is to be viewed as a transport equation for the measure $\operatorname{Im}\left(\mu_{12}\right)$.

We already know that the supports of the three measures are subsets of $\operatorname{Char}(P) \cap S^{*} M_{T}$. Let us pick $\rho_{*}=\left(t_{*}, x_{*}, \tau_{*}, \eta_{*}\right) \in \operatorname{Char}(P) \cap S^{*} M_{T}$. According to Definition 2.3, there exist $0<t_{0}<$ $t_{1}<t_{2}<T$ such that

$$
\phi_{t_{0}-t_{*}}\left(\rho_{*}\right) \in \pi^{-1}((0, T) \times \omega), \quad \phi_{t_{1}-t_{*}}\left(\rho_{*}\right) \in \pi^{-1}((0, T) \times \mathcal{O}), \quad \phi_{t_{2}-t_{*}}\left(\rho_{*}\right) \in \pi^{-1}((0, T) \times \omega) .
$$

We take three open subsets $\theta_{0}, \theta_{1}, \theta_{2} \subset S^{*} M_{T}$, satisfying

$$
\begin{gathered}
\rho_{j}=\phi_{t_{j}-t_{*}}\left(\rho_{*}\right) \in \theta_{j} \text { for } j=0,1,2, \\
\pi\left(\theta_{0}\right) \subset(0, T) \times \omega, \quad \pi\left(\theta_{1}\right) \subset(0, T) \times \mathcal{O}, \quad \pi\left(\theta_{2}\right) \subset(0, T) \times \omega .
\end{gathered}
$$

This geometrical situation is illustrated in Figure 1.

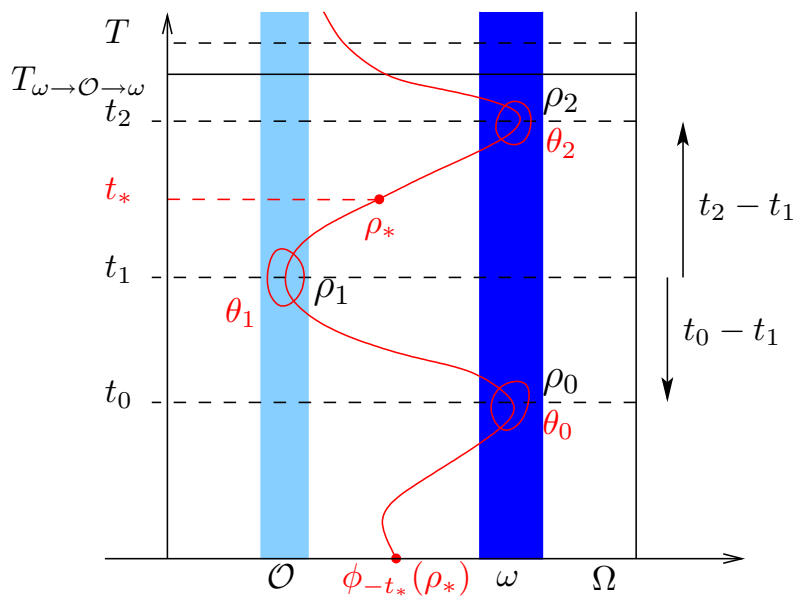

Figure 1: Geometrical situation in the case $T>T_{\omega \rightarrow \mathcal{O} \rightarrow \omega}$.

We now choose a function $e \in \mathscr{C}_{c}^{\infty}\left(S^{*} M_{T}\right)$ such that

$$
\operatorname{supp}(e) \subset \phi_{t_{1}-t_{0}}\left(\theta_{0}\right) \cap \theta_{1} \cap \phi_{t_{1}-t_{2}}\left(\theta_{2}\right), \quad e \geq 0, \quad \text { and } \quad e\left(\rho_{1}\right)=1 .
$$


Note that the set $\phi_{t_{1}-t_{0}}\left(\theta_{0}\right) \cap \theta_{1} \cap \phi_{t_{1}-t_{2}}\left(\theta_{2}\right)$ is open since $\phi_{t}: S^{*} M_{T} \rightarrow S^{*} M_{T}$ is bicontinuous and is nonempty since it contains $\rho_{1}$, according to (3.9).

Now, we apply the third identity of (3.8) to $a=e \circ \phi_{s} \in \mathscr{C}_{c}^{\infty}\left(S^{*} M_{T}\right)$ (which can be extended as a symbol, namely $\tilde{\lambda}^{-1} a(t, x, \tau / \tilde{\lambda}, \eta / \tilde{\lambda}) \in S_{\text {phg }}^{-1}\left(T^{*} M_{T} ; \mathbb{R}\right)$, where $\tilde{\lambda}$ is defined in $\left.(2.2)\right)$ for $s \in$ $\left(t_{1}-t_{2}, t_{1}-t_{0}\right)$, as a consequence of (3.9)-(3.10). ¿From the third equation in (3.8) we obtain

$$
\begin{aligned}
-\left\langle\mu_{1}, b|\eta|_{x} e \circ \phi_{s}\right\rangle_{S^{*} M_{T}} & =\left\langle\operatorname{Im}\left(\mu_{12}\right), H_{p}\left(e \circ \phi_{s}\right)\right\rangle_{S^{*} M_{T}}, \\
& =\left\langle\operatorname{Im}\left(\mu_{12}\right), \frac{d}{d s} e \circ \phi_{s}\right\rangle_{S^{*} M_{T}}, \\
& =\frac{d}{d s}\left\langle\operatorname{Im}\left(\mu_{12}\right), e \circ \phi_{s}\right\rangle_{S^{*} M_{T}} .
\end{aligned}
$$

Integrating this equation on the interval $\left(t_{1}-t_{2}, t_{1}-t_{0}\right)$ gives

$$
-\int_{t_{1}-t_{2}}^{t_{1}-t_{0}}\left\langle\mu_{1}, b|\eta|_{x} e \circ \phi_{s}\right\rangle_{S^{*} M_{T}} d s=\left\langle\operatorname{Im}\left(\mu_{12}\right), e \circ \phi_{t_{1}-t_{0}}\right\rangle_{S^{*} M_{T}}-\left\langle\operatorname{Im}\left(\mu_{12}\right), e \circ \phi_{t_{1}-t_{2}}\right\rangle_{S^{*} M_{T}} .
$$

Moreover, from (3.10), we have $\operatorname{supp}\left(e \circ \phi_{t_{1}-t_{2}}\right) \subset \theta_{2}$ and $\operatorname{supp}\left(e \circ \phi_{t_{1}-t_{0}}\right) \subset \theta_{0}$. Since $\pi\left(\theta_{0}\right), \pi\left(\theta_{2}\right) \subset$ $(0, T) \times \omega$ and $\pi\left(\operatorname{supp}\left(\mu_{12}\right)\right) \cap(0, T) \times \omega=\emptyset$ from Lemma 3.3, the right hand-side of (3.11) vanishes, and we obtain

$$
\int_{t_{1}-t_{2}}^{t_{1}-t_{0}}\left\langle\mu_{1}, b|\eta|_{x} e \circ \phi_{s}\right\rangle_{S^{*} M_{T}} d s=0 .
$$

In this expression, the measure $\mu_{1}$ and the functions $|\eta|_{x}, b$ and $e \circ \phi_{s}$ are non-negative on the interval $\left(t_{1}-t_{2}, t_{1}-t_{0}\right)$. We thus obtain, for any $s \in\left(t_{1}-t_{2}, t_{1}-t_{0}\right)$,

$$
\left\langle\mu_{1}, b|\eta|_{x} e \circ \phi_{s}\right\rangle_{S^{*} M_{T}}=0,
$$

and in particular for $s=0 \in\left(t_{1}-t_{2}, t_{1}-t_{0}\right)$ we find

$$
\left\langle\mu_{1}, b|\eta|_{x} e\right\rangle_{S^{*} M_{T}}=0 .
$$

We have $b\left(\pi\left(\rho_{1}\right)\right)>0$ since $\pi\left(\rho_{1}\right) \in(0, T) \times \mathcal{O}$, and $e\left(\rho_{1}\right)=1$. We hence have

$$
\mu_{1} \text { vanishes in a neighborhood of } \rho_{1} \text {. }
$$

We can now prove that $\mu_{1}$ is identically zero on this bicharacteristic. This is a direct consequence of the first equation of (3.8): applied to $a \circ \phi_{s}$ in place of $a$ as long as $\operatorname{supp}\left(a \circ \phi_{s}\right) \subset S^{*} M_{T}$, it yields

$$
0=\left\langle\mu_{1}, H_{p} a \circ \phi_{s}\right\rangle_{S^{*} M_{T}}=\left\langle\mu_{1}, \frac{d}{d s} a \circ \phi_{s}\right\rangle_{S^{*} M_{T}}=\frac{d}{d s}\left\langle\mu_{1}, a \circ \phi_{s}\right\rangle_{S^{*} M_{T}} .
$$

This direclty gives

$$
\begin{gathered}
\left\langle\mu_{1}, a \circ \phi_{s}\right\rangle_{S^{*} M_{T}}=\left\langle\mu_{1}, a\right\rangle_{S^{*} M_{T}}, \quad \text { for all } a \in S_{\text {phg }}^{-1}\left(T^{*} M_{T} ; \mathbb{C}\right), \\
\text { for all } s \in \mathbb{R} \text { such that } \operatorname{supp}\left(a \circ \phi_{s}\right) \subset S^{*} M_{T} .
\end{gathered}
$$

¿From the argument above, there exists $a \in S_{\mathrm{phg}}^{-1}\left(T^{*} M_{T} ; \mathbb{C}\right)$ with a small support in a neighborhood of $\rho_{1}$ and $a\left(\rho_{1}\right)=1$, such that $\left\langle\mu_{1}, a\right\rangle_{S^{*} M_{T}}=0$. Taking $s=t_{1}-t_{*}$ in (3.13) then yields

$$
\left\langle\mu_{1}, a \circ \phi_{t_{1}-t_{*}}\right\rangle_{S^{*} M_{T}}
$$

with $a \circ \phi_{t_{1}-t_{*}}\left(\rho_{*}\right)=1$. This implies that $\mu_{1}$ vanishes in a neighborhood of $\rho_{*}$. Here $\rho_{*}$ was chosen arbitrary in $S^{*} M_{T}$. We thus have $\mu_{1}=0$ on $S^{*} M_{T}$.

The third equation of $(3.8)$ then becomes $\left\langle\operatorname{Im}\left(\mu_{12}\right), H_{p} a\right\rangle_{S^{*} M_{T}}=0$ for all $a \in S_{\text {phg }}^{-1}\left(T^{*} M_{T} ; \mathbb{C}\right)$. The same analysis direcly yields the propagation of the measure $\operatorname{Im}\left(\mu_{12}\right)$. This, together with $\operatorname{supp}\left(\operatorname{Im}\left(\mu_{12}\right)\right) \cap \pi^{-1}((0, T) \times \omega)=\emptyset$ gives $\operatorname{Im}\left(\mu_{12}\right)=0$ on $S^{*} M_{T}$, as $T>T_{\omega \rightarrow \mathcal{O} \rightarrow \omega} \geq T_{\omega}$. Similarly, we obtain $\operatorname{Re}\left(\mu_{12}\right)=0$ on $S^{*} M_{T}$ with the last equation of (3.8). Finally, the second equation of (3.8) 
now reads $\left\langle\mu_{2}, H_{p} a\right\rangle_{S^{*} M_{T}}=0$ for all $a \in S_{\mathrm{phg}}^{-1}\left(T^{*} M_{T} ; \mathbb{C}\right)$. This implies $\mu_{2}=0$ on $S^{*} M_{T}$ as we already know that $\operatorname{supp}\left(\mu_{2}\right) \cap \pi^{-1}((0, T) \times \omega)=\emptyset$ from Lemma 3.3 .

Since $\mu=0$, we have

$$
\left(w_{1}^{k}, w_{2}^{k}\right) \rightarrow(0,0) \quad \text { strongly in } L_{\mathrm{loc}}^{2}\left(M_{T} ; \mathbb{C}^{2}\right) .
$$

Let us take $\chi \in \mathscr{C}_{c}^{\infty}(0, T ; \mathbb{C})$, multiply the second equation in $(3.2)$ by $\chi(1-\Delta)^{-1} w_{2}^{k}$ and integrate on $(0, T) \times \Omega$. After an integration by parts in time, this gives

$$
\begin{array}{rl}
\int_{0}^{T} \int_{\Omega} \chi\left|(1-\Delta)^{-\frac{1}{2}} \partial_{t} w_{2}^{k}\right|^{2} & d x d t+\int_{0}^{T} \int_{\Omega} \partial_{t} \chi \partial_{t} w_{2}^{k}(1-\Delta)^{-1} w_{2}^{k} d x d t \\
+\int_{0}^{T} \int_{\Omega} \chi \Delta w_{2}^{k}(1-\Delta)^{-1} w_{2}^{k} d x d t=\int_{0}^{T} \int_{\Omega} \chi B w_{1}^{k}(1-\Delta)^{-1} w_{2}^{k} d x d t .
\end{array}
$$

In this expression, we have

$$
\begin{gathered}
\int_{0}^{T} \int_{\Omega} \partial_{t} \chi \partial_{t} w_{2}^{k}(1-\Delta)^{-1} w_{2}^{k} \rightarrow 0 \quad \text { since } \partial_{t} \chi \partial_{t}(1-\Delta)^{-1} \in \Psi_{\text {phg }}^{-1}\left(M_{T} ; \mathbb{C}\right), \\
\int_{0}^{T} \int_{\Omega} \chi B w_{1}^{k}(1-\Delta)^{-1} w_{2}^{k} \rightarrow 0 \quad \text { since } \chi(1-\Delta)^{-1} B \in \Psi_{\text {phg }}^{-1}\left(M_{T} ; \mathbb{C}\right),
\end{gathered}
$$

and

$$
\int_{0}^{T} \int_{\Omega} \chi \Delta w_{2}^{k}(1-\Delta)^{-1} w_{2}^{k} \rightarrow\left\langle\mu_{2}, \chi\right\rangle_{S^{*} M_{T}}=0
$$

As a consequence, we obtain, for all $\chi \in \mathscr{C}_{c}^{\infty}(0, T ; \mathbb{C})$

$$
\int_{0}^{T} \int_{\Omega} \chi\left|(1-\Delta)^{-\frac{1}{2}} \partial_{t} w_{2}^{k}\right|^{2} \rightarrow 0
$$

This, together with (3.14) yields, for all $0<\varepsilon_{1}<\varepsilon_{2}<T$,

$$
\int_{\varepsilon_{1}}^{\varepsilon_{2}} E_{0}\left(w_{2}^{k}(t)\right) d t \rightarrow 0
$$

The same method with the first equation of (3.2) also gives $\int_{\varepsilon_{1}}^{\varepsilon_{2}} E_{0}\left(w_{1}^{k}(t)\right) d t \rightarrow 0$. Hence, $E_{0}\left(w_{2}^{k}(t)\right)+$ $E_{0}\left(w_{1}^{k}(t)\right) \rightarrow 0$ for almost every $t \in\left(\varepsilon_{1}, \varepsilon_{2}\right)$. Picking such a time, say $t_{3}$, the (backward) wellposedness result of Proposition 2.5 for the Cauchy problem (3.2) with data given at $t_{3}$ gives

$$
E_{0}\left(w_{1}^{k}(0)\right)+E_{0}\left(w_{2}^{k}(0)\right) \leq C\left(E_{0}\left(w_{1}^{k}\left(t_{3}\right)\right)+E_{0}\left(w_{2}^{k}\left(t_{3}\right)\right)\right) .
$$

This yields $E_{0}\left(w_{1}^{k}(0)\right)+E_{0}\left(w_{2}^{k}(0)\right) \rightarrow 0$, gives a contradiction with (3.3), and concludes the proof of the proposition.

\subsection{End of the proof of Proposition 3.1}

With the relaxed observability inequality of Proposition 3.2, we are now able to handle the lowfrequencies and conlude the proof Theorem 3.1. The main point here is a unique continuation result for solutions of the elliptic problem associated with System (2.6). The idea of reducing the observability for the low frequencies to an elliptic unique continuation result, and associated technology are due to [BLR92]. Here we follow the expository lectures [BG02].

We first define for any $T>0$ the set of invisible solutions (see [BLR92]) from $(0, T) \times \omega$ :

$$
\begin{aligned}
\mathcal{N}(T)=\{\mathscr{W}= & \left(w_{1}^{0}, w_{2}^{0}, w_{1}^{1}, w_{2}^{1}\right) \in \mathscr{H} \text { such that the associated solution of }(2.6) \\
& \text { satisfies } \left.w_{2}(t, x)=0 \text { for all }(t, x) \in(0, T) \times \omega\right\},
\end{aligned}
$$

We have the following key lemma, which is proved at the end of this section. 
Lemma 3.4. For $T>T_{\omega \rightarrow \mathcal{O} \rightarrow \omega}$, we have $\mathcal{N}(T)=\{0\}$.

As for the proof of the relaxed observability inequality of Proposition 3.2 we proceed by contradiction. We suppose that the result of Theorem 3.1 is false. Thus, there exists a sequence $\left(w_{1}^{k}, w_{2}^{k}\right)_{k \in \mathbb{N}}$ of $\mathscr{C}^{0}\left(0, T ; L^{2}(\Omega)\right) \cap \mathscr{C}^{1}\left(0, T ; H^{-1}(\Omega)\right)$-solutions of (2.6) such that

$$
\begin{gathered}
E_{0}\left(w_{1}^{k}(0)\right)+E_{0}\left(w_{2}^{k}(0)\right)=1, \\
\int_{0}^{T} \int_{\omega}\left|b_{\omega} w_{2}^{k}\right|^{2} d x d t \rightarrow 0 .
\end{gathered}
$$

Equation (3.15) and Proposition 2.5 imply that the sequence $\left(w_{1}^{k}, w_{2}^{k}\right)_{k \in \mathbb{N}}$ is bounded in $L^{2}\left(M_{T} ; \mathbb{C}^{2}\right)$. Hence, there exists a subsequence (also denoted $\left(w_{1}^{k}, w_{2}^{k}\right)_{k \in \mathbb{N}}$ in what follows) weakly converging in $L^{2}\left(M_{T} ; \mathbb{C}^{2}\right)$, towards $\left(w_{1}, w_{2}\right) \in L^{2}\left(M_{T} ; \mathbb{C}^{2}\right)$. From $(3.16)$, this limit satisfies, for all $t \in(0, T)$, $\left.w_{2}\right|_{\omega}=0$ and is moreover a solution of (2.6). Hence, we have $\left(w_{1}(0), w_{2}(0), \partial_{t} w_{1}(0), \partial_{t} w_{2}(0)\right) \in \mathcal{N}(T)$. According to Lemma 3.4, this yields $\left(w_{1}, w_{2}\right)=(0,0)$. Besides, the imbedding $\mathscr{H} \hookrightarrow H^{-1}(\Omega)^{2} \times$ $H^{-2}(\Omega)^{2}$ is compact. This yields

$$
E_{-1}\left(w_{1}^{k}(0)\right)+E_{-1}\left(w_{2}^{k}(0)\right) \rightarrow E_{-1}\left(w_{1}(0)\right)+E_{-1}\left(w_{2}(0)\right) .
$$

The relaxed observability inequality (3.1) hence yields

$$
1 \leq C\left(E_{-1}\left(w_{1}(0)\right)+E_{-1}\left(w_{2}(0)\right)\right),
$$

which contradicts the fact that $\left(w_{1}, w_{2}\right)=(0,0)$, and concludes the proof of the Proposition 3.1.

It only remains to prove Lemma 3.4.

Proof of Lemma 3.4. First, Proposition 2.5 implies that $\mathcal{N}(T)$ is a closed subspace of $\mathscr{H}$. Second, applying the relaxed observability inequality (3.1) to an element of $\mathcal{N}(T)$ gives

$$
\|\mathscr{W}\|_{\mathscr{H}}=E_{0}\left(w_{1}(0)\right)+E_{0}\left(w_{2}(0)\right) \leq C\left(E_{-1}\left(w_{1}(0)\right)+E_{-1}\left(w_{2}(0)\right)\right) .
$$

Using the compact imbedding $\mathscr{H} \hookrightarrow H^{-1}(\Omega)^{2} \times H^{-2}(\Omega)^{2}$, this implies that $\mathcal{N}(T)$ has a finite dimension, and is thus complete for any norm. Moreover, setting $\delta=\frac{1}{2}\left(T-T_{\omega \rightarrow \mathcal{O} \rightarrow \omega}\right)>0$, we remark that (3.17) is also satisfied by all $\mathscr{W} \in \mathcal{N}(T-\delta)$. Taking $\mathscr{W} \in \mathcal{N}(T)$ implies that, for all $\varepsilon \in(0, \delta)$, we have $e^{-\varepsilon \mathscr{A} \mathscr{W}} \in \mathcal{N}(T-\delta)$. We also have, for $\lambda_{0}$ sufficiently large, according to Section 2.3,

$$
\left(\lambda_{0}+\mathscr{A}\right)^{-1} \frac{1}{\varepsilon}\left(\operatorname{Id}-e^{-\varepsilon \mathscr{A}}\right) \mathscr{W}=\frac{1}{\varepsilon}\left(\operatorname{Id}-e^{-\varepsilon \mathscr{A}}\right)\left(\lambda_{0}+\mathscr{A}\right)^{-1} \mathscr{W} \underset{\varepsilon \rightarrow 0^{+}}{\rightarrow} \mathscr{A}\left(\lambda_{0}+\mathscr{A}\right)^{-1} \mathscr{W} \quad \text { in } \mathscr{H},
$$

as $\left(\lambda_{0}+\mathscr{A}\right)^{-1} \mathscr{W} \in D(\mathscr{A})$. As a consequence, the sequence $\left(\frac{1}{\varepsilon}\left(\mathrm{Id}-e^{-\varepsilon \mathscr{A}}\right) \mathscr{W}\right)_{\varepsilon>0}$ is a Cauchy sequence in $\mathcal{N}(T-\delta)$, endowed with the norm $\left\|\left(\lambda_{0}+\mathscr{A}\right)^{-1} \cdot\right\| \mathscr{H}$. As all norms are equivalent, the sequence $\left(\frac{1}{\varepsilon}\left(\operatorname{Id}-e^{-\mathscr{E} \mathscr{A}}\right) \mathscr{W}\right)_{\varepsilon>0}$ is thus also a Cauchy sequence in $\mathcal{N}(T-\delta)$, endowed with the norm $\|\cdot\|_{\mathscr{H}}$, which yields $\mathscr{A} \mathscr{W} \in \mathscr{H}$. Hence, we have $\mathcal{N}(T) \subset D(\mathscr{A})$. Denoting by $\tilde{\mathscr{W}}(t)$ the solution of

$$
\partial_{t} \tilde{\mathscr{W}}+\mathscr{A} \tilde{\mathscr{W}}=0,\left.\quad \tilde{\mathscr{W}}\right|_{t=0}=\mathscr{W}, \quad \text { with } \tilde{\mathscr{W}} \in \mathscr{C}^{1}([0, T] ; \mathscr{H}) \cap \mathscr{C}^{0}([0, T] ; D(\mathscr{A})),
$$

from semigroup theory, we remark that we have

$$
\mathscr{A} \mathscr{W}=\left.\partial_{t} \tilde{\mathscr{W}}\right|_{t=0} \in \mathcal{N}(T) \quad \text { if } \quad \mathscr{W} \in \mathcal{N}(T) .
$$

In fact, as $w_{2}(t, x)=0$ for all $(t, x) \in(0, T) \times \omega$, the same holds for $\partial_{t} w_{2}$. Consequently $\mathscr{A} \mathcal{N}(T) \subset$ $\mathcal{N}(T)$.

Since $\mathcal{N}(T)$ is a finite dimensional subspace of $D(\mathscr{A})$, stable by the action of the operator $\mathscr{A}$, it contains an eigenfunction of $\mathscr{A}$. There exist $\mu \in \mathbb{C}$ and $\mathscr{W}_{\mu} \in \mathcal{N}(T)$ such that $\mathscr{A} \mathscr{W}_{\mu}=\mu \mathscr{W}_{\mu}$. Writing $\mathscr{W}_{\mu}={ }^{t}\left(w_{1}^{0}, w_{2}^{0}, w_{1}^{1}, w_{2}^{1}\right)$, this is equivalent to having

$$
\left\{\begin{array}{l}
-w_{1}^{1}=\mu w_{1}^{0} \\
-w_{2}^{1}=\mu w_{2}^{0} \\
-\Delta w_{1}^{0}=\mu w_{1}^{1} \\
-\Delta w_{2}^{0}+b(x)(1-\Delta)^{\frac{1}{2}} w_{1}^{0}=\mu w_{2}^{1} .
\end{array}\right.
$$


This system implies

$$
\left\{\begin{array}{l}
-\Delta w_{1}^{0}=-\mu^{2} w_{1}^{0}, \\
-\Delta w_{2}^{0}+b(x)(1-\Delta)^{\frac{1}{2}} w_{1}^{0}=-\mu^{2} w_{2}^{0} .
\end{array}\right.
$$

The first equation gives $\mu=i \sqrt{\kappa}$ with $\kappa \in \operatorname{Sp}(-\Delta) \subset \mathbb{R}_{+}$and $w_{1}^{0}$ is an eigenfunction of $-\Delta$ associated to $\kappa$. Hence, taking the $L^{2}(\Omega)$-inner product of the first line of $(3.18)$ with $(1-\Delta)^{\frac{1}{2}} w_{2}^{0}$, and that of the second line of $(3.18)$ with $(1-\Delta)^{\frac{1}{2}} w_{1}^{0}$, we obtain

$$
\int_{\Omega} b(x)\left|(1-\Delta)^{\frac{1}{2}} w_{1}^{0}\right|^{2} d x=0 .
$$

Since $b \geq 0$ and $b$ does not vanish identically, this proves that $(1-\Delta)^{\frac{1}{2}} w_{1}^{0}=0$ on $\mathcal{O}$. As $(1-\Delta)^{\frac{1}{2}} w_{1}^{0}$ is an eigenfunction of the Laplace operator vanishing on $\mathcal{O}$, a unique continuation result (see for instance the classical reference [Aro57, AKS62], the book [Zui83] or the exposition article [LL11]) yields $(1-\Delta)^{\frac{1}{2}} w_{1}^{0}=0$ on $\Omega$. Hence $w_{1}^{0}=0$ on $\Omega$.

Moreover, $\mathscr{W} \in \mathcal{N}(T)$ yields $w_{2}^{0}=0$ on $\omega$. This proves that $w_{2}^{0}=0$ on $\Omega$, as $w_{2}^{0}$ is an eigenfunction of the Laplace operator as a consequence of (3.18). This concludes the proof of the Lemma 3.4.

Remark 3.5. Note that elliptic unique continuation properties as those used here are not known in general for $2 \times 2$ elliptic systems. For these types of general systems, such a result holds if $\omega \cap \mathcal{O} \neq \emptyset$ [Léa10, Proposition 5.1]. However the case $\omega \cap \mathcal{O}=\emptyset$ remains open in general.

Here, the cascade structure of System (2.6) allows us to bypass a more involved unique continuation theorem. We use that the eigenvalues and eigenfunctions of the operator

$$
\left(\begin{array}{cc}
-\Delta & 0 \\
b(1-\Delta)^{\frac{1}{2}} & -\Delta
\end{array}\right)
$$

are $\left(\kappa_{j},{ }^{t}\left(0, \varphi_{j}\right)\right)_{j \in \mathbb{N}}$, where $\left(\kappa_{j}, \varphi_{j}\right)_{j \in \mathbb{N}}$ are the eigenvalues and eigenfunctions of $-\Delta$. This is a very particular feature of cascade systems with twice the same elliptic operator on the diagonal.

\section{Lack of observability for $T<T_{\omega \rightarrow \mathcal{O} \rightarrow \omega}$}

In this section, we prove the following theorem.

Theorem 4.1. Suppose that $T<T_{\omega \rightarrow \mathcal{O} \rightarrow \omega}$. Then, there exists a bounded sequence $\left(w_{1}^{k}, w_{2}^{k}\right)_{k \in \mathbb{N}}$ of $\mathscr{C}^{0}\left(0, T ; L^{2}(\Omega)^{2}\right) \cap \mathscr{C}^{1}\left(0, T ; H^{-1}(\Omega)^{2}\right)$-solutions of (2.6) satisfying

$$
\left\{\begin{array}{l}
\liminf _{k \rightarrow \infty}\left(E_{0}\left(w_{1}^{k}(0)\right)+E_{0}\left(w_{2}^{k}(0)\right)\right) \geq 1, \\
\left(w_{1}^{k}, w_{2}^{k}\right) \rightarrow 0 \text { in } L^{2}\left(M_{T} ; \mathbb{C}^{2}\right)
\end{array}\right.
$$

such that the sequence $\left(w_{2}^{k}\right)_{k \in \mathbb{N}}$ is pure and its microlocal defect measure $\mu_{2}$ satisfies

$$
\left\langle\mu_{2}, \chi^{2}\left|b_{\omega}\right|^{2}\right\rangle_{S^{*} M_{T}}=0,
$$

for any $\chi \in \mathscr{C}_{c}^{\infty}(0, T)$.

We refer to [Gér91, Definition 1.3] for the definition of a pure sequence. As a direct consequence of this theorem, we have the following non-observability result.

Corollary 4.2. If $T<T_{\omega \rightarrow \mathcal{O} \rightarrow \omega}$, the observability inequality (2.7) does not hold.

The negative controllability result of Theorem 1.3 is then a direct consequence of Corollary 4.2 and HUM. 
Proof of Corollary 4.2. Suppose that the observability inequality (2.7) holds for some constant $C>$ 0 , for all solutions of (2.6). Then, for the sequence $\left(w_{1}^{k}, w_{2}^{k}\right)_{k \in \mathbb{N}}$ given by Theorem 4.1 , we have, for $k$ sufficiently large,

$$
\frac{1}{2} \leq E_{0}\left(w_{1}^{k}(0)\right)+E_{0}\left(w_{2}^{k}(0)\right) \leq K \int_{0}^{T} \int_{\omega}\left|b_{\omega} w_{2}^{k}\right|^{2} d x d t, \quad K>0 .
$$

The sequence $\left(w_{1}^{k}, w_{2}^{k}\right)_{k \in \mathbb{N}}$ being bounded in the energy space, we have in particular

$$
\int_{\omega}\left|b_{\omega} w_{2}^{k}(t, x)\right|^{2} d x \leq C_{0}, \quad \text { for some } C_{0}>0, \text { and all } k \in \mathbb{N}, t \in[0, T] \text {. }
$$

This yields, for any $\varepsilon \in\left(0, \frac{T}{2}\right)$,

$$
\begin{aligned}
\int_{0}^{T} \int_{\omega}\left|b_{\omega} w_{2}^{k}\right|^{2} d x d t & =\int_{0}^{\varepsilon} \int_{\omega}\left|b_{\omega} w_{2}^{k}\right|^{2} d x d t+\int_{\varepsilon}^{T-\varepsilon} \int_{\omega}\left|b_{\omega} w_{2}^{k}\right|^{2} d x d t+\int_{T-\varepsilon}^{T} \int_{\omega}\left|b_{\omega} w_{2}^{k}\right|^{2} d x d t \\
& \leq 2 \varepsilon C_{0}+\int_{\varepsilon}^{T-\varepsilon} \int_{\omega}\left|b_{\omega} w_{2}^{k}\right|^{2} d x d t \leq 2 \varepsilon C_{0}+\int_{0}^{T} \int_{\omega}\left|\chi b_{\omega} w_{2}^{k}\right|^{2} d x d t,
\end{aligned}
$$

with $\chi \in \mathscr{C}_{c}^{\infty}(0, T)$ such that $\chi=1$ on $(\varepsilon, T-\varepsilon)$. We fix $\varepsilon$ such that $2 \varepsilon C_{0}=\frac{1}{8 K}$ and obtain

$$
\int_{0}^{T} \int_{\omega}\left|b_{\omega} w_{2}^{k}\right|^{2} d x d t \leq \frac{1}{8 K}+\int_{0}^{T} \int_{\omega}\left|\chi b_{\omega} w_{2}^{k}\right|^{2} d x d t
$$

According to (4.2), this gives

$$
\int_{0}^{T} \int_{\omega}\left|b_{\omega} w_{2}^{k}\right|^{2} d x d t \leq \frac{1}{4 K}
$$

for $k$ sufficiently large. This yields a contradiction with (4.3), and concludes the proof of the corollary.

We shall use the following lemma in the proof of Theorem 4.1. A proof is given in Appendix B.2.

Lemma 4.3. For any bicharacteristic curve $\Gamma$ of the d'Alembert operator $P$, there exists a pure sequence $\left(w^{k}\right)_{k \in \mathbb{N}}$ of $\mathscr{C}^{0}\left(0, T ; L^{2}(\Omega)\right) \cap \mathscr{C}^{1}\left(0, T ; H^{-1}(\Omega)\right)$-solutions of P $w^{k}=0$ such that

$$
\left\{\begin{array}{l}
\liminf _{k \rightarrow \infty} E_{0}\left(w^{k}(0)\right) \geq 1 \\
w^{k} \rightarrow 0 \text { in } L^{2}\left(M_{T} ; \mathbb{C}\right)
\end{array}\right.
$$

and the microlocal defect measure of $\left(w^{k}\right)_{k \in \mathbb{N}}$ is supported in $\Gamma$.

We now prove Theorem 4.1 .

Proof of Theorem 4.1. Since $T<T_{\omega \rightarrow \mathcal{O} \rightarrow \omega}$, Definition 2.3 gives the existence of $\rho_{*}=\left(0, x_{*}, \tau_{*}, \eta_{*}\right) \in$ Char $(P) \cap S^{*} M$ such that the following condition holds:

$$
\begin{gathered}
\text { For any } 0<t_{0}<t_{1}<t_{2}<T \text {, we have } \\
\pi\left(\phi_{t_{0}}\left(\rho_{*}\right)\right) \notin(0, T) \times \omega, \quad \text { or } \pi\left(\phi_{t_{1}}\left(\rho_{*}\right)\right) \notin(0, T) \times \mathcal{O}, \quad \text { or } \pi\left(\phi_{t_{2}}\left(\rho_{*}\right)\right) \notin(0, T) \times \omega .
\end{gathered}
$$

We set

$$
\Gamma=\left\{\phi_{s}\left(\rho_{*}\right), s \in[0, T]\right\} .
$$

We now construct the sequence $\left(w_{1}^{k}, w_{2}^{k}\right)_{k \in \mathbb{N}}$.

First, if $\pi(\Gamma) \cap((0, T) \times \omega)=\emptyset$ (which only occurs if $\left.T<T_{\omega}\right)$, we take $w_{1}^{k}=0$ for all $k \in \mathbb{N}$ and $\left(w_{2}^{k}\right)_{k \in \mathbb{N}}$ as given by Lemma 4.3. In this case, (4.2) is clear as $\operatorname{supp}\left(\mu_{2}\right) \subset \Gamma$ and $\pi(\Gamma) \cap((0, T) \times \omega)=\emptyset$ (this is a classical non-observability result for a single wave equation [BLR92, Bur97a, BG97]). 


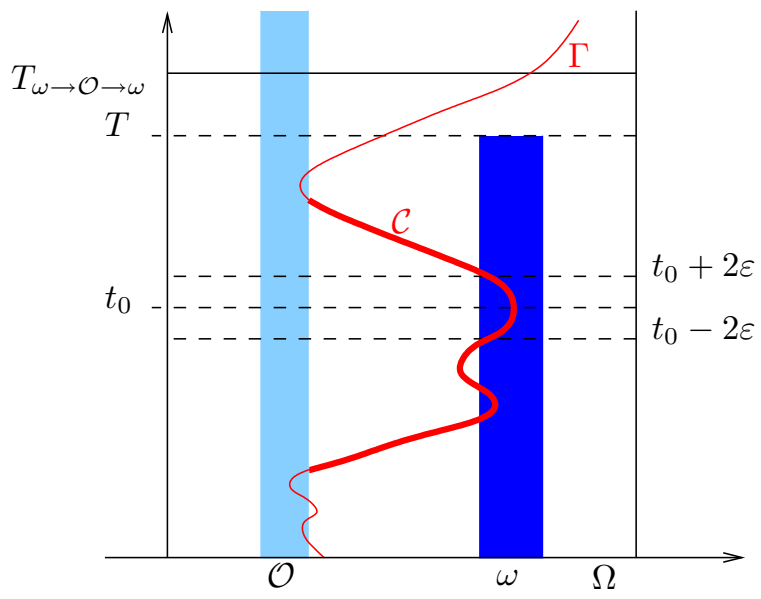

Figure 2: Geometrical situation in the case $T<T_{\omega \rightarrow \mathcal{O} \rightarrow \omega}$ with $\pi(\Gamma) \cap((0, T) \times \omega) \neq \emptyset$.

Second, if $\pi(\Gamma) \cap((0, T) \times \omega) \neq \emptyset$, we choose $\left(w_{1}^{k}\right)_{k \in \mathbb{N}}$ as given by Lemma 4.3. In particular, according to Lemma 4.3, this gives $\liminf _{k \rightarrow \infty}\left(E_{0}\left(w_{1}^{k}(0)\right)+E_{0}\left(w_{2}^{k}(0)\right)\right) \geq 1$ for all $\mathscr{C}^{0}\left(0, T ; L^{2}(\Omega)\right) \cap$ $\mathscr{C}^{1}\left(0, T ; H^{-1}(\Omega)\right)$-sequence $\left(w_{2}^{k}\right)_{k \in \mathbb{N}}$. We also pick some $t_{0} \in(0, T)$ such that $\pi\left(\phi_{t_{0}}\left(\rho_{*}\right)\right) \in(0, T) \times \omega$. The geometrical situation is sketched on Figure 2.

We choose $w_{2}^{k}$ to be the unique (forward and backward) solution of

$$
\begin{cases}P w_{2}^{k}=-B w_{1}^{k} & \text { in }(0, T) \times \Omega \\ \left.\left(w_{2}^{k}, \partial_{t} w_{2}^{k}\right)\right|_{t=t_{0}}=(0,0) & \text { on } \Omega\end{cases}
$$

In particular, the well-posedness of the wave equation yields $w_{2}^{k} \in \mathscr{C}^{0}\left(0, T ; L^{2}(\Omega)\right) \cap \mathscr{C}^{1}\left(0, T ; H^{-1}(\Omega)\right)$ and

$$
\left\|w_{2}^{k}\right\|_{L^{2}\left(M_{T}\right)} \leq C\left\|B w_{1}^{k}\right\|_{L^{2}\left(0, T ; H^{-1}(\Omega)\right)} \leq C\left\|w_{1}^{k}\right\|_{L^{2}\left(M_{T}\right)},
$$

as $B=b(1-\Delta)^{\frac{1}{2}}$. Since $\left(w_{1}^{k}\right)_{k \in \mathbb{N}}$ is bounded in $L^{2}\left(M_{T} ; \mathbb{C}\right)$ from Lemma 4.3 , the sequence $\left(w_{1}^{k}, w_{2}^{k}\right)_{k \in \mathbb{N}}$ is bounded in $L^{2}\left(M_{T} ; \mathbb{C}^{2}\right)$. Moreover, the well-posedness of the wave equation in $\mathscr{C}^{0}\left(0, T ; H^{-1}(\Omega)\right) \cap$ $\mathscr{C}^{1}\left(0, T ; H^{-2}(\Omega)\right)$ also gives

$$
\left\|w_{2}^{k}\right\|_{H^{-1}\left(M_{T}\right)} \leq C\left\|B w_{1}^{k}\right\|_{L^{2}\left(0, T ; H^{-2}(\Omega)\right)} \leq C\left\|w_{1}^{k}\right\|_{H^{-1}\left(M_{T}\right)} \rightarrow 0,
$$

since $w_{1}^{k} \rightarrow 0$ in $L^{2}\left(M_{T} ; \mathbb{C}\right)$. Finally, the sequence $\left(w_{1}^{k}, w_{2}^{k}\right)_{k \in \mathbb{N}}$ satisfies all the conditions (4.1).

Since $\left(w_{1}^{k}, w_{2}^{k}\right) \rightarrow 0$ in $L^{2}\left(M_{T} ; \mathbb{C}^{2}\right)$, and according to [Gér91, Theorem 1], there exists a subsequence (still denoted $\left.\left(W^{k}\right)_{k \in \mathbb{N}}=\left(w_{1}^{k}, w_{2}^{k}\right)_{k \in \mathbb{N}}\right)$ and a microlocal defect measure

$$
\mu=\left(\begin{array}{ll}
\mu_{1} & \mu_{12} \\
\bar{\mu}_{12} & \mu_{2}
\end{array}\right) \in \mathcal{M}_{+}\left(S^{*} M_{T} ; \mathbb{C}^{2 \times 2}\right),
$$

such that for any $\mathcal{A} \in \Psi_{\mathrm{phg}}^{0}\left(M_{T} ; \mathbb{C}^{2 \times 2}\right)$,

$$
\lim _{k \rightarrow \infty}\left(\mathcal{A} W^{k}, W^{k}\right)_{L^{2}\left(M_{T} ; \mathbb{C}^{2}\right)}=\left\langle\mu, \sigma_{0}(\mathcal{A})\right\rangle_{S^{*} M_{T}} .
$$

Moreover, $\mu_{1}$ is the microlocal defect measure associated with the sequence $\left(w_{1}^{k}\right)_{k \in \mathbb{N}}$. As this sequence is (a subsequence of a sequence) choosen by means of Lemma 4.3, we have $\operatorname{supp}\left(\mu_{1}\right) \subset \Gamma$. Observe now that the sequence $\left(W^{k}\right)_{k \in \mathbb{N}}$ satisfies all the assumptions of the first part of Lemma 3.3, which gives $\mu_{2} \in \mathcal{M}_{+}\left(S^{*} M_{T}\right), \operatorname{supp}\left(\mu_{2}\right) \subset \operatorname{Char}(P)$, and $\operatorname{supp}\left(\mu_{12}\right) \subset \operatorname{supp}\left(\mu_{1}\right) \subset \Gamma$. Finally these three measures satisfy the equations

$$
\left\{\begin{array}{l}
\left\langle\mu_{1}, H_{p} a\right\rangle_{S^{*} M_{T}}=0 \\
\left\langle\mu_{2}, H_{p} a\right\rangle_{S^{*} M_{T}}=-\left\langle\operatorname{Im}\left(\mu_{12}\right), 2 b|\eta|_{x} a\right\rangle_{S^{*} M_{T}} \\
\left\langle\operatorname{Im}\left(\mu_{12}\right), H_{p} a\right\rangle_{S^{*} M_{T}}=-\left\langle\mu_{1}, b|\eta|_{x} a\right\rangle_{S^{*} M_{T}}
\end{array}\right.
$$


for any $a \in S_{\mathrm{phg}}^{-1}\left(T^{*} M_{T} ; \mathbb{C}\right)$.

We denote by $\mathcal{C}$ the connected component of $\Gamma \backslash \pi^{-1}((0, T) \times \overline{\mathcal{O}})$ that contains $\Gamma \cap \pi^{-1}((0, T) \times \omega)$ (indicated by a thick line in Figure 2). We have $\phi_{t_{0}}\left(\rho_{*}\right) \in \mathcal{C}$. In the present case, Condition (4.5) gives

$$
\pi(\Gamma) \cap((0, T) \times \omega) \cap((0, T) \times \mathcal{O})=\emptyset .
$$

Moreover, Condition (4.5) yields the uniqueness of such a connected component.

The end of the proof consists in showing that $\operatorname{supp}\left(\mu_{2}\right) \subset \Gamma$ and that $\mathcal{C} \cap \operatorname{supp}\left(\mu_{2}\right)=\emptyset$. For this, we first prove that $\mu_{2}$ vanishes identically on $\left.\pi^{-1}\left(\left(t_{0}-2 \varepsilon, t_{0}+2 \varepsilon\right) \times \Omega\right)\right)$ for $\varepsilon>0$ sufficiently small. Then, a propagation argument with (4.7) gives $\operatorname{supp}\left(\mu_{2}\right) \subset \Gamma$ and that $\mu_{2}$ vanishes on $\mathcal{C}$.

Let us take $\varepsilon>0$ with $2 \varepsilon<\min \left(t_{0}, T-t_{0}\right)$, to be chosen below. The well-posedness for the wave equation (4.6) gives

$$
\begin{aligned}
\left\|w_{2}^{k}\right\|_{L^{2}\left(\left(t_{0}-\varepsilon, t_{0}+\varepsilon\right) \times \Omega\right)} & \leq C\left\|B w_{1}^{k}\right\|_{L^{2}\left(t_{0}-\varepsilon, t_{0}+\varepsilon ; H^{-1}(\Omega)\right)} \\
& \leq C\left\|(1-\Delta)^{-\frac{1}{2}} b(1-\Delta)^{\frac{1}{2}} w_{1}^{k}\right\|_{L^{2}\left(\left(t_{0}-\varepsilon, t_{0}+\varepsilon\right) \times \Omega\right)} \\
& \leq C\left\|\chi(1-\Delta)^{-\frac{1}{2}} b(1-\Delta)^{\frac{1}{2}} w_{1}^{k}\right\|_{L^{2}\left(\left(t_{0}-2 \varepsilon, t_{0}+2 \varepsilon\right) \times \Omega\right)}
\end{aligned}
$$

for some $\chi \in \mathscr{C}_{c}^{\infty}\left(t_{0}-2 \varepsilon, t_{0}+2 \varepsilon\right)$ satisfying $0 \leq \chi \leq 1$ and $\chi=1$ on $\left(t_{0}-\varepsilon, t_{0}+\varepsilon\right)$. In this last expression, we have $\chi(1-\Delta)^{-\frac{1}{2}} b(1-\Delta)^{\frac{1}{2}} \in \Psi_{\text {phg }}^{0}\left(M_{T} ; \mathbb{C}\right)$, with principal symbol $\sigma_{0}\left(\chi(1-\Delta)^{-\frac{1}{2}} b(1-\right.$ $\left.\Delta)^{\frac{1}{2}}\right)=\chi b$. As a consequence, we have

$$
\left\|\chi(1-\Delta)^{-\frac{1}{2}} b(1-\Delta)^{\frac{1}{2}} w_{1}^{k}\right\|_{L^{2}\left(\left(t_{0}-2 \varepsilon, t_{0}+2 \varepsilon\right) \times \Omega\right)}^{2} \rightarrow\left\langle\mu_{1}, \chi^{2} b^{2}\right\rangle_{S^{*} M_{T}}
$$

Since $\pi^{-1}((0, T) \times \omega)$ is an open set in $S^{*} M_{T}$, containing $\phi_{t_{0}}\left(\rho_{*}\right)$, there exists $\varepsilon>0$ such that

$$
\phi_{s}\left(\rho_{*}\right) \in \pi^{-1}((0, T) \times \omega), \quad \text { for all } s \in\left(t_{0}-2 \varepsilon, t_{0}+2 \varepsilon\right) .
$$

For this choice of $\varepsilon$, we have

$\operatorname{supp}\left(\mu_{1}\right) \cap \pi^{-1}\left(\left(t_{0}-2 \varepsilon, t_{0}+2 \varepsilon\right) \times \Omega\right) \subset \Gamma \cap \pi^{-1}\left(\left(t_{0}-2 \varepsilon, t_{0}+2 \varepsilon\right) \times \Omega\right)=\left\{\phi_{s}\left(\rho_{*}\right), s \in\left(t_{0}-2 \varepsilon, t_{0}+2 \varepsilon\right)\right\}$.

According to (4.11), this last set is contained in $\Gamma \cap \pi^{-1}((0, T) \times \omega)$, i.e.

$$
\operatorname{supp}\left(\mu_{1}\right) \cap \pi^{-1}\left(\left(t_{0}-2 \varepsilon, t_{0}+2 \varepsilon\right) \times \Omega\right) \subset \Gamma \cap \pi^{-1}((0, T) \times \omega) .
$$

¿From (4.8), we then obtain $\operatorname{supp}\left(\mu_{1}\right) \cap \pi^{-1}\left(\left(t_{0}-2 \varepsilon, t_{0}+2 \varepsilon\right) \times \Omega\right) \cap \pi^{-1}((0, T) \times \mathcal{O})=\emptyset$, which gives

$$
\operatorname{supp}\left(\mu_{1}\right) \cap \pi^{-1}(\operatorname{supp}(\chi)) \cap \pi^{-1}(\operatorname{supp}(b))=\emptyset .
$$

This, together with (4.10) gives

$$
\left\|\chi(1-\Delta)^{-\frac{1}{2}} b(1-\Delta)^{\frac{1}{2}} w_{1}^{k}\right\|_{L^{2}\left(\left(t_{0}-2 \varepsilon, t_{0}+2 \varepsilon\right) \times \Omega\right)} \rightarrow 0 .
$$

Using (4.9), we now obtain $\left\|w_{2}^{k}\right\|_{L^{2}\left(\left(t_{0}-\varepsilon, t_{0}+\varepsilon\right) \times \Omega\right)} \rightarrow 0$, and thus

$$
\left.\operatorname{supp}\left(\mu_{2}\right) \cap \pi^{-1}\left(\left(t_{0}-\varepsilon, t_{0}+\varepsilon\right) \times \Omega\right)\right)=\emptyset .
$$

As $\operatorname{supp}\left(\mu_{12}\right) \subset \Gamma$, the second equation of (4.7) yields

$$
\left\langle\mu_{2}, H_{p} a\right\rangle_{S^{*} M_{T}}=0
$$

for any $a \in S_{\text {phg }}^{-1}\left(T^{*} M_{T} ; \mathbb{C}\right)$ such that $a=0$ in a neighborhood of $\Gamma$. As in the proof of Proposition 3.2, this gives the invariance of the measure $\mu_{2}$ along the flow $\phi_{s}$ away from $\Gamma$. Together with (4.12), this also yields

$$
\operatorname{supp}\left(\mu_{2}\right) \subset \Gamma .
$$


The second equation of (4.7) also gives (4.13) for any $a \in S_{\text {phg }}^{-1}\left(T^{*} M_{T} ; \mathbb{C}\right)$ such that $a=0$ in a neighborhood of $\pi^{-1}((0, T) \times \mathcal{O})$, as $b$ is supported in $\overline{\mathcal{O}}$. Once again, this gives the invariance of the measure $\mu_{2}$ along the flow $\phi_{s}$ on $S^{*} M_{T} \backslash \pi^{-1}((0, T) \times \overline{\mathcal{O}})$. Together with Equation (4.12), this gives, with a propagation argument,

$$
\operatorname{supp}\left(\mu_{2}\right) \cap \mathcal{C}=\emptyset .
$$

Finally, Equations (4.14) and (4.15) together with the definition of $\mathcal{C}$ give

$$
\operatorname{supp}\left(\mu_{2}\right) \cap \pi^{-1}((0, T) \times \omega)=\emptyset .
$$

This implies (4.2) as $b_{\omega}$ is supported in $\bar{\omega}$, and concludes the proof of Theorem 4.1.

\section{The Hilbert Uniqueness Method and the HUM operator}

\subsection{Controllability and observability for cascade Systems}

In this section, we prove the equivalence between controllability and observability for the systems under view. This result is classical for waves [DR77] and further developed by Lions [Lio88] who coined the name HUM. For completeness, we expose this result in the case of our cascade wave system. To fit also the setting of Section 6 below, where waves with different speeds are studied, we give this equivalence for the following control system with $\gamma>0$ and $\sigma \geq 0$ (see also Section 1.2.3):

$$
\begin{cases}P u_{1}+(1-\Delta)^{\frac{\sigma}{2}} b(x) u_{2}=0 & \text { in }(0, T) \times \Omega, \\ P_{\gamma} u_{2}=b_{\omega}(x) f & \text { in }(0, T) \times \Omega .\end{cases}
$$

Proposition 5.1. Let $s \in \mathbb{R}$. Assume that System (5.1) is well-posed in the space $H^{s-\sigma+1}(\Omega) \times$ $H^{1}(\Omega) \times H^{s-\sigma}(\Omega) \times L^{2}(\Omega)$, that is

$$
\left(u_{1}, u_{2}, \partial_{t} u_{1}, \partial_{t} u_{2}\right) \in \mathscr{C}^{0}\left([0, T] ; H^{s-\sigma+1}(\Omega) \times H^{1}(\Omega) \times H^{s-\sigma}(\Omega) \times L^{2}(\Omega)\right) .
$$

Then, it is controllable in time $T>0$ in this space if and only if the observability inequality

$$
E_{\sigma-s}\left(v_{1}(0)\right)+E_{0}\left(v_{2}(0)\right) \leq C \int_{0}^{T}\left\|b_{\omega} v_{2}\right\|_{L^{2}(\Omega)}^{2} d t
$$

holds for all solutions $\left(v_{1}, v_{2}\right)$ to

$$
\begin{cases}P v_{1}=0 & \text { in }(0, T) \times \Omega, \\ P_{\gamma} v_{2}+b(x)(1-\Delta)^{\frac{\sigma}{2}} v_{1}=0 & \text { in }(0, T) \times \Omega,\end{cases}
$$

assumed to be well-posed in $H^{\sigma-s}(\Omega) \times L^{2}(\Omega) \times H^{\sigma-s-1}(\Omega) \times H^{-1}(\Omega)$, that is

$$
\left(v_{1}, v_{2}, \partial_{t} v_{1}, \partial_{t} v_{2}\right) \in \mathscr{C}^{0}\left([0, T] ; H^{\sigma-s}(\Omega) \times L^{2}(\Omega) \times H^{\sigma-s-1}(\Omega) \times H^{-1}(\Omega)\right) .
$$

Remark 5.2. Note that we have $s=1$ and $\sigma=0$ in the duality between Systems (1.1) and (1.6). We have $s=1$ and $\sigma=1$ in the duality between (2.8) and (2.6).

As a preliminary of the proof we first present some duality framework. According to Definition 1.1, we shall only consider zero initial data: $\left(u_{1}(0), u_{2}(0), \partial_{t} u_{1}(0), \partial_{t} u_{2}(0)\right)=(0,0,0,0)$. Firstly, we suppose that the data $\left(v_{1}(0), v_{2}(0), \partial_{t} v_{1}(0), \partial_{t} v_{2}(0)\right)$ for System (5.3) and the control function $f$ in (5.1) are smooth. Taking the inner product of the first line of (5.1) with $v_{1}$, we obtain

$$
\begin{aligned}
-\left((1-\Delta)^{\frac{\sigma}{2}} b u_{2}, v_{1}\right)_{L^{2}\left(M_{T}\right)} & =\left(P u_{1}, v_{1}\right)_{L^{2}\left(M_{T}\right)} \\
& =\left(u_{1}, P v_{1}\right)_{L^{2}\left(M_{T}\right)}+\left(\partial_{t} u_{1}(T), v_{1}(T)\right)_{L^{2}(\Omega)}-\left(u_{1}(T), \partial_{t} v_{1}(T)\right)_{L^{2}(\Omega)},
\end{aligned}
$$


after an integration by parts. Similarly, taking the inner product of the second line of (1.7) with $v_{2}$, we find

$$
\begin{aligned}
\left(b_{\omega} f, v_{2}\right)_{L^{2}\left(M_{T}\right)} & =\left(P_{\gamma} u_{2}, v_{2}\right)_{L^{2}\left(M_{T}\right)} \\
& =\left(u_{2}, P_{\gamma} v_{2}\right)_{L^{2}\left(M_{T}\right)}+\left(\partial_{t} u_{2}(T), v_{2}(T)\right)_{L^{2}(\Omega)}-\left(u_{2}(T), \partial_{t} v_{2}(T)\right)_{L^{2}(\Omega)} .
\end{aligned}
$$

Summing the last two identities and recalling that $\left(v_{1}, v_{2}\right)$ satisfies $(5.3)$, we obtain the duality identity

$$
\begin{aligned}
\left(f, b_{\omega} v_{2}\right)_{L^{2}\left(M_{T}\right)}= & \left(\partial_{t} u_{1}(T), v_{1}(T)\right)_{L^{2}(\Omega)}-\left(u_{1}(T), \partial_{t} v_{1}(T)\right)_{L^{2}(\Omega)} \\
& +\left(\partial_{t} u_{2}(T), v_{2}(T)\right)_{L^{2}(\Omega)}-\left(u_{2}(T), \partial_{t} v_{2}(T)\right)_{L^{2}(\Omega)}
\end{aligned}
$$

Secondly, using a density argument, together with the well-posedness assumptions, we see that we have

$$
\begin{aligned}
\left(f, b_{\omega} v_{2}\right)_{L^{2}\left(M_{T}\right)}= & \left\langle\partial_{t} u_{1}(T), v_{1}(T)\right\rangle_{H^{s-\sigma}(\Omega), H^{\sigma-s}(\Omega)}-\left\langle u_{1}(T), \partial_{t} v_{1}(T)\right\rangle_{H^{s-\sigma+1}(\Omega), H^{\sigma-s-1}(\Omega)} \\
& +\left(\partial_{t} u_{2}(T), v_{2}(T)\right)_{L^{2}(\Omega)}-\left\langle u_{2}(T), \partial_{t} v_{2}(T)\right\rangle_{H^{1}(\Omega), H^{-1}(\Omega)} .
\end{aligned}
$$

For the proof below we also introduce the following continuous map

$$
\begin{aligned}
S: L^{2}\left(M_{T}\right) & \rightarrow H^{s-\sigma+1}(\Omega) \times H^{1}(\Omega) \times H^{s-\sigma}(\Omega) \times L^{2}(\Omega), \\
f & \left.\mapsto\left(u_{1}, u_{2}, \partial_{t} u_{1}, \partial_{t} u_{2}\right)\right|_{t=T} .
\end{aligned}
$$

where $u=\left(u_{1}, u_{2}\right)$ is the solution to (5.1) with zero initial conditions.

\section{Proof of Proposition 5.1. Controllability $\Rightarrow$ observability.}

We start by assuming controllability, i.e. that $S$ is onto. The open mapping theorem then yields that $S\left(B_{L^{2}\left(M_{T}\right)}(0,1)\right)$ is a neighborhood of $(0,0,0,0)$. Then, for some $\eta>0$ we have

$$
B_{H^{s-\sigma+1} \times H^{1} \times H^{s-\sigma} \times L^{2}}(0, \eta) \subset S\left(B_{L^{2}\left(M_{T}\right)}(0,1)\right),
$$

where $B_{H}(0, r)$ denotes the open ball of radius $r$ centered at 0 in the space $H$. By linearity, this yields

$$
B_{H^{s-\sigma+1} \times H^{1} \times H^{s-\sigma} \times L^{2}}(0,2) \subset S\left(B_{L^{2}\left(M_{T}\right)}\left(0,2 \eta^{-1}\right)\right) .
$$

Now, take $V=\left(v_{1}^{0}, v_{2}^{0}, v_{1}^{1}, v_{2}^{1}\right) \in H^{\sigma-s}(\Omega) \times H^{1}(\Omega) \times H^{\sigma-s-1}(\Omega) \times L^{2}(\Omega)$. With the Riesz representation theorem, we choose $U=\left(u_{1}^{0}, u_{2}^{0}, u_{1}^{1}, u_{2}^{1}\right) \in H^{s-\sigma+1}(\Omega) \times H^{1}(\Omega) \times H^{s-\sigma}(\Omega) \times L^{2}(\Omega)$, such that $\|U\|_{H^{s-\sigma+1}(\Omega) \times H^{1}(\Omega) \times H^{s-\sigma}(\Omega) \times L^{2}(\Omega)}=1$ and

$$
\begin{aligned}
& \left\langle u_{1}^{1}, v_{1}^{0}\right\rangle_{H^{s-\sigma}(\Omega), H^{\sigma-s}(\Omega)}-\left\langle u_{1}^{0}, v_{1}^{1}\right\rangle_{H^{s-\sigma+1}(\Omega), H^{\sigma-s-1}(\Omega)}+\left(u_{2}^{1}, v_{2}^{0}\right)_{L^{2}(\Omega)}-\left\langle u_{2}^{0}, v_{2}^{1}\right\rangle_{H^{1}(\Omega), H^{-1}(\Omega)} \\
& \quad=\|V\|_{H^{\sigma-s}(\Omega) \times H^{1}(\Omega) \times H^{\sigma-s-1}(\Omega) \times L^{2}(\Omega)} .
\end{aligned}
$$

Then, take $f$ such that $S(f)=U$ and $\|f\|_{L^{2}\left(M_{T}\right)} \leq 2 \eta^{-1}$. By (5.4), have

$$
\begin{aligned}
\left(E_{\sigma-s}\left(v_{1}(T)\right)+E_{0}\left(v_{2}(T)\right)\right)^{\frac{1}{2}} & =\|V\|_{H^{\sigma-s}(\Omega) \times H^{1}(\Omega) \times H^{\sigma-s-1}(\Omega) \times L^{2}(\Omega)}=\left(f, b_{\omega} v_{2}\right)_{L^{2}\left(M_{T}\right)} \\
& \leq\|f\|_{L^{2}\left(M_{T}\right)}\left\|b_{\omega} v_{2}\right\|_{L^{2}\left(M_{T}\right)} \leq 2 \eta^{-1}\left\|b_{\omega} v_{2}\right\|_{L^{2}\left(M_{T}\right)},
\end{aligned}
$$

where $\left(v_{1}, v_{2}\right)$ is the backward solution of System (5.3), associated with the final data $\left.\left(v_{1}, v_{2}, \partial_{t} v_{1}, \partial_{t} v_{2}\right)\right|_{t=T}=$ $V$. This yields the observability inequality

$$
E_{\sigma-s}\left(v_{1}(T)\right)+E_{0}\left(v_{2}(T)\right) \leq C\left\|b_{\omega} v_{2}\right\|_{L^{2}\left(M_{T}\right)}^{2} .
$$

for all backward solutions of (5.3). Changing $t$ in $T-t$ in System (5.3) yields Inequality (5.2).

Observability $\Rightarrow$ controllability.

Given $U=\left(u_{1}^{0}, u_{2}^{0}, u_{1}^{1}, u_{2}^{1}\right) \in H^{s-\sigma+1}(\Omega) \times H^{1}(\Omega) \times H^{s-\sigma}(\Omega) \times L^{2}(\Omega)$, we define the following functional

$$
\begin{aligned}
J(V)= & \frac{1}{2}\left\|b_{\omega} v_{2}\right\|_{L^{2}\left(M_{T}\right)}^{2}-\left(\left\langle u_{1}^{1}, v_{1}^{0}\right\rangle_{H^{s-\sigma}(\Omega), H^{\sigma-s}(\Omega)}-\left\langle u_{1}^{0}, v_{1}^{1}\right\rangle_{H^{s-\sigma+1}(\Omega), H^{\sigma-s-1}(\Omega)}\right. \\
& \left.+\left(u_{2}^{1}, v_{2}^{0}\right)_{L^{2}(\Omega)}-\left\langle u_{2}^{0}, v_{2}^{1}\right\rangle_{H^{1}(\Omega), H^{-1}(\Omega)}\right),
\end{aligned}
$$


where $\left(v_{1}, v_{2}\right)(t, x)$ is the backward solution of (5.3) with the data $V=\left(v_{1}^{0}, v_{2}^{0}, v_{1}^{1}, v_{2}^{1}\right)$ at time $t=T$. This quadratic functional is continuous, strictly convex and the observability inequality (5.2) (after having changed $t$ in $T-t$ in System (5.3)) implies that it is coercive. Hence, $J$ admits a unique minimizer $\underline{V}=\left(\underline{v}_{1}^{0}, \underline{v}_{2}^{0}, \underline{v}_{1}^{1}, \underline{v}_{2}^{1}\right)$, satisfying the Euler equation

$$
\begin{gathered}
0=\left(b_{\omega} \underline{v}_{2}, b_{\omega} v_{2}\right)_{L^{2}\left(M_{T}\right)}-\left(\left\langle u_{1}^{1}, v_{1}^{0}\right\rangle_{H^{s-\sigma}(\Omega), H^{\sigma-s}(\Omega)}-\left\langle u_{1}^{0}, v_{1}^{1}\right\rangle_{H^{s-\sigma+1}(\Omega), H^{\sigma-s-1}(\Omega)}\right. \\
\left.+\left(u_{2}^{1}, v_{2}^{0}\right)_{L^{2}(\Omega)}-\left\langle u_{2}^{0}, v_{2}^{1}\right\rangle_{H^{1}(\Omega), H^{-1}(\Omega)}\right) .
\end{gathered}
$$

where $\left(\underline{v}_{1}, \underline{v}_{2}\right)(t, x)$ is the backward solution of (5.3) with the data $\underline{V}$ at time $t=T$. In view of (5.4), this means exactly that $f(t):=b_{\omega} \underline{v}_{2}(t)$ realizes a control for System (5.1).

\subsection{The HUM operator}

Here we use some of the notation and results of the proof of Proposition 5.1 where a control is defined. It is in fact of minimal $L^{2}$ norm. We shall associate an operator to this control.

We set the map

$$
\begin{aligned}
Q: H^{\sigma-s}(\Omega) \times L^{2}(\Omega) \times H^{\sigma-s-1}(\Omega) \times H^{-1}(\Omega) & \rightarrow L^{2}\left(M_{T}\right), \\
\left(v_{1}(T), v_{2}(T), \partial_{t} v_{1}(T), \partial_{t} v_{2}(T)\right) & \mapsto b_{\omega} v_{2},
\end{aligned}
$$

where $v=\left(v_{1}, v_{2}\right)$ is the solution to system (5.3) with final condition $\left(v_{1}(T), v_{2}(T), \partial_{t} v_{1}(T), \partial_{t} v_{2}(T)\right) \in$ $H^{\sigma-s}(\Omega) \times L^{2}(\Omega) \times H^{\sigma-s-1}(\Omega) \times H^{-1}(\Omega)$. We define the natural duality bracket

$$
\begin{aligned}
\left\langle\left(u_{1}^{0}, u_{2}^{0}, u_{1}^{1}, u_{2}^{1}\right),\left(v_{1}^{0}, v_{2}^{0}, v_{1}^{1}, v_{2}^{1}\right)\right\rangle_{*}= & \left\langle u_{1}^{1}, v_{1}^{0}\right\rangle_{H^{s-\sigma}(\Omega), H^{\sigma-s}(\Omega)}-\left\langle u_{1}^{0}, v_{1}^{1}\right\rangle_{H^{s-\sigma+1}(\Omega), H^{\sigma-s-1}(\Omega)} \\
& +\left(u_{2}^{1}, v_{2}^{0}\right)_{L^{2}(\Omega)}-\left\langle u_{2}^{0}, v_{2}^{1}\right\rangle_{H^{1}(\Omega), H^{-1}(\Omega)},
\end{aligned}
$$

between the spaces $H^{s-\sigma+1}(\Omega) \times H^{1}(\Omega) \times H^{s-\sigma}(\Omega) \times L^{2}(\Omega)$ and $H^{\sigma-s}(\Omega) \times L^{2}(\Omega) \times H^{\sigma-s-1}(\Omega) \times$ $H^{-1}(\Omega)$.

From (5.4) we have

$$
\|Q(V)\|_{L^{2}\left(M_{T}\right)}^{2}=\langle S \circ Q(V), V\rangle_{*}, \quad V=\left(v_{1}(T), v_{2}(T), \partial_{t} v_{1}(T), \partial_{t} v_{2}(T)\right) .
$$

We set $\mathcal{L}_{T}=S \circ Q$ (the Gramian operator). If the observability inequality holds, it reads (change $t$ in $T-t)$

$$
\|Q(V)\|_{L^{2}\left(M_{T}\right)} \geq C\|V\|_{H^{\sigma-s}(\Omega) \times H^{1}(\Omega) \times H^{\sigma-s-1}(\Omega) \times L^{2}(\Omega)} .
$$

Then this yields the invertibility of $\mathcal{L}_{T}$ by the Lax-Milgram theorem, which allows one to define the HUM operator:

$$
\mathcal{H}_{T}=\mathcal{L}_{T}^{-1},
$$

that maps continuously $H^{s-\sigma+1}(\Omega) \times H^{1}(\Omega) \times H^{s-\sigma}(\Omega) \times L^{2}(\Omega)$ onto $H^{\sigma-s}(\Omega) \times L^{2}(\Omega) \times H^{\sigma-s-1}(\Omega) \times$ $H^{-1}(\Omega)$.

For a final data $U=\left(u_{1}^{0}, u_{2}^{0}, u_{1}^{1}, u_{2}^{1}\right) \in H^{s-\sigma+1}(\Omega) \times H^{1}(\Omega) \times H^{s-\sigma}(\Omega) \times L^{2}(\Omega)$ we can define $f_{H U M}=Q \circ \mathcal{H}_{T}(U)$ which is a control reaching the target $U$ at final time $T$ for system (5.1):

$$
S f_{H U M}=S \circ Q \circ \mathcal{H}_{T}(U)=S \circ Q \circ(S \circ Q)^{-1}(U)=U .
$$

The identity (5.4) yields that $f_{H U M}$ is precisely the control built in the second part of the proof of Proposition 5.1. The Fenchel-Rockafellar convex-optimization theory [ET74] implies that, actually, $f_{H U M}$ is the unique minimizer of the cost functional $\|f\|_{L^{2}\left(M_{T}\right)}^{2}$ among all controls $f \in L^{2}\left(M_{T}\right)$ for System (5.1). 


\subsection{Microlocal characterization of the HUM operator}

In this section, we develop a precise analysis of the Gramian operator associated to the observation system (2.6). More precisely, we prove that this operator is a matrix of pseudodifferential operators of order zero. We also analyze its ellipticity properties, providing a second proof of Theorem 1.3, together with additional microlocal properties.

Actually, here we follow the program developed by Dehman-Lebeau [DL09], of which the reader can find very nice illustrations in [LN10].

We shall make an intensive use of the Egorov theorem as given in Theorem 2.1. We shall also use smoothing properties of some very particular Fourier integral operators; these results are collected in Appendix A.

\subsubsection{A simplified model: a system of coupled half-wave equations}

In this section, we consider the control problem for two coupled half-wave equations

$$
\begin{cases}\left(\partial_{t}-i \lambda\right) u_{1}-\frac{1}{2 i} b u_{2}=0 & \text { in }(0, T) \times \Omega \\ \left(\partial_{t}-i \lambda\right) u_{2}=b_{\omega} f & \text { in }(0, T) \times \Omega .\end{cases}
$$

This system is much simpler than System (1.1). Yet, this section will help the reader to understand the key aspects of the microlocal characterization of the HUM operator, without the additional technical difficulties that one faces when addressing the full wave system (1.1). That analysis is postponed to the next section. Note that the coefficient $\frac{-1}{2 i}$ is chosen here to fit the setting of System (1.1) (see Section 5.3.2 below) and has no importance here.

The first-order system $(5.7)$ is well-posed for initial data $\left(u_{1}(0), u_{2}(0)\right) \in H^{s}\left(\Omega ; \mathbb{C}^{2}\right)$ and a right hand-side $f \in L^{1}\left(\mathbb{R} ; H^{s}(\Omega)\right)$, giving rise to a unique solution $\left(u_{1}, u_{2}\right) \in \mathscr{C}^{0}\left(\mathbb{R} ; H^{s}\left(\Omega ; \mathbb{C}^{2}\right)\right) \cap$ $\mathscr{C}^{1}\left(\mathbb{R} ; H^{s-1}\left(\Omega ; \mathbb{C}^{2}\right)\right.$ ) (see for instance [Hör85, Chapter 23.1]). Note that there is here no gain of regularity in the state space (as opposed to the full wave-system (1.1)). The associated observation problem is the following

$$
\begin{cases}\left(\partial_{t}-i \lambda\right) v_{1}=0 & \text { in }(0, T) \times \Omega \\ \left(\partial_{t}-i \lambda\right) v_{2}+\frac{1}{2 i} b v_{1}=0 & \text { in }(0, T) \times \Omega \\ \left(v_{1}(0), v_{1}(0)\right)=(g, h) \in L^{2}\left(\Omega ; \mathbb{C}^{2}\right) & \end{cases}
$$

together with the observability inequality

$$
\|g\|_{L^{2}(\Omega)}^{2}+\|h\|_{L^{2}(\Omega)}^{2} \leq C \int_{0}^{T}\left\|b_{\omega} v_{2}\right\|_{L^{2}(\Omega)}^{2} d t .
$$

In this setting, similarly to what is done in Section 5.1, the controllability of System (5.7) is equivalent to estimate $(5.9)$ for all $(g, h) \in L^{2}\left(\Omega ; \mathbb{C}^{2}\right)$ and $\left(v_{1}, v_{2}\right)$ associated solutions of $(5.8)$.

We recall that the flow $\left(\varphi_{t}^{+}\right)_{t \in \mathbb{R}}$, used in the statement of Theorem 5.3 is defined by (2.5).

Theorem 5.3. We have

$$
\int_{0}^{T}\left\|b_{\omega} v_{2}\right\|_{L^{2}(\Omega)}^{2} d t=\left(\mathcal{G}_{T}^{+}(g, h),(g, h)\right)_{L^{2}\left(\Omega ; \mathbb{C}^{2}\right)} .
$$

where $\mathcal{G}_{T}^{+} \in \mathcal{L}\left(L^{2}\left(\Omega ; \mathbb{C}^{2}\right)\right)$ is the Gramian operator of $(5.7)$.

Moreover, there exists $G_{T}^{+} \in \Psi_{\mathrm{phg}}^{0}\left(\Omega ; \mathbb{C}^{2 \times 2}\right)$, and $R_{T}$ an infinitely smoothing operator on $\Omega$ such that

$$
\mathcal{G}_{T}^{+}=G_{T}^{+}+R_{T}
$$

where the principal symbol of $G_{T}^{+}$is

$\sigma_{0}\left(G_{T}^{+}\right)=\left(\begin{array}{cc}\frac{1}{4} \int_{0}^{T} b_{\omega}^{2} \circ \varphi_{t}^{+}\left(\int_{0}^{t} b \circ \varphi_{\sigma}^{+} d \sigma\right)^{2} d t & \frac{1}{2 i} \int_{0}^{T} b_{\omega}^{2} \circ \varphi_{t}^{+}\left(\int_{0}^{t} b \circ \varphi_{\sigma}^{+} d \sigma\right) d t \\ -\frac{1}{2 i} \int_{0}^{T} b_{\omega}^{2} \circ \varphi_{t}^{+}\left(\int_{0}^{t} b \circ \varphi_{\sigma}^{+} d \sigma\right) d t & \int_{0}^{T} b_{\omega}^{2} \circ \varphi_{t}^{+} d t\end{array}\right) \in S_{\mathrm{phg}}^{0}\left(T^{*} \Omega, \mathbb{C}^{2 \times 2}\right)$. 
In particular, we have

$$
\operatorname{det}\left(\sigma_{0}\left(G_{T}^{+}\right)\right)=\frac{1}{8} \int_{0}^{T} \int_{0}^{T}\left(b_{\omega}^{2} \circ \varphi_{t_{1}}^{+}\right)\left(b_{\omega}^{2} \circ \varphi_{t_{2}}^{+}\right)\left(\int_{t_{1}}^{t_{2}} b \circ \varphi_{\sigma}^{+} d \sigma\right)^{2} d t_{1} d t_{2} \in S_{\mathrm{phg}}^{0}\left(T^{*} \Omega\right) .
$$

The next corollary both proves the observability of (5.8) and characterizes the HUM operator. The connection between the HUM operator $\left(\mathcal{G}_{T}^{+}\right)^{-1}$ and the construction of the control of minimal $L^{2}$-norm for the present simplified half-wave model can be done as in Sections 5.1-5.2. This is left to the reader. Recall that the time $T_{\omega \rightarrow \mathcal{O} \rightarrow \omega}^{+}$is defined in Definition 2.4.

Corollary 5.4. Assume that both $\omega$ and $\mathcal{O}$ satisfy $G C C$ and that $T>T_{\omega \rightarrow \mathcal{O} \rightarrow \omega}^{+}$. Then, we have the following properties:

1. The operator $G_{T}^{+} \in \Psi_{\mathrm{phg}}^{0}\left(\Omega, \mathbb{C}^{2 \times 2}\right)$ is elliptic.

2. The operator $\mathcal{G}_{T}^{+}$is coercive on $L^{2}\left(\Omega ; \mathbb{C}^{2}\right)$ :

$$
\int_{0}^{T}\left\|b_{\omega} v_{2}\right\|_{L^{2}(\Omega)}^{2} d t=\left(\mathcal{G}_{T}^{+}(g, h),(g, h)\right)_{L^{2}\left(\Omega ; \mathbb{C}^{2}\right)} \geq C\|(g, h)\|_{L^{2}\left(\Omega ; \mathbb{C}^{2}\right)}^{2},
$$

for all $(g, h) \in L^{2}\left(\Omega ; \mathbb{C}^{2}\right)$ and $\left(v_{1}, v_{2}\right)$ associated solutions of $(5.8)$.

3. The operator $\mathcal{G}_{T}^{+}$is invertible in $\mathcal{L}\left(L^{2}(\Omega)\right)$. Its inverse $\left(\mathcal{G}_{T}^{+}\right)^{-1}$, the HUM operator, can be decomposed as $\left(\mathcal{G}_{T}^{+}\right)^{-1}=\Lambda_{T}^{+}+R_{T}$ where $R_{T} \in \mathcal{R}^{\infty}(\Omega)$ and $\Lambda_{T}^{+} \in S_{\mathrm{phg}}^{0}\left(T^{*} \Omega, \mathbb{C}^{2 \times 2}\right)$, with principal symbol

$$
\sigma_{0}\left(\Lambda_{T}^{+}\right)=\operatorname{det}\left(\sigma_{0}\left(G_{T}^{+}\right)\right)^{-1}\left(\begin{array}{cl}
\int_{0}^{T} b_{\omega}^{2} \circ \varphi_{t}^{+} d t & \frac{1}{2 i} \int_{0}^{T} b_{\omega}^{2} \circ \varphi_{t}^{+}\left(\int_{0}^{t} b \circ \varphi_{\sigma}^{+} d \sigma\right) d t \\
-\frac{1}{2 i} \int_{0}^{T} b_{\omega}^{2} \circ \varphi_{t}^{+}\left(\int_{0}^{t} b \circ \varphi_{\sigma}^{+} d \sigma\right) d t & \frac{1}{4} \int_{0}^{T} b_{\omega}^{2} \circ \varphi_{t}^{+}\left(\int_{0}^{t} b \circ \varphi_{\sigma}^{+} d \sigma\right)^{2} d t
\end{array}\right) .
$$

4. In particular, the HUM operator $\left(\mathcal{G}_{T}^{+}\right)^{-1}$ is an isomorphism of $H^{s}\left(\Omega ; \mathbb{C}^{2}\right)$ for all $s \geq 0$ and we have

$$
\mathrm{WF}^{s}\left(\left(\mathcal{G}_{T}^{+}\right)^{-1}(f, g)\right)=\mathrm{WF}^{s}(f, g) .
$$

Recall that the notation $\mathcal{R}^{\infty}$ was introduced in Definition 2.2. The definition of $\mathrm{WF}^{s}(u)$, for $u \in \mathscr{D}^{\prime}(\Omega)$ is for instance given in [Ler10, Definition 1.2.21]. The wavefront set of a couple, or, more generally a $k$-tuple $\left(f_{1}, f_{2}, \ldots, f_{k}\right) \in \mathscr{D}^{\prime}\left(\Omega ; \mathbb{C}^{k}\right)$, is defined by (see for instance [Den82]).

$$
\mathrm{WF}^{s}\left(f_{1}, f_{2}, \ldots, f_{k}\right)=\bigcup_{j=1}^{k} \mathrm{WF}^{s}\left(f_{j}\right)
$$

Corollary 5.4 is proved at the end of this section.

Proof of Theorem 5.3. Let us recall that the group $e^{i t \lambda}$ is defined at the end of Section 2.1. The Duhamel formula in (5.8) gives the explicit representations

$$
\begin{gathered}
v_{1}(t)=e^{i t \lambda} \Pi_{+} g+\Pi_{0} g \\
v_{2}(t)=e^{i t \lambda} \Pi_{+} h+\Pi_{0} h-\frac{1}{2 i} \int_{0}^{t}\left(e^{i(t-\sigma) \lambda} \Pi_{+} b v_{1}(\sigma)+\Pi_{0} b v_{1}(\sigma)\right) d \sigma .
\end{gathered}
$$

Developping this last expression, we have in particular,

$$
v_{2}(t)=e^{i t \lambda} \Pi_{+} h-\frac{1}{2 i} \int_{0}^{t} e^{i(t-\sigma) \lambda} \Pi_{+} b e^{i \sigma \lambda} \Pi_{+} g d \sigma+R_{t}(g, h),
$$

where

$$
R_{t}(g, h)=\Pi_{0} h-\frac{1}{2 i} t \Pi_{0} b \Pi_{0} g+\frac{1}{2} \lambda^{-1}\left(e^{i t \lambda}-1\right) \Pi_{+} b \Pi_{0} g+\frac{1}{2} \Pi_{0} b \lambda^{-1}\left(e^{i t \lambda}-1\right) \Pi_{+} g .
$$


Recall that $\lambda^{-1} \in \mathcal{L}\left(H_{+}^{s}(\Omega) ; H_{+}^{s+1}(\Omega)\right)$ is defined at the end of Section 2.1.

Hence, $R_{t}$ is a continuous family of infinitely smoothing operators, since $\Pi_{0} \in \mathcal{L}\left(H^{s}(\Omega) ; \mathbb{C}\right)$ for all $s \in \mathbb{R}$ and $b$ and $e^{i t \lambda}$ preserve the regularity.

Now, let us compute the observation

$$
\int_{0}^{T}\left\|b_{\omega} v_{2}\right\|_{L^{2}(\Omega)}^{2} d t=\int_{0}^{T}\left\|b_{\omega} e^{i t \lambda} \Pi_{+} h-\frac{1}{2 i} b_{\omega} e^{i t \lambda} \Pi_{+} \int_{0}^{t} e^{-i \sigma \lambda} b e^{i \sigma \lambda} d \sigma \Pi_{+} g+R_{t}(g, h)\right\|_{L^{2}(\Omega)}^{2} d t .
$$

According to the Egorov theorem (see Theorem 2.1), for any $N \in \mathbb{N}$, we have $e^{-i \sigma \lambda} b e^{i \sigma \lambda}-R_{t}^{N} \in$ $\mathscr{C}^{0}\left(\mathbb{R}, \Psi_{\text {phg }}^{0}(\Omega)\right)$, with principal symbol $b \circ \varphi_{\sigma}^{+}(x, \eta)$, where $R_{t}^{N}$ is a continuous family of $N$-smoothing operators. Hence, the operator

$$
B_{t}^{+}:=\int_{0}^{t} e^{-i \sigma \lambda} b e^{i \sigma \lambda} d \sigma
$$

is in $\mathscr{C}^{0}\left(\mathbb{R}, \Psi_{\mathrm{phg}}^{0}(\Omega)\right)$ up to a continuous family of $N$-smoothing operators, and we have

$$
\sigma_{0}\left(B_{t}^{+}\right)(x, \eta)=\int_{0}^{t} b \circ \varphi_{\sigma}^{+}(x, \eta) d \sigma, \quad(x, \eta) \in T^{*} \Omega .
$$

Coming back to (5.12) and developping the inner product, we obtain

$$
\begin{aligned}
\int_{0}^{T}\left\|b_{\omega} v_{2}\right\|_{L^{2}(\Omega)}^{2} d t= & \int_{0}^{T}\left(\Pi_{+} e^{-i t \lambda} b_{\omega}^{2} e^{i t \lambda} \Pi_{+} h, h\right)_{L^{2}(\Omega)} d t-\frac{1}{2 i} \int_{0}^{T}\left(\Pi_{+} e^{-i t \lambda} b_{\omega}^{2} e^{i t \lambda} \Pi_{+} B_{t}^{+} \Pi_{+} g, h\right)_{L^{2}(\Omega)} d t \\
& +\frac{1}{2 i} \int_{0}^{T}\left(\Pi_{+}\left(B_{t}^{+}\right)^{*} \Pi_{+} e^{-i t \lambda} b_{\omega}^{2} e^{i t \lambda} \Pi_{+} h, g\right)_{L^{2}(\Omega)} d t \\
& +\frac{1}{4} \int_{0}^{T}\left(\Pi_{+}\left(B_{t}^{+}\right)^{*} \Pi_{+} e^{-i t \lambda} b_{\omega}^{2} e^{i t \lambda} \Pi_{+} B_{t}^{+} \Pi_{+} g, g\right)_{L^{2}(\Omega)} d t+\int_{0}^{T}\left(\tilde{R}_{t}(g, h),(g, h)\right)_{L^{2}(\Omega)} d t
\end{aligned}
$$

where $\tilde{R}_{t}$ is a continuous family of $N$-smoothing operators.

Setting

$$
G_{T}^{+}=\left(\begin{array}{cc}
\frac{1}{4} \int_{0}^{T} \Pi_{+}\left(B_{t}^{+}\right)^{*} \Pi_{+} e^{-i t \lambda} b_{\omega}^{2} e^{i t \lambda} \Pi_{+} B_{t}^{+} \Pi_{+} d t & +\frac{1}{2 i} \int_{0}^{T} \Pi_{+}\left(B_{t}^{+}\right)^{*} \Pi_{+} e^{-i t \lambda} b_{\omega}^{2} e^{i t \lambda} \Pi_{+} d t \\
-\frac{1}{2 i} \int_{0}^{T} \Pi_{+} e^{-i t \lambda} b_{\omega}^{2} e^{i t \lambda} \Pi_{+} B_{t}^{+} \Pi_{+} d t & \int_{0}^{T} \Pi_{+} e^{-i t \lambda} b_{\omega}^{2} e^{i t \lambda} \Pi_{+} d t
\end{array}\right),
$$

we find $G_{T}^{+} \in \Psi_{\mathrm{phg}}^{0}\left(\Omega, \mathbb{C}^{2 \times 2}\right)$, since, according to the Egorov theorem, $e^{-i t \lambda} b_{\omega}^{2} e^{i t \lambda} \in \Psi_{\mathrm{phg}}^{0}(\Omega)$ with principal symbol $b_{\omega}^{2} \circ \varphi_{t}^{+}(x, \eta)$. The pseudodifferential calculus directly yields the principal symbol

$\sigma_{0}\left(G_{T}^{+}\right)=\left(\begin{array}{cc}\frac{1}{4} \int_{0}^{T} b_{\omega}^{2} \circ \varphi_{t}^{+}\left(\int_{0}^{t} b \circ \varphi_{\sigma}^{+} d \sigma\right)^{2} d t & \frac{1}{2 i} \int_{0}^{T} b_{\omega}^{2} \circ \varphi_{t}^{+}\left(\int_{0}^{t} b \circ \varphi_{\sigma}^{+} d \sigma\right) d t \\ -\frac{1}{2 i} \int_{0}^{T} b_{\omega}^{2} \circ \varphi_{t}^{+}\left(\int_{0}^{t} b \circ \varphi_{\sigma}^{+} d \sigma\right) d t & \int_{0}^{T} b_{\omega}^{2} \circ \varphi_{t}^{+} d t\end{array}\right) \in S_{\mathrm{phg}}^{0}\left(T^{*} \Omega, \mathbb{C}^{2 \times 2}\right)$

We have thus obtained

$$
\int_{0}^{T}\left\|b_{\omega} v_{2}\right\|_{L^{2}(\Omega)}^{2} d t=\left(\left(G_{T}^{+}+R_{T}\right)(g, h),(g, h)\right)_{L^{2}\left(\Omega ; \mathbb{C}^{2}\right)},
$$

where $R_{T}$ is an infinitely smoothing operator.

Computing $\operatorname{det}\left(\sigma_{0}\left(G_{T}^{+}\right)\right)$, we find

$$
\begin{aligned}
\operatorname{det}\left(\sigma_{0}\left(G_{T}^{+}\right)\right)= & \frac{1}{4}\left(\int_{0}^{T} b_{\omega}^{2} \circ \varphi_{t}^{+} d t\right)\left(\int_{0}^{T} b_{\omega}^{2} \circ \varphi_{t}^{+}\left(\int_{0}^{t} b \circ \varphi_{\sigma}^{+} d \sigma\right)^{2} d t\right) \\
& -\frac{1}{4}\left(\int_{0}^{T} b_{\omega}^{2} \circ \varphi_{t}^{+}\left(\int_{0}^{t} b \circ \varphi_{\sigma}^{+} d \sigma\right) d t\right)^{2} \\
= & \frac{1}{4} \int_{0}^{T} \int_{0}^{T}\left(b_{\omega}^{2} \circ \varphi_{t_{1}}^{+}\right)\left(b_{\omega}^{2} \circ \varphi_{t_{2}}^{+}\right)\left(\int_{0}^{t_{2}} b \circ \varphi_{\sigma}^{+} d \sigma\right)^{2} d t_{1} d t_{2} \\
& -\frac{1}{4} \int_{0}^{T} \int_{0}^{T}\left(b_{\omega}^{2} \circ \varphi_{t_{1}}^{+}\right)\left(b_{\omega}^{2} \circ \varphi_{t_{2}}^{+}\right)\left(\int_{0}^{t_{1}} b \circ \varphi_{\sigma}^{+} d \sigma\right)\left(\int_{0}^{t_{2}} b \circ \varphi_{\sigma}^{+} d \sigma\right) d t_{1} d t_{2} .
\end{aligned}
$$


Given a function $f\left(t_{1}, t_{2}\right)$ defined on the square $[0, T]^{2}$, we notice that its mean value is equal to that of its symmetric part with respect to the diagonal:

$$
\int_{0}^{T} \int_{0}^{T} f\left(t_{1}, t_{2}\right) d t_{1} d t_{2}=\int_{0}^{T} \int_{0}^{T} \frac{1}{2}\left(f\left(t_{1}, t_{2}\right)+f\left(t_{2}, t_{1}\right)\right) d t_{1} d t_{2} .
$$

Applying this remark to the function $\left(t_{1}, t_{2}\right) \mapsto\left(b_{\omega}^{2} \circ \varphi_{t_{1}}^{+}\right)\left(b_{\omega}^{2} \circ \varphi_{t_{2}}^{+}\right)\left(\int_{0}^{t_{2}} b \circ \varphi_{\sigma}^{+} d \sigma\right)^{2}$, we obtain

$$
\begin{aligned}
4 \operatorname{det}\left(\sigma_{0}\left(G_{T}^{+}\right)\right)= & \int_{0}^{T} \int_{0}^{T}\left(b_{\omega}^{2} \circ \varphi_{t_{1}}^{+}\right)\left(b_{\omega}^{2} \circ \varphi_{t_{2}}^{+}\right) \frac{1}{2}\left[\left(\int_{0}^{t_{1}} b \circ \varphi_{\sigma}^{+} d \sigma\right)^{2}+\left(\int_{0}^{t_{2}} b \circ \varphi_{\sigma}^{+} d \sigma\right)^{2}\right] d t_{1} d t_{2} \\
& -\int_{0}^{T} \int_{0}^{T}\left(b_{\omega}^{2} \circ \varphi_{t_{1}}^{+}\right)\left(b_{\omega}^{2} \circ \varphi_{t_{2}}^{+}\right)\left(\int_{0}^{t_{1}} b \circ \varphi_{\sigma}^{+} d \sigma\right)\left(\int_{0}^{t_{2}} b \circ \varphi_{\sigma}^{+} d \sigma\right) d t_{1} d t_{2} \\
= & \frac{1}{2} \int_{0}^{T} \int_{0}^{T}\left(b_{\omega}^{2} \circ \varphi_{t_{1}}^{+}\right)\left(b_{\omega}^{2} \circ \varphi_{t_{2}}^{+}\right)\left(\int_{t_{1}}^{t_{2}} b \circ \varphi_{\sigma}^{+} d \sigma\right)^{2} d t_{1} d t_{2} .
\end{aligned}
$$

This concludes the proof of Theorem 5.3.

Proof of Corollary 5.4. Let us first prove Item 1. Take $\rho=(x, \eta) \in S^{*} \Omega$. Since $T>T_{\omega \rightarrow \mathcal{O} \rightarrow \omega}^{+}$, there exists $0<t_{1}(\rho)<t_{1,2}(\rho)<t_{2}(\rho)<T$ such that

$$
\pi^{-1}\left(\varphi_{t_{1}(\rho)}^{+}(\rho)\right) \in \omega, \quad \pi^{-1}\left(\varphi_{t_{1,2}(\rho)}^{+}(\rho)\right) \in \mathcal{O}, \quad \pi^{-1}\left(\varphi_{t_{2}(\rho)}^{+}(\rho)\right) \in \omega .
$$

Since the functions $b$ and $b_{\omega}$ are continuous this yields

$$
\operatorname{det}\left(\sigma_{0}\left(G_{T}^{+}\right)\right)(\rho)=\frac{1}{8} \int_{0}^{T} \int_{0}^{T}\left(b_{\omega}^{2} \circ \varphi_{t_{1}}^{+}(\rho)\right)\left(b_{\omega}^{2} \circ \varphi_{t_{2}}^{+}(\rho)\right)\left(\int_{t_{1}}^{t_{2}} b \circ \varphi_{\sigma}^{+}(\rho) d \sigma\right)^{2} d t_{1} d t_{2}>0 .
$$

The manifold $S^{*} \Omega$ being compact, we have $\operatorname{det}\left(\sigma_{0}\left(G_{T}^{+}\right)\right) \geq C>0$ on $S^{*} \Omega$. The matrix $\sigma_{0}\left(G_{T}^{+}\right)$has a non-negative trace and positive determinant, and is hence positive definite. Hence, $G_{T}^{+}$is elliptic.

The proof of Item 2 consists in two steps (using a compactness-uniqueness argument as in Section 3 for the proof of Theorem 1.3). The first one is the following high-frequency observability estimate:

$$
\int_{0}^{T}\left\|b_{\omega} v_{2}\right\|_{L^{2}(\Omega)}^{2} d t=\left(\mathcal{G}_{T}^{+}(g, h),(g, h)\right)_{L^{2}\left(\Omega ; \mathbb{C}^{2}\right)} \geq C\|(g, h)\|_{L^{2}\left(\Omega ; \mathbb{C}^{2}\right)}^{2}-C^{\prime}\|(g, h)\|_{H^{-1}\left(\Omega ; \mathbb{C}^{2}\right)}^{2},
$$

for all $(g, h) \in L^{2}\left(\Omega ; \mathbb{C}^{2}\right)$ and $\left(v_{1}, v_{2}\right)$ associated solutions of $(5.8)$.

Using the Gårding inequality for the operator $G_{T}^{+} \in \Psi_{\mathrm{phg}}^{0}(\Omega)$ (see for instance [Tay81, Chapter 2] or [CP82, Chapter 4]), this gives the existence of $C, C^{\prime}>0$ such that, for all $(g, h) \in L^{2}\left(\Omega ; \mathbb{C}^{2}\right)$,

$$
\left(G_{T}^{+}(g, h),(g, h)\right)_{L^{2}\left(\Omega ; \mathbb{C}^{2}\right)} \geq C\|(g, h)\|_{L^{2}\left(\Omega ; \mathbb{C}^{2}\right)}^{2}-C^{\prime}\|(g, h)\|_{H^{-1}\left(\Omega ; \mathbb{C}^{2}\right)}^{2} .
$$

Recalling that $R_{T}$ is 1 -smoothing, i.e. in particular $R_{T} \in \mathcal{L}\left(H^{-1}\left(\Omega ; \mathbb{C}^{2}\right) ; L^{2}\left(\Omega ; \mathbb{C}^{2}\right)\right)$, we have for all $\varepsilon>0$,

$$
\left|\left(R_{T}(g, h),(g, h)\right)_{L^{2}\left(\Omega ; \mathbb{C}^{2}\right)}\right| \leq \frac{C^{\prime \prime}}{\varepsilon}\|(g, h)\|_{H^{-1}\left(\Omega ; \mathbb{C}^{2}\right)}^{2}+\varepsilon\|(g, h)\|_{L^{2}\left(\Omega ; \mathbb{C}^{2}\right)}^{2},
$$

and hence

$$
\begin{aligned}
\left(\mathcal{G}_{T}^{+}(g, h),(g, h)\right)_{L^{2}\left(\Omega ; \mathbb{C}^{2}\right)} & =\left(G_{T}^{+}(g, h),(g, h)\right)_{L^{2}\left(\Omega ; \mathbb{C}^{2}\right)}+\left(R_{T}(g, h),(g, h)\right)_{L^{2}\left(\Omega ; \mathbb{C}^{2}\right)} \\
& \geq(C-\varepsilon)\|(g, h)\|_{L^{2}\left(\Omega ; \mathbb{C}^{2}\right)}^{2}-\left(C^{\prime}+\frac{C^{\prime \prime}}{\varepsilon}\right)\|(g, h)\|_{H^{-1}\left(\Omega ; \mathbb{C}^{2}\right)}^{2} .
\end{aligned}
$$

Taking $\varepsilon$ sufficiently small concludes the proof of (5.14).

The second step of the proof of Item 2 follows Section 3.2. We consider

$$
\begin{gathered}
\mathcal{N}(T)=\left\{(g, h) \in L^{2}\left(\Omega ; \mathbb{C}^{2}\right) \text { such that the associated solution of }(5.8)\right. \\
\text { satisfies } \left.v_{2}(t, x)=0 \text { for all }(t, x) \in(0, T) \times \omega\right\}
\end{gathered}
$$


and prove that $\mathcal{N}(T)=\{0\}$ for $T>T_{\omega \rightarrow \mathcal{O} \rightarrow \omega}^{+}$. Indeed, a proof similar to that of Lemma 3.4 yields that $\mathcal{N}(T)$ is a finite dimensional subspace of $H^{1}\left(\Omega ; \mathbb{C}^{2}\right)$, stable by the action of the operator

$$
\left(\begin{array}{cc}
-i \lambda & 0 \\
\frac{1}{2 i} b & -i \lambda
\end{array}\right)
$$

Hence, it contains an eigenfunction of this operator, $\left(\varphi_{1}, \varphi_{2}\right)$ :

$$
\left\{\begin{array}{l}
-i \lambda \varphi_{1}=\mu \varphi_{1} \\
\frac{b}{2 i} \varphi_{1}-i \lambda \varphi_{2}=\mu \varphi_{2}
\end{array}\right.
$$

In particular, $\mu$ is then an eigenvalue of the antiadjoint operator $-i \lambda$ and thus $\mu \in i \mathbb{R}$. Taking the $L^{2}(\Omega)$-inner product of the first line of (5.15) with $\varphi_{2}$, and that of the second line of (5.15) with $\varphi_{1}$, we obtain

$$
\left\{\begin{array}{l}
i\left(\lambda \varphi_{2}, \varphi_{1}\right)_{L^{2}(\Omega)}=-\mu\left(\varphi_{2}, \varphi_{1}\right)_{L^{2}(\Omega)} \\
\frac{b}{2 i}\left(\varphi_{1}, \varphi_{1}\right)_{L^{2}(\Omega)}-i\left(\lambda \varphi_{2}, \varphi_{1}\right)_{L^{2}(\Omega)}=\mu\left(\varphi_{2}, \varphi_{1}\right)_{L^{2}(\Omega)}
\end{array}\right.
$$

Adding these two lines yields

$$
\left(b \varphi_{1}, \varphi_{1}\right)_{L^{2}(\Omega)}=0 .
$$

Since $b \geq 0$ and $b$ does not vanish identically, this proves that $\varphi_{1}=0$ on $\mathcal{O}$. As the first equation of (5.15) gives $-\Delta \varphi_{1}=\mu^{2} \varphi_{1}, \varphi_{1}$ is an eigenfunction of the Laplace operator vanishing on $\mathcal{O}$, a unique continuation result (see for instance the classical reference [Aro57, AKS62], the book [Zui83] or the exposition article [LL11]) yields $\varphi_{1}=0$ on $\Omega$.

Moreover, $(g, h) \in \mathcal{N}(T)$ yields $\varphi_{2}=0$ on $\omega$. This proves that $\varphi_{2}=0$ on $\Omega$, as $\varphi_{2}$ is then also an eigenfunction of the Laplace operator. This is similar to Remark 3.5. We then obtain $\mathcal{N}(T)=\{0\}$.

Inequality (5.14) is the analogue to the weak observability inequality of Proposition (3.2). Using the same contradiction argument as in Section 3.2 concludes the proof of the observability inequality (5.10).

The proof of Item 3 is inspired by [DL09, Theorem 4.1]. First, $\mathcal{G}_{T}^{+}$is a bounded selfadjoint and coercive operator and is hence invertible according to the Riesz Theorem. Second, since $G_{T}^{+} \in$ $\Psi_{\text {phg }}^{0}\left(\Omega, \mathbb{C}^{2 \times 2}\right)$ is elliptic, there exists (see for instance [Hör85, Theorem 18.1.24]) a parametrix $\Lambda_{T}^{+} \in$ $\Psi_{\text {phg }}^{0}\left(\Omega, \mathbb{C}^{2 \times 2}\right)$ such that

$$
\Lambda_{T}^{+} G_{T}^{+}=\mathrm{Id}+R, \quad \text { with } \quad R \in \Psi_{\mathrm{phg}}^{-\infty}\left(\Omega, \mathbb{C}^{2 \times 2}\right) \quad \text { and } \quad \sigma_{0}\left(\Lambda_{T}^{+}\right)=\sigma_{0}\left(G_{T}^{+}\right)^{-1} .
$$

Hence, using the decomposition $\mathcal{G}_{T}^{+}=G_{T}^{+}+R_{T}$, with $R_{T}$ infinitely smoothing, we have

$$
\Lambda_{T}^{+} \mathcal{G}_{T}^{+}=\Lambda_{T}^{+}\left(G_{T}^{+}+R_{T}\right)=\operatorname{Id}+R+\Lambda_{T}^{+} R_{T},
$$

where $\Lambda_{T}^{+} R_{T}$ is infinitely smoothing. Applying the operator $\left(\mathcal{G}_{T}^{+}\right)^{-1} \in \mathcal{L}\left(L^{2}(\Omega)\right)$ to this identity, we obtain

$$
\left(\mathcal{G}_{T}^{+}\right)^{-1}=\Lambda_{T}^{+}-\left(R+\Lambda_{T}^{+} R_{T}\right)\left(\mathcal{G}_{T}^{+}\right)^{-1} .
$$

Observing that the operator $\left(R+\Lambda_{T}^{+} R_{T}\right)\left(\mathcal{G}_{T}^{+}\right)^{-1}$ is in $\mathcal{R}^{\infty}$ concludes with (5.16) the proof of Item 3 (recall that the precise definition of $\mathcal{R}^{\infty}$ is given in Definition 2.2).

Finally, Item 4 is a direct consequence of Item 3.

\subsubsection{Characterization of the HUM operator for coupled wave equations}

The method used in this section follows that of Section 5.3.1. Yet the proof is more involved. In fact, here, there is a possible interaction between waves with positive and negative frequencies. For the same reason, some of the remainders that we shall obtain along the proof will only be 1-smoothing in this case, whereas we obtained infinitely smoothing remainder terms in Section 5.3.1. Note that for a scalar wave equation, such remainder terms are also 1-smoothing only. This can be improved by taking a time dependent control function $b_{\omega}(t, x)$ vanishing at all orders at times 0 and $T$ (see [DL09, 
Theorem 4.1]). Due to the coupling of two waves in System (1.1) we shall see that the remainder terms that we shall obtain cannot be better than 1-smoothing, even if $b_{\omega}$ is chosen time dependent.

Recall that proving the observability inequality (1.5) for the adjoint system (1.6) is equivalent to proving the $L^{2}-L^{2}$ observability inequality (2.7) for solutions of System (2.6):

$$
\begin{cases}P w_{1}=0 & \text { in }(0, T) \times \Omega, \\ P w_{2}=-b(x)(1-\Delta)^{\frac{1}{2}} w_{1} & \text { in }(0, T) \times \Omega .\end{cases}
$$

The symmetric setting of this system is, once again, simpler to handle, and we shall therefore work with $L^{2}-L^{2}$ data. In the framework of Section 5.1 this corresponds to the case $s=1$ and $\sigma=1$ and the associated control system is (2.8).

Given the following initial data

$$
\left(w_{1}(0), w_{2}(0), \partial_{t} w_{1}(0), \partial_{t} w_{2}(0)\right)=\left(w_{1}^{0}, w_{2}^{0}, w_{1}^{1}, w_{2}^{1}\right) \in L^{2}\left(\Omega ; \mathbb{C}^{2}\right) \times H^{-1}\left(\Omega ; \mathbb{C}^{2}\right)
$$

for System (5.17), we shall split them into their positive and negative part following [DL09]. Concerning the state $w_{1}$, we set

$$
g_{ \pm}=\frac{1}{2}\left(\Pi_{+} w_{1}^{0} \mp i \lambda^{-1} \Pi_{+} w_{1}^{1}\right) \in L_{+}^{2}(\Omega), \quad g_{0}=\Pi_{0} w_{1}^{0} \in \mathbb{C}, \quad g_{1}=\Pi_{0} w_{1}^{1} \in \mathbb{C},
$$

so that

$$
w_{1}(0)=w_{1}^{0}=g_{+}+g_{-}+g_{0}, \quad \partial_{t} w_{1}(0)=w_{1}^{1}=i \lambda\left(g_{+}-g_{-}\right)+g_{1} .
$$

In this splitting, the expression of the solution of System (5.17) is particularly simple:

$$
w_{1}(t)=e^{i t \lambda} g_{+}+e^{-i t \lambda} g_{-}+t g_{1}+g_{0} .
$$

We proceed to the same decomposition for $w_{2}$ and set

$$
h_{ \pm}=\frac{1}{2}\left(\Pi_{+} w_{2}^{0} \mp i \lambda^{-1} \Pi_{+} w_{2}^{1}\right) \in L_{+}^{2}(\Omega), \quad h_{0}=\Pi_{0} w_{2}^{0} \in \mathbb{C}, \quad h_{1}=\Pi_{0} w_{2}^{1} \in \mathbb{C},
$$

so that

$$
w_{2}(0)=w_{2}^{0}=h_{+}+h_{-}+h_{0}, \quad \partial_{t} w_{2}(0)=w_{2}^{1}=i \lambda\left(h_{+}-h_{-}\right)+h_{1} .
$$

In this splitting, the expression of the solution of System (5.17) through the Duhamel formula is

$$
\begin{aligned}
w_{2}(t)= & e^{i t \lambda} h_{+}+e^{-i t \lambda} h_{-}+t h_{1}+h_{0}-\lambda^{-1} \int_{0}^{t} \sin ((t-\sigma) \lambda) \Pi_{+} b(1-\Delta)^{\frac{1}{2}} w_{1}(\sigma) d \sigma \\
& -\int_{0}^{t}(t-\sigma) \Pi_{0} b(1-\Delta)^{\frac{1}{2}} w_{1}(\sigma) d \sigma .
\end{aligned}
$$

We denote by $\Sigma_{+}$the linear mapping

$$
\begin{aligned}
\Sigma_{+}: \quad H^{s}\left(\Omega ; \mathbb{C}^{2}\right) \times H^{s-1}\left(\Omega ; \mathbb{C}^{2}\right) & \rightarrow H_{+}^{s}\left(\Omega ; \mathbb{C}^{4}\right) \\
\left(w_{1}^{0}, w_{2}^{0}, w_{1}^{1}, w_{2}^{1}\right) & \mapsto\left(g_{+}, h_{+}, g_{-}, h_{-}\right),
\end{aligned}
$$

corresponding to the splitting (5.18)-(5.20). Note that this mapping is onto but not injective since constants are lost. Note also that the order in $\left(g_{+}, h_{+}, g_{-}, h_{-}\right)$is important since we collect together data corresponding to the same wave frequencies. We also denote by $\Sigma_{0}$ the linear mapping associated with constant functions

$$
\begin{aligned}
\Sigma_{0}: \quad H^{s}\left(\Omega ; \mathbb{C}^{2}\right) \times H^{s-1}\left(\Omega ; \mathbb{C}^{2}\right) & \rightarrow \mathbb{C}^{4} \\
\left(w_{1}^{0}, w_{2}^{0}, w_{1}^{1}, w_{2}^{1}\right) & \mapsto\left(g_{0}, h_{0}, g_{1}, h_{1}\right),
\end{aligned}
$$

corresponding to the splitting (5.18)-(5.20). Finally, we denote by $\Sigma$ the isomorphism corresponding to the splitting (5.18)-(5.20):

$$
\begin{aligned}
\Sigma: \quad H^{s}\left(\Omega ; \mathbb{C}^{2}\right) \times H^{s-1}\left(\Omega ; \mathbb{C}^{2}\right) & \rightarrow H_{+}^{s}\left(\Omega ; \mathbb{C}^{4}\right) \times \mathbb{C}^{4} \\
\mathcal{W}^{0}=\left(w_{1}^{0}, w_{2}^{0}, w_{1}^{1}, w_{2}^{1}\right) & \mapsto\left(\Sigma_{+} \mathcal{W}^{0}, \Sigma_{0} \mathcal{W}^{0}\right)=\left(g_{+}, h_{+}, g_{-}, h_{-}, g_{0}, h_{0}, g_{1}, h_{1}\right) .
\end{aligned}
$$


We recall the natural duality bracket

$$
\begin{aligned}
\left\langle\left(u_{1}^{0}, u_{2}^{0}, u_{1}^{1}, u_{2}^{1}\right),\left(w_{1}^{0}, w_{2}^{0}, w_{1}^{1}, w_{2}^{1}\right)\right\rangle_{*}= & \left(u_{1}^{1}, w_{1}^{0}\right)_{L^{2}(\Omega)}-\left\langle u_{1}^{0}, w_{1}^{1}\right\rangle_{H^{1}(\Omega), H^{-1}(\Omega)} \\
& +\left(u_{2}^{1}, w_{2}^{0}\right)_{L^{2}(\Omega)}-\left\langle u_{2}^{0}, w_{2}^{1}\right\rangle_{H^{1}(\Omega), H^{-1}(\Omega)},
\end{aligned}
$$

as used in Section 5.1 describing the Hilbert Uniqueness Method. In the case $s=0$, the transpose operator of $\Sigma$ with respect to this duality bracket

$$
\Sigma^{*}: L_{+}^{2}\left(\Omega ; \mathbb{C}^{4}\right) \times \mathbb{C}^{4} \rightarrow H^{1}\left(\Omega ; \mathbb{C}^{2}\right) \times L^{2}\left(\Omega ; \mathbb{C}^{2}\right),
$$

is given by

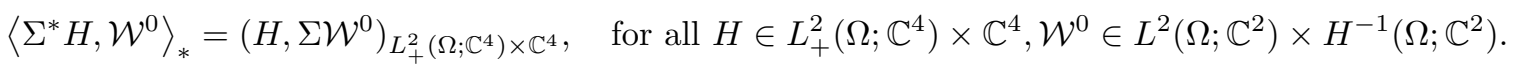

We can now state the analogue of Theorem 5.3 for full wave systems, providing a characterization of the Gramian operator (in the wave splitting (5.18)-(5.20)).

Theorem 5.5. Denoting by $\mathcal{W}^{0}=\left(w_{1}^{0}, w_{2}^{0}, w_{1}^{1}, w_{2}^{1}\right)$ the initial data for System (5.17), we have

$$
\int_{0}^{T}\left\|b_{\omega} w_{2}\right\|_{L^{2}(\Omega)}^{2} d t=\left(\mathcal{G}_{T} \Sigma \mathcal{W}^{0}, \Sigma \mathcal{W}^{0}\right)_{\left.L_{+}^{2}\left(\Omega ; \mathbb{C}^{4}\right) \times \mathbb{C}^{4}\right)},
$$

where $\mathcal{L}_{T}=\Sigma^{*} \mathcal{G}_{T} \Sigma \in \mathcal{L}\left(L^{2}\left(\Omega ; \mathbb{C}^{2}\right) \times H^{-1}\left(\Omega ; \mathbb{C}^{2}\right) ; H^{1}\left(\Omega ; \mathbb{C}^{2}\right) \times L^{2}\left(\Omega ; \mathbb{C}^{2}\right)\right)$ is the Gramian operator of (5.17), and $\mathcal{G}_{T} \in \mathcal{L}\left(L_{+}^{2}\left(\Omega ; \mathbb{C}^{4}\right) \times \mathbb{C}^{4}\right)$ is its representation in the splitting (5.18)-(5.20).

Moreover, there exist $G_{T} \in \Psi_{\mathrm{phg}}^{0}\left(\Omega ; \mathbb{C}^{4 \times 4}\right) \cap \bigcap_{s \in \mathbb{R}} \mathcal{L}\left(H_{+}^{s}\left(\Omega ; \mathbb{C}^{4}\right)\right), R_{T} \in \bigcap_{s \in \mathbb{R}} \mathcal{L}\left(H_{+}^{s}\left(\Omega ; \mathbb{C}^{4}\right)\right)$ a 1 smoothing operator, $R_{T}^{0} \in \mathcal{L}\left(\mathbb{C}^{4}\right)$, and $\tilde{R}_{T} \in \bigcap_{s \in \mathbb{R}} \mathcal{L}\left(\mathbb{C}^{4} ; H_{+}^{s}\left(\Omega ; \mathbb{C}^{4}\right)\right)$ such that $\mathcal{G}_{T}$ on $L_{+}^{2}\left(\Omega ; \mathbb{C}^{4}\right) \times \mathbb{C}^{4}$ is given by (a $8 \times 8$ operator matrix)

$$
\mathcal{G}_{T}=\left(\begin{array}{cc}
G_{T}+R_{T} & \tilde{R}_{T} \\
\tilde{R}_{T}^{*} & R_{T}^{0}
\end{array}\right) .
$$

The principal symbol of $G_{T}$ is (a $4 \times 4$ symbol matrix)

$$
\sigma_{0}\left(G_{T}\right)=\left(\begin{array}{cc}
\sigma_{0}\left(G_{T}^{+}\right) & 0 \\
0 & \sigma_{0}\left(G_{T}^{-}\right)
\end{array}\right)
$$

with $\sigma_{0}\left(G_{T}^{ \pm}\right) \in S_{\mathrm{phg}}^{0}\left(T^{*} \Omega, \mathbb{C}^{2 \times 2}\right)$,

$$
\sigma_{0}\left(G_{T}^{ \pm}\right)=\left(\begin{array}{cc}
\frac{1}{4} \int_{0}^{T} b_{\omega}^{2} \circ \varphi_{t}^{ \pm}\left(\int_{0}^{t} b \circ \varphi_{\sigma}^{ \pm} d \sigma\right)^{2} d t & \pm \frac{1}{2 i} \int_{0}^{T} b_{\omega}^{2} \circ \varphi_{t}^{ \pm}\left(\int_{0}^{t} b \circ \varphi_{\sigma}^{ \pm} d \sigma\right) d t \\
\mp \frac{1}{2 i} \int_{0}^{T} b_{\omega}^{2} \circ \varphi_{t}^{ \pm}\left(\int_{0}^{t} b \circ \varphi_{\sigma}^{ \pm} d \sigma\right) d t & \int_{0}^{T} b_{\omega}^{2} \circ \varphi_{t}^{ \pm} d t
\end{array}\right) .
$$

In particular, we have

$$
\operatorname{det}\left(\sigma_{0}\left(G_{T}\right)\right)=\operatorname{det}\left(\sigma_{0}\left(G_{T}^{+}\right)\right) \operatorname{det}\left(\sigma_{0}\left(G_{T}^{-}\right)\right)
$$

with

$$
\operatorname{det}\left(\sigma_{0}\left(G_{T}^{ \pm}\right)\right)=\frac{1}{8} \int_{0}^{T} \int_{0}^{T}\left(b_{\omega}^{2} \circ \varphi_{t_{1}}^{ \pm}\right)\left(b_{\omega}^{2} \circ \varphi_{t_{2}}^{ \pm}\right)\left(\int_{t_{1}}^{t_{2}} b \circ \varphi_{\sigma}^{ \pm} d \sigma\right)^{2} d t_{1} d t_{2} \in S_{\mathrm{phg}}^{0}\left(T^{*} \Omega\right) .
$$

From (5.22), if the operator $\mathcal{L}_{T}=\Sigma^{*} \mathcal{G}_{T} \Sigma$ is invertible then the HUM operator $\mathcal{H}_{T}$ is precisely its inverse (see Section 5.2). The following corollary provides the microlocal structure of $\mathcal{H}_{T}$ within the splitting framework. In particular, this provides a second proof of the observability of System (5.17) under the appropriate geometric conditions.

Corollary 5.6. Assume that $\omega$ and $\mathcal{O}$ satisfy $G C C$ and that $T>T_{\omega \rightarrow \mathcal{O} \rightarrow \omega}$. Then, we have the following properties: 
1. The operator $G_{T} \in \Psi_{\mathrm{phg}}^{0}\left(\Omega, \mathbb{C}^{4 \times 4}\right)$ is elliptic.

2. The operator $\mathcal{G}_{T}$ is coercive on $L_{+}^{2}\left(\Omega ; \mathbb{C}^{4}\right) \times \mathbb{C}^{4}$ :

$$
\begin{aligned}
\int_{0}^{T}\left\|b_{\omega} w_{2}\right\|_{L^{2}(\Omega)}^{2} d t & =\left(\mathcal{G}_{T} \Sigma \mathcal{W}^{0}, \Sigma \mathcal{W}^{0}\right)_{L_{+}^{2}\left(\Omega ; \mathbb{C}^{4}\right) \times \mathbb{C}^{4}} \\
& \geq C\left\|\Sigma \mathcal{W}^{0}\right\|_{L_{+}^{2}\left(\Omega ; \mathbb{C}^{4}\right) \times \mathbb{C}^{4}}^{2} \\
& \geq C^{\prime}\left\|\mathcal{W}^{0}\right\|_{L^{2}\left(\Omega ; \mathbb{C}^{2}\right) \times H^{-1}\left(\Omega ; \mathbb{C}^{2}\right)}^{2}
\end{aligned}
$$

for all $\mathcal{W}^{0} \in L^{2}\left(\Omega ; \mathbb{C}^{2}\right) \times H^{-1}\left(\Omega ; \mathbb{C}^{2}\right)$ and $\left(w_{1}, w_{2}\right)$ associated solutions of System (5.17).

3. The operator $\mathcal{G}_{T}$ is invertible on $\mathcal{L}\left(L_{+}^{2}\left(\Omega ; \mathbb{C}^{4}\right) \times \mathbb{C}^{4}\right)$. The HUM operator (in the splitting (5.18)$(5.20)$ ) is its inverse $\mathcal{G}_{T}^{-1}$ and can be decomposed on $L_{+}^{2}\left(\Omega ; \mathbb{C}^{4}\right) \times \mathbb{C}^{4}$ as (the $8 \times 8$ operator matrix)

$$
\mathcal{G}_{T}^{-1}=\left(\begin{array}{cc}
\Lambda_{T}+S_{T} & \tilde{S}_{T} \\
\tilde{S}_{T}^{*} & S_{T}^{0}
\end{array}\right)
$$

where $S_{T} \in \mathcal{R}^{1}, S_{T}^{0} \in \mathcal{L}\left(\mathbb{C}^{4}\right), \tilde{S}_{T} \in \bigcap_{s \in \mathbb{R}} \mathcal{L}\left(\mathbb{C}^{4} ; H_{+}^{s}\left(\Omega ; \mathbb{C}^{4}\right)\right)$ and $\Lambda_{T} \in S_{\mathrm{phg}}^{0}\left(T^{*} \Omega, \mathbb{C}^{4 \times 4}\right) \cap$ $\bigcap_{s \in \mathbb{R}} \mathcal{L}\left(H_{+}^{s}\left(\Omega ; \mathbb{C}^{4}\right)\right)$, with principal symbol

$$
\sigma_{0}\left(\Lambda_{T}\right)=\sigma_{0}\left(G_{T}\right)^{-1}=\left(\begin{array}{cc}
\sigma_{0}\left(G_{T}^{+}\right)^{-1} & 0 \\
0 & \sigma_{0}\left(G_{T}^{-}\right)^{-1}
\end{array}\right)
$$

4. In particular, the $H U M$ operator $\mathcal{G}_{T}^{-1}$ is an isomorphism of $H_{+}^{s}\left(\Omega ; \mathbb{C}^{4}\right) \times \mathbb{C}^{4}$ for all $s \geq 0$ and we have

$$
\mathrm{WF}^{s}\left(\left(\mathcal{G}_{T}\right)^{-1} \Sigma \mathcal{W}^{0}\right)=\mathrm{WF}^{s}\left(\Sigma \mathcal{W}^{0}\right) .
$$

Recall that the definition of the $H^{s}$-wavefront set of a $k$-tuple is given in (5.11).

Note that in Theorem 5.5, we do not use any sign assumption on the coupling term $b$, but only that $b$ is real valued. As a consequence, we can state in this more general case a criterium for the high-frequency controllability of System (1.1). Compare with Theorem 1.3.

Definition 5.7. We say that $(\omega, b, T)$ satisfies the Polarization Control Condition (PCC) if, for any $\rho \in S^{*} \Omega$, there exists $0<t_{1}^{ \pm}<t_{2}^{ \pm}<T$ such that

$$
\varphi_{t_{1}^{ \pm}}^{ \pm}(\rho) \in \omega, \quad \varphi_{t_{2}^{ \pm}}^{ \pm}(\rho) \in \omega, \quad \text { and } \quad \int_{t_{1}}^{t_{2}} b \circ \varphi_{\sigma}^{ \pm}(\rho) d \sigma \neq 0
$$

In particular, this requires that both $\omega$ and the set $\{b \neq 0\}$ satisfy GCC. Note that if $b \geq 0$, we have that $(\omega, b, T)$ satisfies PCC if and only if $\omega$ and $\mathcal{O}:=\{b>0\}$ both satisfy GCC and $T>T_{\omega \rightarrow \mathcal{O} \rightarrow \omega}$. With this definition, we have the following result.

Corollary 5.8. $\quad$ 1. The weak observability inequality

$$
\int_{0}^{T}\left\|b_{\omega} w_{2}\right\|_{L^{2}(\Omega)}^{2} d t \geq C\left\|\mathcal{W}^{0}\right\|_{L^{2}\left(\Omega ; \mathbb{C}^{2}\right) \times H^{-1}\left(\Omega ; \mathbb{C}^{2}\right)}^{2}-\left\|\mathcal{W}^{0}\right\|_{H^{-1}\left(\Omega ; \mathbb{C}^{2}\right) \times H^{-2}\left(\Omega ; \mathbb{C}^{2}\right)}^{2},
$$

holds for all $\mathcal{W}^{0} \in L^{2}\left(\Omega ; \mathbb{C}^{2}\right) \times H^{-1}\left(\Omega ; \mathbb{C}^{2}\right)$ and $\left(w_{1}, w_{2}\right)$ associated solutions of System (5.17), if and only if $(\omega, b, T)$ satisfies $P C C$.

2. Suppose that $(\omega, b, T)$ satisfies PCC and that no eigenfunction $\left(\varphi_{1}, \varphi_{2}\right)$ of the operator

$$
\left(\begin{array}{cc}
-\Delta & 0 \\
b(1-\Delta)^{\frac{1}{2}} & -\Delta
\end{array}\right)
$$


satisfy $\left.\varphi_{2}\right|_{\omega}=0$. Then, we have the observability inequality

$$
\int_{0}^{T}\left\|b_{\omega} w_{2}\right\|_{L^{2}(\Omega)}^{2} d t \geq C\left\|\mathcal{W}^{0}\right\|_{L^{2}\left(\Omega ; \mathbb{C}^{2}\right) \times H^{-1}\left(\Omega ; \mathbb{C}^{2}\right)}^{2},
$$

for all $\mathcal{W}^{0} \in L^{2}\left(\Omega ; \mathbb{C}^{2}\right) \times H^{-1}\left(\Omega ; \mathbb{C}^{2}\right)$ and $\left(w_{1}, w_{2}\right)$ associated solutions of System (5.17) (i.e. System (1.1) is controllable).

Note that the additional unique continuation assumption is valid in the following two particular cases

- $b \geq 0$ on $\Omega$ and $\{b>0\} \neq \emptyset$ (see Section 3.2 and Remark 3.5);

- $\omega \cap\{b \neq 0\} \neq \emptyset$ (see [Léa10, Proposition 5.1]).

The question seems to be open in the general case.

Proof of Theorem 5.5. Here, we mostly follow the proof of Theorem 5.3. In fact, several additional terms appear in the calculations that we have to deal with.

In the matrix $\mathcal{G}_{T}$, given in (5.23), each one of the four blocks is a $4 \times 4$ matrix of operators (or simply scalars numbers). In a first step, we check that all blocks have the announced form, excluding the first block. In a second step, we shall focus on this first block, that contains all the high-frequency of $\mathcal{G}_{T}$. We wish to compute $\int_{0}^{T}\left\|b_{\omega} w_{2}\right\|_{L^{2}(\Omega)}^{2} d t$ and to cast it in the form of the right hand-side of $(5.22)$, that is $\left(\mathcal{G}_{T} H, H\right)_{\left.L_{+}^{2}\left(\Omega ; \mathbb{C}^{4}\right) \times \mathbb{C}^{4}\right)}$, with $H=\Sigma \mathcal{W}^{0}$.

Focusing on (5.19)-(5.21), we first remark that $\left(\Pi_{0} w_{2}(t), e_{0}\right)_{L^{2}(\Omega)} \in \mathscr{C}^{\infty}(\mathbb{R} ; \mathbb{C})$. Hence, for all $t \in \mathbb{R}$, the map

$$
H=\Sigma \mathcal{W}^{0} \mapsto\left(\Pi_{0} w_{2}(t), e_{0}\right)_{L^{2}(\Omega)}
$$

is an infinitely smoothing operator. As a consequence, it suffices to carry out our computation with $w_{2}$ replaced by

$$
\Pi_{+} w_{2}(t)=e^{i t \lambda} h_{+}+e^{-i t \lambda} h_{-}-\lambda^{-1} \int_{0}^{t} \sin ((t-\sigma) \lambda) \Pi_{+} b(1-\Delta)^{\frac{1}{2}}\left(e^{i \sigma \lambda} g_{+}+e^{-i \sigma \lambda} g_{-}+\sigma g_{1}+g_{0}\right) d \sigma,
$$

that is, ignoring the components along $e_{0}$, which contribute to the smoothing operators in the expression of $\mathcal{G}_{T}$ in $(5.23)$. In the previous expression, the term

$$
\lambda^{-1} \int_{0}^{t} \sin ((t-\sigma) \lambda) \Pi_{+} b(1-\Delta)^{\frac{1}{2}}\left(\sigma g_{1}+g_{0}\right) d \sigma
$$

is in $\mathscr{C}^{\infty}(\mathbb{R} \times \Omega)$ since the functions $g_{1}=g_{1} e_{0}$ and $g_{0}=g_{0} e_{0}$ are smooth. As a consequence, we have

$$
\begin{aligned}
\int_{0}^{T}\left\|b_{\omega} w_{2}\right\|_{L^{2}(\Omega)}^{2} d t= & \int_{0}^{T}\left(R_{t} H, H\right)_{L^{2}(\Omega)} d t+\int_{0}^{T} \| b_{\omega} e^{i t \lambda} h_{+}+b_{\omega} e^{-i t \lambda} h_{-} \\
& \quad-b_{\omega} \lambda^{-1} \int_{0}^{t} \sin ((t-\sigma) \lambda) \Pi_{+} b(1-\Delta)^{\frac{1}{2}}\left(e^{i \sigma \lambda} g_{+}+e^{-i \sigma \lambda} g_{-}\right) d \sigma \|_{L^{2}(\Omega)}^{2} d t
\end{aligned}
$$

Now, we focus on the first block of the operator matrix in (5.23), i.e. a $4 \times 4$ matrix of operators on $\Omega$, that yields the bilinear form applied to $\left(g_{+}, h_{+}, g_{-}, h_{-}\right)=\Sigma_{+}\left(w_{1}^{0}, w_{2}^{0}, w_{1}^{1}, w_{2}^{1}\right)$. It is associated with the last term in the expression (5.27), given by

$\int_{0}^{T}\left\|b_{\omega}\left(e^{i t \lambda} h_{+}+e^{-i t \lambda} h_{-}-\frac{\lambda^{-1}}{2 i} \int_{0}^{t}\left(e^{i(t-\sigma) \lambda}-e^{-i(t-\sigma) \lambda}\right) \Pi_{+} b(1-\Delta)^{\frac{1}{2}}\left(e^{i \sigma \lambda} g_{+}+e^{-i \sigma \lambda} g_{-}\right) d \sigma\right)\right\|_{L^{2}(\Omega)}^{2} d t$

We only compute the first two lines of this matrix (as operating on $\left(g_{+}, h_{+}, g_{-}, h_{-}\right)$) and denote by $\mathcal{G}_{T}(\cdot, \cdot)$ the associated term in this matrix. The computation of the last two lines is similar and is left to the reader. 
Let us start with the (simpler) second line. We have

$$
\mathcal{G}_{T}\left(h_{+}, h_{+}\right)=\int_{0}^{T}\left(b_{\omega} e^{i t \lambda} h_{+}, b_{\omega} e^{i t \lambda} h_{+}\right)_{L^{2}(\Omega)} d t=\left(\int_{0}^{T} e^{-i t \lambda} b_{\omega}^{2} e^{i t \lambda} d t h_{+}, h_{+}\right)_{L^{2}(\Omega)} .
$$

Next, we compute

$$
\begin{aligned}
\mathcal{G}_{T}\left(h_{+}, h_{-}\right) & =\int_{0}^{T}\left(b_{\omega} e^{i t \lambda} h_{+}, b_{\omega} e^{-i t \lambda} h_{-}\right)_{L^{2}(\Omega)} d t=\left(\int_{0}^{T} e^{i t \lambda} b_{\omega}^{2} e^{i t \lambda} d t h_{+}, h_{-}\right)_{L^{2}(\Omega)} \\
& =\left(R_{T} h_{+}, h_{-}\right)_{L^{2}(\Omega)},
\end{aligned}
$$

for some 1-smoothing ${ }^{2}$ operator $R_{T}$, according to Lemma A.1 (see Appendix A). Similarly, we compute

$$
\begin{aligned}
-\mathcal{G}_{T}\left(h_{+}, g_{+}\right) & =\int_{0}^{T}\left(b_{\omega} e^{i t \lambda} h_{+}, b_{\omega} \frac{\lambda^{-1}}{2 i} \int_{0}^{t}\left(e^{i(t-\sigma) \lambda}-e^{-i(t-\sigma) \lambda}\right) \Pi_{+} b(1-\Delta)^{\frac{1}{2}} e^{i \sigma \lambda} d \sigma g_{+}\right)_{L^{2}(\Omega)} d t \\
& =\int_{0}^{T}\left(b_{\omega} e^{i t \lambda} h_{+}, b_{\omega} \frac{1}{2 i} \int_{0}^{t}\left(e^{i(t-\sigma) \lambda}-e^{-i(t-\sigma) \lambda}\right) \Pi_{+} b e^{i \sigma \lambda} d \sigma g_{+}+R_{t} g_{+}\right)_{L^{2}(\Omega)} d t,
\end{aligned}
$$

for some continuous family of 1 -smoothing operators $R_{t}$, since $(1-\Delta)^{\frac{1}{2}}-\lambda \in \Psi_{\mathrm{phg}}^{0}(\Omega)$ and $[b, \lambda] \in$ $\Psi_{\text {phg }}^{0}(\Omega)$. We obtain

$$
\begin{aligned}
\mathcal{G}_{T}\left(h_{+}, g_{+}\right)= & \int_{0}^{T}\left(\frac{1}{2 i} \Pi_{+}\left(\int_{0}^{t} e^{-i \sigma \lambda} b e^{i \sigma \lambda} d \sigma\right) \Pi_{+} e^{-i t \lambda} b_{\omega}^{2} e^{i t \lambda} h_{+}, g_{+}\right)_{L^{2}(\Omega)} d t \\
& -\int_{0}^{T}\left(\frac{1}{2 i} \Pi_{+}\left(\int_{0}^{t} e^{-i \sigma \lambda} b e^{-i \sigma \lambda} d \sigma\right) \Pi_{+} e^{i t \lambda} b_{\omega}^{2} e^{i t \lambda} h_{+}, g_{+}\right)_{L^{2}(\Omega)} d t+\left(R_{T} h_{+}, g_{+}\right)_{L^{2}(\Omega)},
\end{aligned}
$$

where $R_{T}$ is again a continuous family of 1-smoothing operators. In the central term of the right handside, we notice that $\int_{0}^{t} e^{-i \sigma \lambda} b e^{-i \sigma \lambda} d \sigma$ is a continuous family of 1 -smoothing operators ${ }^{3}$ according to Lemma A.1. With the Egorov Theorem (Theorem 2.1), we define as in (5.13) the pseudodifferential operators

$$
B_{t}^{ \pm}:=\int_{0}^{t} e^{\mp i \sigma \lambda} b e^{ \pm i \sigma \lambda} d \sigma \in \Psi_{\mathrm{phg}}^{0}(\Omega)
$$

with principal symbol

$$
\sigma_{0}\left(B_{t}^{ \pm}\right)(x, \eta)=\int_{0}^{t} b \circ \varphi_{\sigma}^{ \pm}(x, \eta) d \sigma, \quad(x, \eta) \in T^{*} \Omega,
$$

we now have

$$
\mathcal{G}_{T}\left(h_{+}, g_{+}\right)=\left(\frac{1}{2 i} \int_{0}^{T} \Pi_{+} B_{t}^{+} \Pi_{+} e^{-i t \lambda} b_{\omega}^{2} e^{i t \lambda} d t h_{+}, g_{+}\right)_{L^{2}(\Omega)}+\left(R_{T} h_{+}, g_{+}\right)_{L^{2}(\Omega)},
$$

where $R_{T}$ is a continuous family of 1 -smoothing operators. Similarly, we compute

$$
\begin{aligned}
\mathcal{G}_{T}\left(h_{+}, g_{-}\right)= & -\int_{0}^{T}\left(b_{\omega} e^{i t \lambda} h_{+}, b_{\omega} \frac{1}{2 i} \int_{0}^{t}\left(e^{i(t-\sigma) \lambda}-e^{-i(t-\sigma) \lambda}\right) \Pi_{+} b e^{-i \sigma \lambda} g_{-}+R_{t} g_{-}\right)_{L^{2}(\Omega)} d t \\
= & \left(\frac{1}{2 i} \int_{0}^{T} \Pi_{+}\left(\int_{0}^{t} e^{i \sigma \lambda} b e^{i \sigma \lambda} d \sigma\right) \Pi_{+} e^{-i t \lambda} b_{\omega}^{2} e^{i t \lambda} d t h_{+}, g_{-}\right)_{L^{2}(\Omega)} \\
& -\left(\frac{1}{2 i} \int_{0}^{T} \Pi_{+}\left(\int_{0}^{t} e^{+i \sigma \lambda} b e^{-i \sigma \lambda} d \sigma\right) \Pi_{+} e^{i t \lambda} b_{\omega}^{2} e^{i t \lambda} d t h_{+}, g_{-}\right)_{L^{2}(\Omega)}+\left(R_{T} h_{+}, g_{-}\right)_{L^{2}(\Omega)} .
\end{aligned}
$$

\footnotetext{
${ }^{2}$ This smoothing term can be maid infinitely smoothing if $b_{\omega}$ is chosen vanishing at infinite order at $t=0$ and $t=T$ (see [DL09]).

${ }^{3}$ It seems to us that no particular choice of $b$ (except $b=0$ ) can improve this smoothing property.
} 
According to Lemma A.1, the operator $\int_{0}^{t} e^{i \sigma \lambda} b e^{i \sigma \lambda} d \sigma$ is a continuous family of 1-smoothing operators, and so is the first term in the right hand-side of this expression. The second term is also a continuous family of 1-smoothing operators, using Lemma A.2. We thus obtain

$$
\mathcal{G}_{T}\left(h_{+}, g_{-}\right)=\left(R_{T} h_{+}, g_{-}\right)_{L^{2}(\Omega)},
$$

where $R_{T}$ is 1 -smoothing.

We have already computed the second line of the first block of the matrix $\mathcal{G}_{T}$. Let us now compute the first line. First, we have

$$
\mathcal{G}_{T}\left(g_{+}, g_{+}\right)=\int_{0}^{T}\left\|b_{\omega} \frac{1}{2 i} \int_{0}^{t}\left(e^{i(t-\sigma) \lambda}-e^{-i(t-\sigma) \lambda}\right) \Pi_{+} b e^{i \sigma \lambda} g_{+}+R_{t} g_{+}\right\|_{L^{2}(\Omega)}^{2} d t
$$

and we notice that, according to Lemma A.1, $b_{\omega} \frac{1}{2 i} \int_{0}^{t} e^{-i(t-\sigma) \lambda} \Pi_{+} b e^{i \sigma \lambda} d \sigma$ is a continuous family of 1-smoothing operators. Hence, we have (with a continuous family of 1-smoothing operators $\tilde{R}_{t}$ )

$$
\begin{aligned}
\mathcal{G}_{T}\left(g_{+}, g_{+}\right) & =\int_{0}^{T}\left\|b_{\omega} \frac{1}{2 i} \int_{0}^{t} e^{i(t-\sigma) \lambda} \Pi_{+} b e^{i \sigma \lambda} g_{+}+\tilde{R}_{t} g_{+}\right\|_{L^{2}(\Omega)}^{2} d t \\
& =\frac{1}{4}\left(\Pi_{+} \int_{0}^{T}\left(B_{t}^{+}\right)^{*} \Pi_{+} e^{-i t \lambda} b_{\omega}^{2} e^{i t \lambda} \Pi_{+} B_{t}^{+} d t \Pi_{+} g_{+}, g_{+}\right)_{L^{2}(\Omega)}+\left(R_{T} g_{+}, g_{+}\right)_{L^{2}(\Omega)},
\end{aligned}
$$

as in the proof of Theorem 5.3.

Then, the operator arising in the term $\mathcal{G}_{T}\left(g_{+}, h_{+}\right)$is the adjoint of that of $\mathcal{G}_{T}\left(h_{+}, g_{+}\right)$, given by (5.31).

Next

$$
\begin{aligned}
\mathcal{G}_{T}\left(g_{+}, h_{-}\right) & =\int_{0}^{T}\left(e^{i t \lambda} b_{\omega}^{2} \frac{\lambda^{-1}}{2 i} \int_{0}^{t}\left(e^{i(t-\sigma) \lambda}-e^{-i(t-\sigma) \lambda}\right) \Pi_{+} b(1-\Delta)^{\frac{1}{2}} e^{i \sigma \lambda} d \sigma g_{+}, h_{-}\right)_{L^{2}(\Omega)} d t \\
& =\frac{1}{2 i} \int_{0}^{T}\left(e^{i t \lambda} b_{\omega}^{2} e^{i t \lambda} \int_{0}^{t} e^{-i \sigma \lambda} \Pi_{+} b e^{i \sigma \lambda} d \sigma g_{+}+R_{t} g_{+}, h_{-}\right)_{L^{2}(\Omega)} d t
\end{aligned}
$$

since $\int_{0}^{t} e^{-i(t-\sigma) \lambda} \Pi_{+} b e^{i \sigma \lambda} d \sigma$ is a continuous family of 1-smoothing operators according to Lemma A.1. Lemma A.2 then yields

$$
\mathcal{G}_{T}\left(g_{+}, h_{-}\right)=\left(R_{T} g_{+}, h_{-}\right)_{L^{2}(\Omega)}
$$

for $R_{T}$ some 1-smoothing operator.

It only remains to compute

$$
\begin{aligned}
& \mathcal{G}_{T}\left(g_{+}, g_{-}\right)=- \int_{0}^{T}\left(b_{\omega} \frac{\lambda^{-1}}{2 i} \int_{0}^{t}\left(e^{i(t-\sigma) \lambda}-e^{-i(t-\sigma) \lambda}\right) \Pi_{+} b(1-\Delta)^{\frac{1}{2}} e^{i \sigma \lambda} d \sigma g_{+},\right. \\
&\left.b_{\omega} \frac{\lambda^{-1}}{2 i} \int_{0}^{t}\left(e^{i(t-\sigma) \lambda}-e^{-i(t-\sigma) \lambda}\right) \Pi_{+} b(1-\Delta)^{\frac{1}{2}} e^{-i \sigma \lambda} d \sigma g_{-}\right)_{L^{2}(\Omega)} d t \\
&=\int_{0}^{T}\left(b_{\omega} \frac{1}{2 i} \int_{0}^{t} e^{i(t-\sigma) \lambda} \Pi_{+} b e^{i \sigma \lambda} d \sigma g_{+}, b_{\omega} \frac{1}{2 i} \int_{0}^{t} e^{-i(t-\sigma) \lambda} \Pi_{+} b e^{-i \sigma \lambda} d \sigma g_{-}\right)_{L^{2}(\Omega)} d t \\
& \quad+\left(R_{T} g_{+}, g_{-}\right)_{L^{2}(\Omega)},
\end{aligned}
$$

after having used twice Lemma A.1. This can be rewritten as

$$
\begin{aligned}
\mathcal{G}_{T}\left(g_{+}, g_{-}\right) & =\int_{0}^{T}\left(b_{\omega} \frac{1}{2 i} e^{i t \lambda} B_{t}^{+} g_{+}, b_{\omega} \frac{1}{2 i} e^{-i t \lambda} B_{t}^{-} g_{-}\right)_{L^{2}(\Omega)} d t \\
& =\frac{1}{4}\left(\int_{0}^{T}\left(B_{t}^{-}\right)^{*} e^{i t \lambda} b_{\omega}^{2} \frac{1}{2 i} e^{i t \lambda} B_{t}^{+} d t g_{+}, g_{-}\right)_{L^{2}(\Omega)} .
\end{aligned}
$$


Using Lemma A.2, we finally obtain

$$
\mathcal{G}_{T}\left(g_{+}, g_{-}\right)=\left(R_{T} g_{+}, g_{-}\right)_{L^{2}(\Omega)},
$$

for $R_{T}$ some 1-smoothing operator.

Finally, combining (5.28),(5.29), (5.31), (5.32), (5.33), (5.34) and (5.35), we obtain the first two lines of the first block in (5.23) (i.e. the term $G_{T}+R_{T}$ ), with symbols according to (5.24)-(5.25). The last two lines can be computed similarly. The determinant of $\sigma_{0}\left(G_{T}\right)$ is then given by Theorem 5.3. This concludes the proof of Theorem 5.5.

Proof of Corollary 5.6. The proof of Corollary 5.6 is very similar to that of Corollary 5.4, although more technical. The proof of Item 1 is the same as that of Item 1 in Corollary 5.4.

Then, the observability inequality of Item 2 is again proved in two steps. The ellipticity of $G_{T}$ together with the Gårding inequality first yield the weak observability estimate

$$
\int_{0}^{T}\left\|b_{\omega} w_{2}\right\|_{L^{2}(\Omega)}^{2} d t=\left(\mathcal{G}_{T} \Sigma \mathcal{W}^{0}, \Sigma \mathcal{W}^{0}\right)_{L_{+}^{2}\left(\Omega ; \mathbb{C}^{4}\right) \times \mathbb{C}^{4}} \geq C\left\|\Sigma \mathcal{W}^{0}\right\|_{L_{+}^{2}\left(\Omega ; \mathbb{C}^{4}\right) \times \mathbb{C}^{4}}^{2}-C^{\prime}\left\|\Sigma \mathcal{W}^{0}\right\|_{H_{+}^{-1}\left(\Omega ; \mathbb{C}^{4}\right) \times \mathbb{C}^{4}}^{2},
$$

for all $\mathcal{W}^{0} \in L^{2}\left(\Omega ; \mathbb{C}^{2}\right) \times H^{-1}\left(\Omega ; \mathbb{C}^{2}\right)$ and $\left(w_{1}, w_{2}\right)$ associated solutions of System (5.17). Then, the proof of the observability inequality (5.26) assuming the weak observability inequality (5.36) is already done in Section 3.2.

Next, to prove Items 3 and 4 , we first remark that $\mathcal{G}_{T}$ is invertible on $\mathcal{L}\left(L_{+}^{2}\left(\Omega ; \mathbb{C}^{4}\right) \times \mathbb{C}^{4}\right)$, as for the proof of Item 3 in Corollary 5.4. This yields in particular that the operator $L_{T}:=G_{T}+R_{T} \in$ $\mathcal{L}\left(H_{+}^{s}\left(\Omega ; \mathbb{C}^{4}\right)\right.$ ) (for any $\left.s \in \mathbb{R}\right)$ is invertible in $\mathcal{L}\left(L_{+}^{2}\left(\Omega ; \mathbb{C}^{4}\right)\right.$ ). Let us show (by induction) that its inverse $L_{T}^{-1}$ in $\mathcal{L}\left(L_{+}^{2}\left(\Omega ; \mathbb{C}^{4}\right)\right.$ is in $\mathcal{L}\left(H_{+}^{s}\left(\Omega ; \mathbb{C}^{4}\right)\right)$ for any $s \geq 0$. This is true for $s=0$. Now, suppose $L_{T}^{-1} \in \mathcal{L}\left(H_{+}^{s-1}\left(\Omega ; \mathbb{C}^{4}\right)\right)$ and take $f \in H_{+}^{s}\left(\Omega ; \mathbb{C}^{4}\right)$. The equation $L_{T} u=f$ has hence a solution $u \in H_{+}^{s-1}\left(\Omega ; \mathbb{C}^{4}\right)$, sastisfying

$$
G_{T} u=f-R_{T} u \in H_{+}^{s}\left(\Omega ; \mathbb{C}^{4}\right),
$$

since $R_{T}$ is a 1-smoothing operator preserving any $H_{+}^{s}$. Using the ellipticity of the operator $G_{T} \in$ $\Psi_{\text {phg }}^{0}\left(\Omega, \mathbb{C}^{4 \times 4}\right)$, proved in Item 1 , this yields $u \in H^{s}\left(\Omega ; \mathbb{C}^{4}\right)$, and hence $L_{T}^{-1} \in \mathcal{L}\left(H_{+}^{s}\left(\Omega ; \mathbb{C}^{4}\right)\right)$. By induction, we have

$$
L_{T}^{-1} \in \mathcal{L}\left(H_{+}^{s}\left(\Omega ; \mathbb{C}^{4}\right)\right), \quad \text { for all } s \geq 0 .
$$

Now, we come back to the description of the inverse operator $\mathcal{G}_{T}^{-1}$ of $\mathcal{G}_{T}$ in $\mathcal{L}\left(L_{+}^{2}\left(\Omega ; \mathbb{C}^{4}\right) \times \mathbb{C}^{4}\right)$, which takes the form

$$
\mathcal{G}_{T}^{-1}=\left(\begin{array}{cc}
\tilde{\Lambda}_{T} & \tilde{S}_{T} \\
\tilde{S}_{T}^{*} & S_{T}^{0}
\end{array}\right)
$$

for some $\tilde{\Lambda}_{T} \in \mathcal{L}\left(L_{+}^{2}\left(\Omega ; \mathbb{C}^{4}\right)\right), S_{T}^{0} \in \mathcal{L}\left(\mathbb{C}^{4}\right)$, and $\tilde{S}_{T} \in \mathcal{L}\left(\mathbb{C}^{4} ; L_{+}^{2}\left(\Omega ; \mathbb{C}^{4}\right)\right)$. Writing now $\mathcal{G}_{T} \mathcal{G}_{T}^{-1}=\mathrm{Id}$ yields in particular

$$
L_{T} \tilde{\Lambda}_{T}+\tilde{R}_{T} \tilde{S}_{T}^{*}=\operatorname{Id}_{\mathcal{L}\left(L_{+}^{2}\left(\Omega ; \mathbb{C}^{4}\right)\right)} \quad \text { and } \quad L_{T} \tilde{S}_{T}+\tilde{R}_{T} S_{T}^{0}=0_{\mathcal{L}\left(\mathbb{C}^{4} ; L_{+}^{2}\left(\Omega ; \mathbb{C}^{4}\right)\right)}
$$

The first of these two identities together with (5.37) yields

$$
\tilde{\Lambda}_{T}=L_{T}^{-1}-L_{T}^{-1} \tilde{R}_{T} \tilde{S}_{T}^{*} \in \mathcal{L}\left(H_{+}^{s}\left(\Omega ; \mathbb{C}^{4}\right)\right), \quad \text { for all } s \geq 0 .
$$

Similarly, the second expression together with (5.37) gives

$$
\tilde{S}_{T}=-L_{T}^{-1} \tilde{R}_{T} S_{T}^{0} \in \bigcap_{s \in \mathbb{R}} \mathcal{L}\left(\mathbb{C}^{4} ; H_{+}^{s}\left(\Omega ; \mathbb{C}^{4}\right)\right) .
$$

We finally prove that $\tilde{\Lambda}_{T}$ takes the form claimed in the statement of the corollary. Since $G_{T} \in$ $\Psi_{\mathrm{phg}}^{0}\left(\Omega, \mathbb{C}^{4 \times 4}\right)$ is elliptic, there exists (see for instance [Hör85, Theorem 18.1.24]) a parametrix $\Lambda_{T} \in$ $\Psi_{\text {phg }}^{0}\left(\Omega, \mathbb{C}^{2 \times 2}\right)$ such that

$$
\Lambda_{T} G_{T}=\mathrm{Id}+R, \quad \text { with } \quad R \in \Psi_{\mathrm{phg}}^{-\infty}\left(\Omega, \mathbb{C}^{4 \times 4}\right) \quad \text { and } \quad \sigma_{0}\left(\Lambda_{T}\right)=\sigma_{0}\left(G_{T}\right)^{-1} .
$$


Applying this parametrix to the equation $\left(G_{T}+R_{T}\right) \tilde{\Lambda}_{T}+\tilde{R}_{T} \tilde{S}_{T}^{*}=$ Id yields

$$
\tilde{\Lambda}_{T}=\Lambda_{T}-\Lambda_{T} R_{T} \tilde{\Lambda}_{T}-\Lambda_{T} \tilde{R}_{T} \tilde{S}_{T}^{*}-R \tilde{\Lambda}_{T} .
$$

Then, the operator $-\Lambda_{T} \tilde{R}_{T} \tilde{S}_{T}^{*}-R \tilde{\Lambda}_{T} \in \mathcal{R}^{\infty}$ and $\Lambda_{T} R_{T} \tilde{\Lambda}_{T} \in \mathcal{R}^{1}$ according to (5.38) and as $R_{T}$ is 1-smoothing. This concludes the proof of Item 3 and the first part of Item 4.

Finally, the second part of Item 4 (the wavefront set identity) is a consequence of the pseudodifferential nature of the principal part of the operator $\mathcal{G}_{T}^{-1}$.

Proof of Corollary 5.8. The key point here is that Condition PCC is equivalent to the fact that $\rho \mapsto \operatorname{det}\left(\sigma_{0}\left(G_{T}\right)\right)(\rho)$ does not vanish for $\rho \in S^{*} \Omega$. If PCC is satisfied, then the operator $G_{T}$ is elliptic and we can follow the proof of Corollary 5.4. Similarly Item 2 only concerns the low-frequency problem and its proof follows Section 3.2.

Conversely, if PCC is not satisfied, there exists $\nu_{0} \in S^{*} \Omega$ such that $\operatorname{det}\left(\sigma_{0}\left(G_{T}\right)\left(\nu_{0}\right)\right)=0$. Hence, there exists a vector $v \in \mathbb{C}^{4} \backslash\{0\}$ such that

$$
\sigma_{0}\left(G_{T}\right)\left(\nu_{0}\right) v=0
$$

After a linear change of coordinates in $\mathbb{C}^{4}$, we may assume that $v=(1,0,0,0)$. Consider now a sequence $\left(w_{0}^{k}\right)_{k \in \mathbb{N}}$ of scalar functions on $\Omega$ such that

$$
\lim _{k \rightarrow \infty}\left\|w_{0}^{k}\right\|_{L^{2}(\Omega)}=1, \quad w_{0}^{k} \rightarrow 0 \text { in } L^{2}(\Omega ; \mathbb{C}),
$$

and $\left(w_{0}^{k}\right)_{k \in \mathbb{N}}$ is pure and admits the microlocal defect measure $\delta_{(x, \eta)=\nu_{0}}$ (such a sequence is constructed in Appendix B.2, see Equation (B.5)). The vectorial sequence $\left(u^{k}\right)_{k \in \mathbb{N}}$ given by $u^{k}:=$ $\Pi_{+} w_{0}^{k} v=\left(\Pi_{+} w_{0}^{k}, 0,0,0\right)$ satisfies (since $\Pi_{0} w_{0}^{k} \rightarrow 0$ because of the weak convergence)

$$
\lim _{k \rightarrow \infty}\left\|u^{k}\right\|_{L^{2}\left(\Omega ; \mathbb{C}^{4}\right)}=1, \quad u^{k} \rightarrow 0 \text { in } L^{2}\left(\Omega ; \mathbb{C}^{4}\right),
$$

is also pure, and admits the microlocal defect measure

$$
\mu=\left(\begin{array}{cccc}
\delta_{\nu_{0}} & 0 & 0 & 0 \\
0 & 0 & 0 & 0 \\
0 & 0 & 0 & 0 \\
0 & 0 & 0 & 0
\end{array}\right)
$$

We compute

$$
\begin{aligned}
\lim _{k \rightarrow \infty}\left(\mathcal{G}_{T}\left(\begin{array}{c}
u^{k} \\
0_{\mathbb{C}^{4}}
\end{array}\right),\left(\begin{array}{c}
u^{k} \\
0_{\mathbb{C}^{4}}
\end{array}\right)\right)_{L_{+}^{2}\left(\Omega ; \mathbb{C}^{4}\right) \times \mathbb{C}^{4}} & =\lim _{k \rightarrow \infty}\left(\left(G_{T}+R_{T}\right) u^{k}, u^{k}\right)_{L_{+}^{2}\left(\Omega ; \mathbb{C}^{4}\right)} \\
& =\lim _{k \rightarrow \infty}\left(G_{T} u^{k}, u^{k}\right)_{L^{2}\left(\Omega ; \mathbb{C}^{4}\right)},
\end{aligned}
$$

since $R_{T}$ is 1 -smoothing. Moreover, we have

$$
\left(G_{T} u^{k}, u^{k}\right)_{L^{2}\left(\Omega ; \mathbb{C}^{4}\right)} \rightarrow \int_{S^{*} \Omega} \operatorname{tr}\left\{\sigma_{0}\left(G_{T}\right)(\rho) \mu(d \rho)\right\} .
$$

Writing $\sigma_{0}\left(G_{T}\right)(\rho)=\sigma_{i j}(\rho)$, for $i, j \in\{1,2,3,4\}$, this yields

$$
\left(G_{T} u^{k}, u^{k}\right)_{L^{2}\left(\Omega ; \mathbb{C}^{4}\right)} \rightarrow\left\langle\delta_{\nu_{0}}, \sigma_{11}\right\rangle_{S^{*} \Omega}=\sigma_{11}\left(\nu_{0}\right) .
$$

Besides, condition (5.39) then gives $\sigma_{1 j}\left(\nu_{0}\right)=0$ for $j \in\{1,2,3,4\}$, so that

$$
\left(\mathcal{G}_{T}\left(\begin{array}{c}
u^{k} \\
0_{\mathbb{C}^{4}}
\end{array}\right),\left(\begin{array}{c}
u^{k} \\
0_{\mathbb{C}^{4}}
\end{array}\right)\right)_{L_{+}^{2}\left(\Omega ; \mathbb{C}^{4}\right) \times \mathbb{C}^{4}} \rightarrow 0, \quad \text { as } k \rightarrow \infty .
$$

In view of (5.40), this disproves the observability inequality

$$
\left(\mathcal{G}_{T} H, H\right)_{L_{+}^{2}\left(\Omega ; \mathbb{C}^{4}\right) \times \mathbb{C}^{4}} \geq C\|H\|_{L_{+}^{2}\left(\Omega ; \mathbb{C}^{4}\right) \times \mathbb{C}^{4}}^{2}-C^{\prime}\|H\|_{H_{+}^{-1}\left(\Omega ; \mathbb{C}^{4}\right) \times \mathbb{C}^{4}}^{2}, \quad \text { for all } H \in L_{+}^{2}\left(\Omega ; \mathbb{C}^{4}\right) \times \mathbb{C}^{4} .
$$

Recalling Theorem 5.5, this concludes the proof of Corollary 5.8. 


\section{Coupled waves with different speeds}

Here we consider a constant coefficient $\gamma>0, \gamma \neq 1$ and the following system

$$
\begin{cases}P u_{1}+b(x) u_{2}=0 & \text { in }(0, T) \times \Omega \\ P_{\gamma} u_{2}=b_{\omega}(x) f & \text { in }(0, T) \times \Omega\end{cases}
$$

with $P=\partial_{t}^{2}-\Delta$ as in the previous sections and $P_{\gamma}=\partial_{t}^{2}-\gamma^{2} \Delta$. The results of this section provide a proof for Theorem 1.5.

Our first result is a microlocal "hidden regularity" result. We prove that the uncontrolled solution $u_{1}$ of (6.1) enjoys more smoothness than expected.

Theorem 6.1. Let $s \in \mathbb{R}$. Assume that we have $\left(u_{1}^{0}, u_{1}^{1}\right) \in H^{s+3}(\Omega) \times H^{s+2}(\Omega),\left(u_{2}^{0}, u_{2}^{1}\right) \in H^{s+1}(\Omega) \times$ $H^{s}(\Omega)$ and $F \in L^{1}\left(0, T ; H^{s}(\Omega)\right)$. Then, the solution to System

$$
\begin{cases}P u_{1}+b(x) u_{2}=0 & \text { in } \mathbb{R} \times \Omega, \\ P_{\gamma} u_{2}=F & \text { in } \mathbb{R} \times \Omega,\end{cases}
$$

with the initial conditions,

$$
\left.\left(u_{1}, \partial_{t} u_{1}\right)\right|_{t=0}=\left(u_{1}^{0}, u_{1}^{1}\right),\left.\quad\left(u_{2}, \partial_{t} u_{2}\right)\right|_{t=0}=\left(u_{2}^{0}, u_{2}^{1}\right)
$$

satisfies the additional regularity $u_{1} \in \bigcap_{k=0}^{3} \mathscr{C}^{k}\left(\mathbb{R} ; H^{s+3-k}(\Omega)\right)$. Moreover, for all $T>0$ there exists a constant $C>0$ such that

$$
\begin{aligned}
& \sum_{k=0}^{3}\left\|\partial_{t}^{k} u_{1}\right\|_{L^{\infty}\left(0, T ; H^{s+3-k}(\Omega)\right)}+\left\|u_{2}\right\|_{L^{\infty}\left(0, T ; H^{s+1}(\Omega)\right)}+\left\|\partial_{t} u_{2}\right\|_{L^{\infty}\left(0, T ; H^{s}(\Omega)\right)} \\
& \leq C\left(\left\|u_{1}^{0}\right\|_{H^{s+3}(\Omega)}+\left\|u_{1}^{1}\right\|_{H^{s+2}(\Omega)}+\left\|u_{2}^{0}\right\|_{H^{s+1}(\Omega)}+\left\|u_{2}^{1}\right\|_{H^{s}(\Omega)}+\|F\|_{L^{1}\left(0, T ; H^{s}(\Omega)\right)}\right) \\
& \leq
\end{aligned}
$$

As a consequence, starting for instance from zero initial data and using $L^{2}$ controls, we cannot hope to reach any target in $\left(u_{1}(T), \partial_{t} u_{1}(T), u_{2}(T), \partial_{t} u_{2}(T)\right) \in\left(H^{2}(\Omega) \backslash H^{3}(\Omega)\right) \times\left(H^{1}(\Omega) \backslash H^{2}(\Omega)\right) \times$ $H^{1}(\Omega) \times L^{2}(\Omega)$. This proves the first item of Theorem 1.5.

Proof. First, we split the second equation in System (6.2) into

$$
\left\{\begin{array}{l}
P_{\gamma} \Pi_{+} u_{2}=\Pi_{+} F \\
\left.\left(\Pi_{+} u_{2}, \partial_{t} \Pi_{+} u_{2}\right)\right|_{t=0}=\left(\Pi_{+} u_{2}^{0}, \Pi_{+} u_{2}^{1}\right) \in H_{+}^{s+1}(\Omega) \times H_{+}^{s}(\Omega)
\end{array} \quad \text { in } \mathbb{R} \times \Omega\right.
$$

and the null-frequency part

$$
\begin{cases}P_{\gamma} \Pi_{0} u_{2}=\Pi_{0} F & \text { in } \mathbb{R} \times \Omega, \\ \left.\left(\Pi_{0} u_{2}, \partial_{t} \Pi_{0} u_{2}\right)\right|_{t=0}=\left(\Pi_{0} u_{2}^{0}, \Pi_{0} u_{2}^{1}\right) \in \mathbb{C}^{2} & \end{cases}
$$

Since $P_{\gamma} \Pi_{0} u_{2}=\partial_{t}^{2} \Pi_{0} u_{2}$, we have explicitely

$$
\Pi_{0} u_{2}(t)=\Pi_{0} u_{2}^{0}+t \Pi_{0} u_{2}^{1}+\int_{0}^{t}(t-s) \Pi_{0} F(s) d s,
$$

and hence $\Pi_{0} u_{2} \in \mathscr{C}^{1}(\mathbb{R} ; \mathbb{C})$. Similarly, we decompose $u_{1}$ as $u_{1}=\Pi_{0} u_{1}+\Pi_{+} u_{1}$. We have

$$
\left\{\begin{array}{l}
P \Pi_{0} u_{1}=-\Pi_{0} b u_{2} \\
\left.\left(\Pi_{0} u_{1}, \partial_{t} \Pi_{0} u_{1}\right)\right|_{t=0}=\left(\Pi_{0} u_{1}^{0}, \Pi_{0} u_{1}^{1}\right) \in \mathbb{C}^{2} .
\end{array} \quad \text { in } \mathbb{R} \times \Omega,\right.
$$


As $u_{2} \in \mathscr{C}^{1}\left(\mathbb{R} ; H^{s}(\Omega)\right)$, we obtain $\partial_{t}^{2} \Pi_{0} u_{1}=-\Pi_{0} b u_{2} \in \mathscr{C}^{1}(\mathbb{R} ; \mathbb{C})$, and hence $\Pi_{0} u_{1} \in \mathscr{C}^{3}(\mathbb{R} ; \mathbb{C})$. We split again $\Pi_{+} u_{1}$ into $\Pi_{+} u_{1}=v_{1}+w_{1}$, with

$$
\begin{cases}P v_{1}=-\Pi_{+} b \Pi_{0} u_{2} & \text { in } \mathbb{R} \times \Omega, \\ \left.\left(v_{1}, \partial_{t} v_{1}\right)\right|_{t=0}=\left(\Pi_{+} u_{1}^{0}, \Pi_{+} u_{1}^{1}\right) \in H_{+}^{s+3}(\Omega) \times H_{+}^{s+2}(\Omega), & \end{cases}
$$

and

$$
\left\{\begin{array}{l}
P w_{1}=-\Pi_{+} b \Pi_{+} u_{2} \\
\left.\left(w_{1}, \partial_{t} w_{1}\right)\right|_{t=0}=(0,0) .
\end{array} \quad \text { in } \mathbb{R} \times \Omega,\right.
$$

We directly have $v_{1} \in \mathscr{C}^{0}\left(\mathbb{R} ; H_{+}^{s+3}(\Omega)\right) \cap \mathscr{C}^{1}\left(\mathbb{R} ; H_{+}^{s+2}(\Omega)\right)$, since $\Pi_{+} b \Pi_{0} u_{2} \in \mathscr{C}^{0}\left(\mathbb{R} ; H_{+}^{k}(\Omega)\right)$ for all $k \in \mathbb{N}$.

We now focus on $w_{1}=\Pi_{+} w_{1}$. We use the splitting introduced in Section 5.3.2

$$
h_{+}=\frac{1}{2}\left(\Pi_{+} u_{2}^{0}-i \lambda^{-1} \Pi_{+} u_{2}^{1}\right) \in H_{+}^{s+1}(\Omega), \quad h_{-}=\frac{1}{2}\left(\Pi_{+} u_{2}^{0}+i \lambda^{-1} \Pi_{+} u_{2}^{1}\right) \in H_{+}^{s+1}(\Omega),
$$

so that we have the explicit Duhamel formula for $\Pi_{+} u_{2}$

$$
\begin{aligned}
\Pi_{+} u_{2}(s) & =e^{i s \gamma \lambda} h_{+}+e^{-i s \gamma \lambda} h_{-}+\frac{1}{2 i}(\gamma \lambda)^{-1} \int_{0}^{s}\left(e^{i(s-\sigma) \gamma \lambda}-e^{-i(s-\sigma) \gamma \lambda}\right) \Pi_{+} F(\sigma) d \sigma \\
& =e^{i s \gamma \lambda}\left(h_{+}+F_{+}(s)\right)+e^{-i s \gamma \lambda}\left(h_{-}+F_{-}(s)\right),
\end{aligned}
$$

where

$$
\begin{array}{ll}
F_{+}(s)=\frac{1}{2 i}(\gamma \lambda)^{-1} \int_{0}^{s} e^{-i \sigma \gamma \lambda} \Pi_{+} F(\sigma) d \sigma & \in \mathscr{C}^{0}\left(\mathbb{R} ; H_{+}^{s+1}(\Omega)\right), \\
F_{-}(s)=-\frac{1}{2 i}(\gamma \lambda)^{-1} \int_{0}^{s} e^{i \sigma \gamma \lambda} \Pi_{+} F(\sigma) d \sigma & \in \mathscr{C}^{0}\left(\mathbb{R} ; H_{+}^{s+1}(\Omega)\right) .
\end{array}
$$

The Duhamel formula for (6.5) gives

$$
\begin{aligned}
2 i \lambda w_{1}(t)=- & \int_{0}^{t}\left(e^{i(t-s) \lambda}-e^{-i(t-s) \lambda}\right) \Pi_{+} b \Pi_{+} u_{2}(s) d s \\
=- & e^{i t \lambda} \int_{0}^{t} e^{-i s \lambda} \Pi_{+} b\left(e^{i s \gamma \lambda}\left(h_{+}+F_{+}(s)\right)+e^{-i s \gamma \lambda}\left(h_{-}+F_{-}(s)\right)\right) d s \\
& +e^{-i t \lambda} \int_{0}^{t} e^{i s \lambda} \Pi_{+} b\left(e^{i s \gamma \lambda}\left(h_{+}+F_{+}(s)\right)+e^{-i s \gamma \lambda}\left(h_{-}+F_{-}(s)\right)\right) d s \\
=- & e^{i t \lambda} \int_{0}^{t} e^{-i s \lambda} \Pi_{+} b e^{i s \gamma \lambda} d s h_{+}-e^{i t \lambda} \int_{0}^{t} e^{-i s \lambda} \Pi_{+} b e^{i s \gamma \lambda} F_{+}(s) d s \\
& -e^{i t \lambda} \int_{0}^{t} e^{-i s \lambda} \Pi_{+} b e^{-i s \gamma \lambda} d s h_{-}-e^{i t \lambda} \int_{0}^{t} e^{-i s \lambda} \Pi_{+} b e^{-i s \gamma \lambda} F_{-}(s) d s \\
& +e^{-i t \lambda} \int_{0}^{t} e^{i s \lambda} \Pi_{+} b e^{i s \gamma \lambda} d s h_{+}+e^{-i t \lambda} \int_{0}^{t} e^{i s \lambda} \Pi_{+} b e^{i s \gamma \lambda} F_{+}(s) d s \\
& +e^{-i t \lambda} \int_{0}^{t} e^{i s \lambda} \Pi_{+} b e^{-i s \gamma \lambda} d s h_{-}+e^{-i t \lambda} \int_{0}^{t} e^{i s \lambda} \Pi_{+} b e^{-i s \gamma \lambda} F_{-}(s) d s .
\end{aligned}
$$

The data $h_{ \pm}$are in $H_{+}^{s+1}(\Omega)$, therefore, using Lemma A.1 (with $\Pi_{+} b \in \Psi_{\mathrm{phg}}^{0}(\Omega)$ ), all the corresponding terms above belong to $\mathscr{C}^{0}\left(\mathbb{R} ; H_{+}^{s+2}(\Omega)\right.$ ). Similarly, using Lemma A.3, all terms including $F_{ \pm}$above also belong to $\mathscr{C}^{0}\left(\mathbb{R} ; H_{+}^{s+2}(\Omega)\right)$. As a consequence, we have $w_{1} \in \mathscr{C}^{0}\left(\mathbb{R} ; H_{+}^{s+3}(\Omega)\right)$. Differentiating with respect to time expression (6.6) and using again lemmata A.1 and A.3, we obtain $\partial_{t} w_{1} \in \mathscr{C}^{0}\left(\mathbb{R} ; H_{+}^{s+2}(\Omega)\right)$. 
Recalling that $u_{1}=\Pi_{0} u_{1}+v_{1}+w_{1}$ and using the regularity properties of each of these three terms, we obtain $u_{1} \in \mathscr{C}^{0}\left(\mathbb{R} ; H^{s+3}(\Omega)\right) \cap \mathscr{C}^{1}\left(\mathbb{R} ; H^{s+2}(\Omega)\right)$. Coming back to the equation

$$
\partial_{t}^{2} u_{1}=\Delta u_{1}-b u_{2} \in \mathscr{C}^{0}\left(\mathbb{R} ; H^{s+1}(\Omega)\right) \cap \mathscr{C}^{1}\left(\mathbb{R} ; H^{s}(\Omega)\right),
$$

we finally obtain $u_{1} \in \bigcap_{k=0}^{3} \mathscr{C}^{k}\left(\mathbb{R} ; H^{s+3-k}(\Omega)\right)$.

Estimate (6.3) comes from the estimates of lemmata A.1 and A.3 applied to all terms in (6.6).

The next result shows that the situation becomes worse if $\bar{\omega} \cap \overline{\mathcal{O}}$ does not satisfy GCC. In this case one cannot hope to control in any Sobolev space.

Theorem 6.2. Assume that $\bar{\omega} \cap \overline{\mathcal{O}}$ does not satisfy GCC. Then, for all $s \geq 0$, there exists $\left(u_{1}^{0}, u_{1}^{1}\right) \in$ $H^{s+1}(\Omega) \times H^{s}(\Omega)$, such that the solution to System (6.1) for all $T>0$ and all $f \in L^{2}((0, T) \times \Omega)$, along with the following initial conditions,

$$
\left.\left(u_{1}, \partial_{t} u_{1}\right)\right|_{t=0}=\left(u_{1}^{0}, u_{1}^{1}\right),\left.\quad\left(u_{2}, \partial_{t} u_{2}\right)\right|_{t=0}=(0,0)
$$

satisfies

$$
\left(u_{1}(T), \partial_{t} u_{1}(T), u_{2}(T), \partial_{t} u_{2}(T)\right) \neq(0,0,0,0) .
$$

Remark 6.3. 1. This result proves the second item of Theorem 1.5.

2. The result stated above can be improved in many ways. The case of two different metrics, $g_{1}$ and $g_{2}$ can also be addressed with the ideas of the proof, assuming only that $\eta \mapsto\left(g_{1}-g_{2}\right)_{x}(\eta, \eta)$ is a nondegenerate quadratic form everywhere on $\Omega$. Here we restrict ourselves to the simple situation $g_{2}=\gamma^{2} g_{1}, \gamma \neq 1$ for the sake of exposition.

Proof of Theorem 6.2. We recall that the operators $L_{+}, L_{-}$are defined in Section 2.1.

As $\bar{\omega} \cap \overline{\mathcal{O}}$ does not satisfy GCC there exists a bicharacterisitics $\Gamma$ that never meets $\pi^{-1}(\bar{\omega} \cap \overline{\mathcal{O}})$ in $T^{*} M$. Without any loss of generality let us assume that $\Gamma \subset$ Char $L_{+}$.

We have

$$
P_{\gamma} u_{2}=b_{\omega}(x) f,\left.\quad\left(u_{2}, \partial_{t} u_{2}\right)\right|_{t=0}=(0,0) .
$$

Extending $f$ by zero outside $[0, T]$ we have $\operatorname{supp}\left(u_{2}\right) \subset\{t \geq 0\}$. For $u_{1}^{0}$ and $u_{1}^{1}$ to be defined below we introduce $w$ and $v$ in the following way:

$$
\begin{aligned}
& P v=-b(x) u_{2},\left.\quad\left(v, \partial_{t} v\right)\right|_{t=0}=(0,0), \\
& P w=0,\left.\quad\left(w, \partial_{t} w\right)\right|_{t=0}=\left(u_{1}^{0}, u_{1}^{1}\right) .
\end{aligned}
$$

The linearity of the equations yields $u_{1}=v+w$.

Study of $\boldsymbol{w}$. Since $P$ is elliptic at $(t, x, 1,0)$ we have

$$
(t, x, 1,0) \notin \mathrm{WF}(w) \quad \forall t, x .
$$

Let $\rho_{0}=\left(0, x_{0}, \tau_{0}, \eta_{0}\right) \in \Gamma \cap\{t=0\}$. We choose $u_{1}^{0} \in H^{s+1}(\Omega)$ such that $\left\{\left(x_{0}, \mathbb{R}_{+}^{*} \eta_{0}\right)\right\}=$ $\mathrm{WF}\left(u_{1}^{0}\right)$. We then set $u_{1}^{1}=i \lambda u_{1}^{0} \in H^{s}(\Omega)$.

We set $z=L_{+} w$ and because of the precise choice we made for $\left(u_{1}^{0}, u_{1}^{1}\right)$ we observe that $\left.z\right|_{t=0}=0$. It follows that

$$
L_{-} z=-P w=0,\left.\quad z\right|_{t=0}=0
$$

\footnotetext{
${ }^{4}$ To choose $u_{1}^{0}$ we can invoque the constructive approach of Theorem 8.1.4 in [Hör94] that yields at first a distribution with $\left\{\left(x_{0}, \mathbb{R}_{+}^{*} \eta_{0}\right)\right\}$ for wavefront set. Since $\Omega$ is compact, this distribution has finite order and belongs to $H^{\sigma}(\Omega)$ for some $\sigma \in \mathbb{R}$, by the Paley-Wiener theorem. Finally, we can apply an appropriate power of the Laplace operator, an elliptic pseudo-differential operator on $\Omega$ (according to [See67] or [Shu01, Theorem 11.2]) that preserves the wavefront set, to yield the proper function in the Sobolev space $H^{s+1}(\Omega)$.
} 
which gives $z=0$ (see e.g. Theorem 23.1.2 in [Hör85]) and therefore

$$
L_{+} w=0,\left.\quad w\right|_{t=0}=u_{1}^{0} .
$$

In a local chart we consider $\chi(\tau, \eta)$ a symbol of order 0 with support in $V$ and equal to one in $V^{\prime}$, for $V^{\prime} \Subset V$ neighborhoods of $\{\eta=0\}$. Then $(1-\mathrm{op}(\chi)) L_{+}$is a pseudo-differential operator of order one in all variables [Hör85, Theorem 18.1.35].

We have $(1-\mathrm{op}(\chi)) L_{+} w=0$ which, by (6.7) and choosing $V$ sufficiently small, implies

$$
\mathrm{WF}(w) \subset \operatorname{Char}\left(L_{+}\right) .
$$

Since $\left(x_{0}, \eta_{0}\right) \in \mathrm{WF}\left(\left.w\right|_{t=0}\right)$, by Theorem 8.2.4 in [Hör90] we have that $\left(0, x_{0}, \tau, \eta_{0}\right) \in \operatorname{WF}(w)$ for some $\tau \in \mathbb{R}$ and necessarily $\tau=\tau_{0}$, that is, $\rho_{0} \in \mathrm{WF}(w)$. Since $P w=0$, the singularity propagation theorem of Hörmander [Hör94, Theorem 26.1.1] implies that

$$
\Gamma \subset \mathrm{WF}(w) .
$$

Study of $\boldsymbol{v} . \quad A s \operatorname{supp}\left(b u_{2}\right) \subset\{t \geq 0\}$ we have

$$
v=0 \text { in }\{t \leq 0\} .
$$

From $P_{\gamma} u_{2}=b_{\omega}(x) f$, we have $\mathrm{WF}\left(u_{2}\right) \subset \operatorname{Char}\left(P_{\gamma}\right) \cup \mathrm{WF}\left(b_{\omega}(x) f\right)$ by Theorem 18.1.28 in [Hör85]. As $\Gamma$ does not meet $\pi^{-1}(\bar{\omega} \cap \overline{\mathcal{O}})$ and $\Gamma \cap \operatorname{Char}\left(P_{\gamma}\right)=\emptyset$ we find

$$
\Gamma \cap \mathrm{WF}\left(b u_{2}\right) \subset \Gamma \cap \pi^{-1}(\overline{\mathcal{O}}) \cap \pi^{-1}(\bar{\omega})=\emptyset .
$$

The singularity propagation theorem of Hörmander [Hör94, Theorem 26.1.1] implies that $\operatorname{WF}(v) \backslash$ $\mathrm{WF}\left(b u_{2}\right)$ is invariant by the hamiltonian vector field $H_{p}$. Since $\Gamma$ is invariant by $H_{p}$ and with (6.10) and (6.9) we obtain that

$$
\Gamma \cap \operatorname{WF}(v)=\emptyset
$$

Conclusion. From (6.8) and (6.11) we find that $\Gamma \subset \mathrm{WF}\left(u_{1}\right)$. It follows that $\left(u_{1}, \partial_{t} u_{1}, u_{2}, \partial_{t} u_{2}\right)$ cannot vanish at the final control time $T$. In fact it would first imply $u_{2}=0$ in $\{t \geq T\}$ where it satisfies $P_{\gamma} u_{2}=0$. Second, we would have $P u_{1}=0$ in $\{t \geq T\}$ implying $u_{1}=0$ in $\{t \geq T\}$, which obviously does not hold.

To conclude the study of System (6.1), we prove an "almost converse" of the last two Theorems 6.1 and 6.2. We prove the controllability of System (6.1) in the space $\left(H^{3}(\Omega) \times H^{2}(\Omega)\right) \times\left(H^{1}(\Omega) \times L^{2}(\Omega)\right)$, if $\omega \cap \mathcal{O}$ satisfies GCC.

Theorem 6.4. Assume that $\omega \cap \mathcal{O}$ satisfies $G C C$ and that $T>\max \left\{T_{\omega \cap \mathcal{O}}(1), T_{\omega}(\gamma)\right\}$. Then, System (6.1) is controllable in the space $H^{3}(\Omega) \times H^{2}(\Omega)$ in time $T$ in the sense given in Section 1.2.3.

Remark that $T_{\omega}(\gamma)$ is the time needed to control the second component of System (6.1), which is directly controlled, whereas $T_{\omega \cap \mathcal{O}}(1)$ is the time needed to control the first component of System (6.1).

Note also that for $T<T_{\omega}(\gamma)$, the second equation is not controllable. Similarly, if $T<T_{\bar{\omega} \cap \overline{\mathcal{O}}}(1)$ the first equation is not controllable: one can find a bicharacteristic curve $\Gamma$ that does not meet $\pi^{-1}(\bar{\omega} \cap \overline{\mathcal{O}})$ in the time interval $[0, T]$ and the same construction as in the proof of Theorem 6.2 applies. We thus obtain the third item of Theorem 1.5.

Proof. According to Proposition 5.1 (case $s=2, \sigma=0$ ) and Theorem 6.1, the result is equivalent to proving the observability inequality

$$
E_{-2}\left(v_{1}(0)\right)+E_{0}\left(v_{2}(0)\right) \leq C \int_{0}^{T}\left\|b_{\omega} v_{2}\right\|_{L^{2}(\Omega)}^{2} d t,
$$


for all $\left(v_{1}, v_{2}\right) \in\left(\mathscr{C}^{0}\left(\mathbb{R} ; H^{-2}(\Omega)\right) \cap \mathscr{C}^{1}\left(\mathbb{R} ; H^{-3}(\Omega)\right)\right) \times\left(\mathscr{C}^{0}\left(\mathbb{R} ; L^{2}(\Omega)\right) \cap \mathscr{C}^{1}\left(\mathbb{R} ; H^{-1}(\Omega)\right)\right)$ solutions of (5.3). Setting $w_{1}=(1-\Delta)^{-1} v_{1}, w_{2}=v_{2}$, this observability inequality is equivalent to proving

$$
E_{0}\left(w_{1}(0)\right)+E_{0}\left(w_{2}(0)\right) \leq C \int_{0}^{T}\left\|b_{\omega} w_{2}\right\|_{L^{2}(\Omega)}^{2} d t,
$$

for all $\left(w_{1}, w_{2}\right) \in \mathscr{C}^{0}\left(\mathbb{R} ; L^{2}\left(\Omega ; \mathbb{C}^{2}\right)\right) \cap \mathscr{C}^{1}\left(\mathbb{R} ; H^{-1}\left(\Omega ; \mathbb{C}^{2}\right)\right)$ solutions to

$$
\begin{cases}P w_{1}=0 & \text { in }(0, T) \times \Omega, \\ P_{\gamma} w_{2}=-b(x)(1-\Delta) w_{1} & \text { in }(0, T) \times \Omega .\end{cases}
$$

Recall that System (6.14) is well-posed in this space, according to Theorem 6.1. As in Section 3, we prove (6.13) in two steps with a compactness-uniqueness strategy. The first step is to prove the relaxed observability inequality

$$
E_{0}\left(w_{1}(0)\right)+E_{0}\left(w_{2}(0)\right) \leq C\left(\int_{0}^{T} \int_{\omega}\left|b_{\omega} w_{2}\right|^{2} d x d t+E_{-1}\left(w_{1}(0)\right)+E_{-1}\left(w_{2}(0)\right)\right),
$$

for all $\left(w_{1}, w_{2}\right) \in \mathscr{C}^{0}\left(\mathbb{R} ; L^{2}\left(\Omega ; \mathbb{C}^{2}\right)\right) \cap \mathscr{C}^{1}\left(\mathbb{R} ; H^{-1}\left(\Omega ; \mathbb{C}^{2}\right)\right)$ solutions to $(6.14)$.

We proceed by contradiction and suppose that the observability inequality (6.15) is not satisfied. Thus, there exists a sequence $\left(w_{1}^{k}, w_{2}^{k}\right)_{k \in \mathbb{N}}$ of $\mathscr{C}^{0}\left(0, T ; L^{2}(\Omega)\right) \cap \mathscr{C}^{1}\left(0, T ; H^{-1}(\Omega)\right)$-solutions of $(6.14)$ such that

$$
\begin{aligned}
& E_{0}\left(w_{1}^{k}(0)\right)+E_{0}\left(w_{2}^{k}(0)\right)=1, \\
& \int_{0}^{T} \int_{\omega}\left|b_{\omega} w_{2}^{k}\right|^{2} d x d t \rightarrow 0, \quad k \rightarrow \infty, \\
& E_{-1}\left(w_{1}^{k}(0)\right)+E_{-1}\left(w_{2}^{k}(0)\right) \rightarrow 0, \quad k \rightarrow \infty .
\end{aligned}
$$

According to (6.16) and to (6.18), together with the continuity of the solution with respect to the initial data given by Theorem 6.1 , the sequence $\left(w_{1}^{k}, w_{2}^{k}\right)$ is bounded in $L^{2}\left(M_{T} ; \mathbb{C}^{2}\right)$, and converges to zero in $H^{-1}\left(M_{T} ; \mathbb{C}^{2}\right)$. It follows that

$$
\left(w_{1}^{k}, w_{2}^{k}\right) \rightarrow(0,0) \quad \text { in } L^{2}\left(M_{T} ; \mathbb{C}^{2}\right) .
$$

As a consequence of [Gér91, Theorem 1], there exists a subsequence of $\left(w_{1}^{k}, w_{2}^{k}\right)_{k \in \mathbb{N}}$ (still denoted $\left(w_{1}^{k}, w_{2}^{k}\right)_{k \in \mathbb{N}}$ in what follows) and two associated microlocal defect measures

$$
\mu_{1} \in \mathcal{M}_{+}\left(S^{*} M_{T}\right), \quad \mu_{2} \in \mathcal{M}_{+}\left(S^{*} M_{T}\right),
$$

such that for any $A \in \Psi_{\mathrm{phg}}^{0}\left(M_{T}\right)$,

$$
\lim _{k \rightarrow \infty}\left(A w_{1}^{k}, w_{1}^{k}\right)_{L^{2}\left(M_{T} ; \mathbb{C}\right)}=\left\langle\mu_{1}, \sigma_{0}(A)\right\rangle_{S^{*} M_{T}}, \quad \lim _{k \rightarrow \infty}\left(A w_{2}^{k}, w_{2}^{k}\right)_{L^{2}\left(M_{T} ; \mathbb{C}\right)}=\left\langle\mu_{2}, \sigma_{0}(A)\right\rangle_{S^{*} M_{T}} .
$$

Note that in this case (as opposed to the case $\gamma=1$ treated in Section 3) we shall not use the coupling of the two waves and of the associated measure $\mu_{12}$.

The first equation of (6.14) yields, as in Lemma 3.3 that

$$
\operatorname{supp}\left(\mu_{1}\right) \subset \operatorname{Char}(P), \quad \text { and } \quad\left\langle\mu_{1}, H_{p} a\right\rangle_{S^{*} M_{T}}=0,
$$

for any $a \in S_{\text {phg }}^{-1}\left(T^{*} M_{T} ; \mathbb{C}\right)$. Moreover, $(6.17)$ gives

$$
\operatorname{supp}\left(\mu_{2}\right) \cap \pi^{-1}((0, T) \times \omega)=\emptyset .
$$

This gives, for any $\chi \in \mathscr{C}_{c}^{\infty}((0, T) \times(\mathcal{O} \cap \omega))$,

$$
\left((1-\Delta)^{-2} \chi P_{\gamma} w_{2}^{k}, \chi P_{\gamma} w_{2}^{k}\right)_{L^{2}\left(M_{T} ; \mathbb{C}\right)} \rightarrow\left\langle\mu_{2}, p_{\gamma}^{2}|\eta|_{x}^{-4} \chi^{2}\right\rangle_{S^{*} M_{T}}=0 .
$$


Using the second equation of (6.14), we now have

$$
0=\lim _{k \rightarrow+\infty}\left((1-\Delta)^{-2} \chi b(1-\Delta) w_{1}^{k}, \chi b(1-\Delta) w_{1}^{k}\right)_{L^{2}\left(M_{T} ; \mathbb{C}\right)}=\left\langle\mu_{1}, \chi^{2} b^{2}\right\rangle_{S^{*} M_{T}} .
$$

As a consequence, we have

$$
\operatorname{supp}\left(\mu_{1}\right) \cap \pi^{-1}((0, T) \times \mathcal{O} \cap \omega)=\emptyset .
$$

This, together with the free propagation of $\mu_{1}$ given in (6.19), and the assumption that $\mathcal{O} \cap \omega$ satisfies GCC, implies that $\mu_{1}$ vanishes identically on $(0, T) \times \Omega$, as soon as $T>T_{\omega \cap \mathcal{O}}(1)$. Hence, we have $w_{1}^{k} \rightarrow 0$ in $L_{\mathrm{loc}}^{2}\left(M_{T}\right)$.

It remains to study $w_{2}^{k}$. We have $P_{\gamma} w_{2}^{k}=-b(x)(1-\Delta) w_{1}^{k} \rightarrow 0$ in $H_{\mathrm{loc}}^{-2}\left(M_{T}\right)$ since $w_{1}^{k} \rightarrow 0$ $L_{\text {loc }}^{2}\left(M_{T}\right)$. This yields $\operatorname{supp}\left(\mu_{2}\right) \subset \operatorname{Char}\left(P_{\gamma}\right)$.

Now, take $\chi_{1} \in S_{\mathrm{phg}}^{0}\left(M_{T}\right), 0 \leq \chi_{1}$, such that

- $\chi_{1}=1$ in a neighborhood of $\operatorname{Char}\left(P_{\gamma}\right)$,

- $\chi_{1}=0$ in a neighborhood of $\operatorname{Char}(P)$,

and let $\Xi_{1} \in \Psi_{\mathrm{phg}}^{0}\left(M_{T}\right)$ an operator such that $\sigma_{0}\left(\Xi_{1}\right)=\chi_{1}$. There exists a parametrix $Q \in \Psi_{\mathrm{phg}}^{-2}\left(M_{T}\right)$ such that (see for instance [Hör85, Theorem 18.1.24]),

$$
Q P=\Xi_{1}+R, \quad R \in \Psi_{\mathrm{phg}}^{-\infty}\left(M_{T}\right) .
$$

Applying this parametrix to the first equation of (6.14) gives

$$
\Xi_{1} w_{1}^{k}+R w_{1}^{k}=0
$$

In particular, we have $\Xi_{1} w_{1}^{k} \rightarrow 0$ in $H_{\text {loc }}^{s}\left(M_{T}\right)$ for all $s \in \mathbb{R}$.

Consider now $\chi_{2}$ satisfying the same properties as $\chi_{1}$ with moreover $\chi_{1}=1$ on a neighborhood of $\operatorname{supp}\left(\chi_{2}\right)$. Let $\Xi_{2} \in \Psi_{\mathrm{phg}}^{0}\left(M_{T}\right)$ an operator such that $\sigma_{0}\left(\Xi_{2}\right)=\chi_{2}$.

Writing $w_{2}^{k}=\left(1-\Xi_{2}\right) w_{2}^{k}+\Xi_{2} w_{2}^{k}$, we directly have $\left(1-\Xi_{2}\right) w_{2}^{k} \rightarrow 0$ in $L_{\text {loc }}^{2}\left(M_{T}\right)$ as $\operatorname{supp}\left(\mu_{2}\right) \subset$ Char $\left(P_{\gamma}\right)$. Below, we shall prove the following convergence.

Lemma 6.5. $P_{\gamma} \Xi_{2} w_{2}^{k} \rightarrow 0$ in $H_{\mathrm{loc}}^{-1}\left(M_{T}\right)$.

This implies that $\left\langle\mu_{2}, H_{p_{\gamma}} a\right\rangle_{S^{*} M_{T}}=\left\langle\mu_{2},\left|\chi_{2}\right|^{2} H_{p_{\gamma}} a\right\rangle_{S^{*} M_{T}}=0$ for any $a \in S_{\text {phg }}^{-1}\left(T^{*} M_{T}\right)$. Hence, the measure $\mu_{2}$ satisfies a free propagation relation along the bicharacteristic flow of $P_{\gamma}$. Since $\operatorname{supp}\left(\mu_{2}\right) \cap \pi^{-1}((0, T) \times \omega)=\emptyset$, the measure $\mu_{2}$ vanishes identically on $S^{*} M_{T}$ as soon as $T>$ $T_{\omega}(\gamma)$. This proves that $\left(w_{1}^{k}, w_{2}^{k}\right) \rightarrow(0,0)$ in $L_{\text {loc }}^{2}\left(M_{T} ; \mathbb{C}^{2}\right)$, and, following the end of the proof of Proposition 3.2, that $E_{0}\left(w_{1}^{k}(0)\right)+E_{0}\left(w_{2}^{k}(0)\right) \rightarrow 0$. This yields a contradiction with (6.16), and concludes the proof of the relaxed observability inequality (6.15).

We conclude the proof of (6.13) as in Section 3.2. We define the set of invisible solutions

$$
\begin{gathered}
\mathcal{N}(T)=\left\{\mathscr{W}=\left(w_{1}^{0}, w_{2}^{0}, w_{1}^{1}, w_{2}^{1}\right) \in \mathscr{H} \text { such that the associated solution of }(6.14)\right. \\
\text { satisfies } \left.w_{2}(t, x)=0 \text { for all }(t, x) \in(0, T) \times \omega\right\},
\end{gathered}
$$

As in Section 3.2, proving that $\mathcal{N}(T)=\{0\}$ implies that (6.13) is true. Again, proving that $\mathcal{N}(T)=$ $\{0\}$ is equivalent to proving that there is no eigenfunction $\left(\varphi_{1}, \varphi_{2}\right)$ of the operator

$$
\left(\begin{array}{cc}
-\Delta & 0 \\
b(x)(1-\Delta) & -\gamma^{2} \Delta
\end{array}\right)
$$

such that $\left.\varphi_{2}\right|_{\omega}=0$. Indeed, letting $\mu$ be the associated eigenvalue, we have

$$
-\gamma^{2} \Delta \varphi_{2}+b(x)(1-\Delta) \varphi_{1}=\mu \varphi_{2}, \quad \text { and }\left.\quad \varphi_{2}\right|_{\mathcal{O} \cap \omega}=0 .
$$


This yields in particular $\left.(1-\Delta) \varphi_{1}\right|_{\mathcal{O} \cap \omega}=0$. As $-\Delta \varphi_{1}=\mu \varphi_{1}$, the function $(1-\Delta) \varphi_{1}$ is an eigenfunction of $-\Delta$, vanishing on the nonempty open set $\mathcal{O} \cap \omega$, and thus vanishes identically (see for instance [Aro57, AKS62, LL11]). Hence, we have $\varphi_{1}=0$. Coming back to (6.21), and using the same argument, we obtain $\varphi_{2}=0$. Finally, this proves that $\mathcal{N}(T)=\{0\}$.

Note that a quantitative version of this unique continuation result is proved in [Léa10, Proposition 5.1]. This concludes the proof of the observability inequality (6.13), and hence that of Theorem 6.4.

Proof of Lemma 6.5. We have

$$
P_{\gamma} \Xi_{2} w_{2}^{k}=\Xi_{2} P_{\gamma} w_{2}^{k}+\left[P_{\gamma}, \Xi_{2}\right] w_{2}^{k}=-\Xi_{2} b(x)(1-\Delta) w_{1}^{k}+\left[P_{\gamma}, \Xi_{2}\right] w_{2}^{k} .
$$

We write

$$
\Xi_{2} b(x)(1-\Delta) w_{1}^{k}=\Xi_{2} b(x)(1-\Delta) \Xi_{1} w_{1}^{k}+\Xi_{2} b(x)(1-\Delta)\left(1-\Xi_{1}\right) w_{1}^{k}
$$

Since the supports of $\chi_{2}$ and $1-\chi_{1}$ are disjoint the operator $\Xi_{2} b(x)(1-\Delta)\left(1-\Xi_{1}\right)$ is regularizing and since $\Xi_{1} w_{1}^{k} \rightarrow 0$ in $H_{\text {loc }}^{s}\left(M_{T}\right)$ for all $s \in \mathbb{R}$ then the same holds for $\Xi_{2} b(x)(1-\Delta) w_{1}^{k}$. For the second term in $(6.22)$ we write

$$
\left[P_{\gamma}, \Xi_{2}\right] w_{2}^{k}=\left[P_{\gamma}, \Xi_{2}\right] \Xi_{3} w_{2}^{k}+\left[P_{\gamma}, \Xi_{2}\right]\left(1-\Xi_{3}\right) w_{2}^{k},
$$

where $\Xi_{3} \in \Psi_{\mathrm{phg}}^{0}\left(M_{T}\right)$ is an operator such that $\sigma_{0}\left(\Xi_{3}\right)=\chi_{3}$ with $\chi_{3}$ satisfying the same properties as $\chi_{2}$ with moreover $\chi_{2}=1$ on a neighborhood of $\operatorname{supp}\left(\chi_{3}\right)$. We then have $\left[P_{\gamma}, \Xi_{2}\right] \Xi_{3}$ regularizing. Since $\left(1-\Xi_{3}\right) w_{2}^{k} \rightarrow 0$ in $L_{\text {loc }}^{2}\left(M_{T}\right)$ as $\operatorname{supp}\left(\mu_{2}\right) \subset \operatorname{Char}\left(P_{\gamma}\right)$ and $\left[P_{\gamma}, \Xi_{2}\right]$ is order one, we find that $\left[P_{\gamma}, \Xi_{2}\right] w_{2}^{k} \rightarrow 0$ in $H_{\mathrm{loc}}^{-1}\left(M_{T}\right)$. This concludes the proof.

\section{A 1-smoothing properties}

In this section, we state and prove three lemmata concerning the 1-smoothing properties of some families of operators. These properties are used in a crucial way in the proofs of Theorems 5.3, 5.5 and 6.1 .

The proofs given in this section are elementary and are inspired by [DL09]. These properties however deeply rely on the Fourier integral operator property of propagators of type $e^{i t \lambda}$.

Lemma A.1. Let $s \in \mathbb{R}, \gamma \neq \delta, b \in \Psi_{\mathrm{phg}}^{0}(\Omega)$. Then, the operator defined by

$$
A(t)=\int_{0}^{t} e^{-i z \gamma \lambda} b e^{i z \delta \lambda} d z
$$

satisfies $A \in \mathscr{C}^{0}\left(\mathbb{R} ; \mathcal{L}\left(H^{s}(\Omega), H^{s+1}(\Omega)\right)\right)$. In particular, for all $t \in \mathbb{R}, A(t)$ is 1-smoothing.

Lemma A.1 can be seen as a corollary of Lemma A.2, and we hence omit its proof. We chose to state Lemma A.1 separately since in the main part of the article, regularizing properties are most often used under this simpler form.

Lemma A.2. Let $s \in \mathbb{R}, \gamma \neq \delta, b \in \Psi_{\mathrm{phg}}^{0}(\Omega)$ and consider $m, \tilde{m} \in \mathscr{C}^{0}\left(\mathbb{R}, \Psi_{\mathrm{phg}}^{0}(\Omega)\right)$ two continuous families of operators. Then, the operator defined by

$$
A(t)=\int_{0}^{t}\left(\int_{0}^{z} m(\sigma) d \sigma\right) e^{-i z \gamma \lambda} b e^{i z \delta \lambda}\left(\int_{0}^{z} \tilde{m}(\sigma) d \sigma\right) d z
$$

satisfies $A \in \mathscr{C}^{0}\left(\mathbb{R} ; \mathcal{L}\left(H^{s}(\Omega), H^{s+1}(\Omega)\right)\right)$. In particular, for all $t \in \mathbb{R}, A(t)$ is 1-smoothing.

The next lemma is only used in the proof of Theorem 6.1 and is proved at the end of this section. 
Lemma A.3. Let $s \in \mathbb{R}, T>0 \gamma \neq \delta, b \in \Psi_{\mathrm{phg}}^{0}(\Omega)$, and suppose that $F \in L^{1}\left(0, T ; H^{s}(\Omega)\right)$. Then, the function defined by

$$
\mathscr{F}(t)=\int_{0}^{t} e^{-i z \gamma \lambda} b e^{i z \delta \lambda}\left(\int_{0}^{z} F(\sigma) d \sigma\right) d z
$$

satisfies $\mathscr{F} \in \mathscr{C}^{0}\left(0, T ; H^{s+1}(\Omega)\right)$, and

$$
\|\mathscr{F}\|_{L^{\infty}\left(0, T ; H^{s+1}\right)} \leq C\|F\|_{L^{1}\left(0, T ; H^{s}\right)},
$$

for some $C=C(s, T, \gamma, \delta, b)>0$.

Proof of Lemma A.2. We first notice that $A(t) \in \mathscr{C}^{0}\left(\mathbb{R} ; \mathcal{L}\left(H^{s}(\Omega)\right)\right)$ since all operators in $A(t)$ preserve the regularity. It suffices to prove that $\lambda A(t) \in \mathscr{C}^{0}\left(\mathbb{R} ; \mathcal{L}\left(H^{s}(\Omega)\right)\right)$. For this, we compute

$$
\begin{aligned}
-i \gamma \lambda A(t)= & -i \gamma \lambda \int_{0}^{t}\left(\int_{0}^{z} m(\sigma) d \sigma\right) e^{-i z \gamma \lambda} b e^{i z \delta \lambda}\left(\int_{0}^{z} \tilde{m}(\sigma) d \sigma\right) d z \\
= & \int_{0}^{t}\left(\int_{0}^{z} m(\sigma) d \sigma\right)(-i \gamma \lambda) e^{-i z \gamma \lambda} b e^{i z \delta \lambda}\left(\int_{0}^{z} \tilde{m}(\sigma) d \sigma\right) d z \\
& \quad+\int_{0}^{t}\left[(-i \gamma \lambda),\left(\int_{0}^{z} m(\sigma) d \sigma\right)\right] e^{-i z \gamma \lambda} b e^{i z \delta \lambda}\left(\int_{0}^{z} \tilde{m}(\sigma) d \sigma\right) d z,
\end{aligned}
$$

and we notice that the last term

$$
\int_{0}^{t}\left[(-i \gamma \lambda),\left(\int_{0}^{z} m(\sigma) d \sigma\right)\right] e^{-i z \gamma \lambda} b e^{i z \delta \lambda}\left(\int_{0}^{z} \tilde{m}(\sigma) d \sigma\right) d z \in \mathscr{C}^{0}\left(\mathbb{R} ; \mathcal{L}\left(H^{s}(\Omega), H^{s}(\Omega)\right)\right),
$$

since $\left[(-i \gamma \lambda),\left(\int_{0}^{z} m(\sigma) d \sigma\right)\right]=-i \gamma \int_{0}^{z}[\lambda, m(\sigma)] d \sigma \in \mathscr{C}^{0}\left(\mathbb{R}, \Psi_{\mathrm{phg}}^{0}(\Omega)\right)$. As a consequence, we can write, for some $R_{t} \in \mathscr{C}^{0}\left(\mathbb{R} ; \mathcal{L}\left(H^{s}(\Omega)\right)\right)$,

$$
-i \gamma \lambda A(t)=\int_{0}^{t}\left(\int_{0}^{z} m(\sigma) d \sigma\right) \partial_{z}\left(e^{-i z \gamma \lambda}\right) b e^{i z \delta \lambda}\left(\int_{0}^{z} \tilde{m}(\sigma) d \sigma\right) d z+R_{t} .
$$

After an integration by parts, this gives

$$
\begin{aligned}
-i \gamma \lambda A(t)= & -\int_{0}^{t}\left(\int_{0}^{z} m(\sigma) d \sigma\right) e^{-i z \gamma \lambda} b \partial_{z}\left(e^{i z \delta \lambda}\right)\left(\int_{0}^{z} \tilde{m}(\sigma) d \sigma\right) d z \\
& -\int_{0}^{t} m(z) e^{-i z \gamma \lambda} b e^{i z \delta \lambda}\left(\int_{0}^{z} \tilde{m}(\sigma) d \sigma\right) d z-\int_{0}^{t}\left(\int_{0}^{z} m(\sigma) d \sigma\right) e^{-i z \gamma \lambda} b e^{i z \delta \lambda} \tilde{m}(z) d z \\
& +\left[\left(\int_{0}^{z} m(\sigma) d \sigma\right) e^{-i z \gamma \lambda} b e^{i z \delta \lambda}\left(\int_{0}^{z} \tilde{m}(\sigma) d \sigma\right)\right]_{0}^{t}+R_{t} .
\end{aligned}
$$

Except the first term in the right hand-side of this expression, all terms are in $\mathscr{C}^{0}\left(\mathbb{R} ; \mathcal{L}\left(H^{s}(\Omega)\right)\right)$, so that we can write, for some $\tilde{R}_{t} \in \mathscr{C}^{0}\left(\mathbb{R} ; \mathcal{L}\left(H^{s}(\Omega)\right)\right)$,

$$
\begin{aligned}
-i \gamma \lambda A(t)= & -\int_{0}^{t}\left(\int_{0}^{z} m(\sigma) d \sigma\right) e^{-i z \gamma \lambda} b \partial_{z}\left(e^{i z \delta \lambda}\right)\left(\int_{0}^{z} \tilde{m}(\sigma) d \sigma\right) d z+\tilde{R}_{t} \\
= & -\int_{0}^{t}\left(\int_{0}^{z} m(\sigma) d \sigma\right) e^{-i z \gamma \lambda} b(i \delta \lambda) e^{i z \delta \lambda}\left(\int_{0}^{z} \tilde{m}(\sigma) d \sigma\right) d z+\tilde{R}_{t} \\
= & -\int_{0}^{t}\left(\int_{0}^{z} m(\sigma) d \sigma\right)(i \delta \lambda) e^{-i z \gamma \lambda} b e^{i z \delta \lambda}\left(\int_{0}^{z} \tilde{m}(\sigma) d \sigma\right) d z \\
& -\int_{0}^{t}\left(\int_{0}^{z} m(\sigma) d \sigma\right) e^{-i z \gamma \lambda}[b,(i \delta \lambda)] e^{i z \delta \lambda}\left(\int_{0}^{z} \tilde{m}(\sigma) d \sigma\right) d z+\tilde{R}_{t} .
\end{aligned}
$$


Again, we notice that the central term in the right hand-side of this expression is in $\mathscr{C}^{0}\left(\mathbb{R} ; \mathcal{L}\left(H^{s}(\Omega)\right)\right)$, since $[b,(i \delta \lambda)] \in \Psi_{\mathrm{phg}}^{0}(\Omega)$. As a consequence, we can write, for some $\tilde{\tilde{R}}_{t} \in \mathscr{C}^{0}\left(\mathbb{R} ; \mathcal{L}\left(H^{s}(\Omega)\right)\right)$,

$$
\begin{aligned}
-i \gamma \lambda A(t) & =-\int_{0}^{t}\left(\int_{0}^{z} m(\sigma) d \sigma\right)(i \delta \lambda) e^{-i z \gamma \lambda} b e^{i z \delta \lambda}\left(\int_{0}^{z} \tilde{m}(\sigma) d \sigma\right) d z+\tilde{\tilde{R}}_{t} \\
& =-i \delta \lambda A(t)-\int_{0}^{t}\left[\left(\int_{0}^{z} m(\sigma) d \sigma\right),(i \delta \lambda)\right] e^{-i z \gamma \lambda} b e^{i z \delta \lambda}\left(\int_{0}^{z} \tilde{m}(\sigma) d \sigma\right) d z+\tilde{\tilde{R}}_{t} .
\end{aligned}
$$

This gives

$$
i(\delta-\gamma) \lambda A(t)=-\int_{0}^{t}\left[\left(\int_{0}^{z} m(\sigma) d \sigma\right),(i \delta \lambda)\right] e^{-i z \gamma \lambda} b e^{i z \delta \lambda}\left(\int_{0}^{z} \tilde{m}(\sigma) d \sigma\right) d z+\tilde{\tilde{R}}_{t},
$$

and in particular $(\delta-\gamma) \lambda A(t) \in \mathscr{C}^{0}\left(\mathbb{R} ; \mathcal{L}\left(H^{s}(\Omega)\right)\right)$. This concludes the proof of Lemma A.2.

Proof of Lemma A.3. The definition of $\mathscr{F}$ directly gives $\mathscr{F} \in \mathscr{C}^{0}\left(0, T ; H^{s}(\Omega)\right.$ ) (with the associated estimate). We hence only have to check that $\lambda \mathscr{F} \in \mathscr{C}^{0}\left(0, T ; H^{s}(\Omega)\right.$ ). For this, we compute

$$
\begin{aligned}
-i \gamma \lambda \mathscr{F}(t) & =\int_{0}^{t}(-i \gamma \lambda) e^{-i z \gamma \lambda} b e^{i z \delta \lambda}\left(\int_{0}^{z} F(\sigma) d \sigma\right) d z \\
& =\left[e^{-i z \gamma \lambda} b e^{i z \delta \lambda}\left(\int_{0}^{z} F(\sigma) d \sigma\right)\right]_{0}^{t}-\int_{0}^{t} e^{-i z \gamma \lambda} b \partial_{z}\left[e^{i z \delta \lambda}\left(\int_{0}^{z} F(\sigma) d \sigma\right)\right] d z
\end{aligned}
$$

after an integration by parts. Furthermore

$$
\begin{aligned}
-\int_{0}^{t} e^{-i z \gamma \lambda} b \partial_{z}\left[e^{i z \delta \lambda}\left(\int_{0}^{z} F(\sigma) d \sigma\right)\right] d z= & -i \delta \int_{0}^{t} e^{-i z \gamma \lambda} b \lambda e^{i z \delta \lambda}\left(\int_{0}^{z} F(\sigma) d \sigma\right) d z \\
& -\int_{0}^{t} e^{-i z \gamma \lambda} b e^{i z \delta \lambda} F(z) d z \\
= & -i \delta \lambda \mathscr{F}(t)-i \delta \int_{0}^{t} e^{-i z \gamma \lambda}[b, \lambda] e^{i z \delta \lambda}\left(\int_{0}^{z} F(\sigma) d \sigma\right) d z \\
& -\int_{0}^{t} e^{-i z \gamma \lambda} b e^{i z \delta \lambda} F(z) d z
\end{aligned}
$$

Putting (A.2) in (A.3), we obtain

$$
\begin{aligned}
-i(\gamma-\delta) \lambda \mathscr{F}(t)= & e^{-i t \gamma \lambda} b e^{i t \delta \lambda}\left(\int_{0}^{t} F(\sigma) d \sigma\right)-i \delta \int_{0}^{t} e^{-i z \gamma \lambda}[b, \lambda] e^{i z \delta \lambda}\left(\int_{0}^{z} F(\sigma) d \sigma\right) d z \\
& -\int_{0}^{t} e^{-i z \gamma \lambda} b e^{i z \delta \lambda} F(z) d z
\end{aligned}
$$

The first and the last terms in (A.4) are in $\in \mathscr{C}^{0}\left(0, T ; H^{s}(\Omega)\right)$ as $z \rightarrow e^{i z \delta \lambda} \in \mathscr{C}^{0}\left(\mathbb{R} ; \mathcal{L}\left(H^{s}(\Omega)\right)\right)$. Finally the second term in (A.4) is also in $\mathscr{C}^{0}\left(0, T ; H^{s}(\Omega)\right)$ since $[b, \lambda] \in \Psi_{\mathrm{phg}}^{0}(\Omega)$. Estimate (A.1) comes from the $L^{\infty}\left(0, T ; H^{s}(\Omega)\right)$ estimate of (A.4).

\section{B Proofs of some technical results}

\section{B.1 Proof of Lemma 3.3}

The fact that the measures $\mu_{1}$ and $\mu_{2}$ are real and non-negative is a direct consequence of the first two equations of (3.7), tested on selfadjoint non-negative operators. Note also that the measures $\mu_{1}$ and $\mu_{2}$ are microlocal defect measures associated with the scalar sequences $\left(w_{1}^{k}\right)_{k \in \mathbb{N}}$ and $\left(w_{2}^{k}\right)_{k \in \mathbb{N}}$ respectively. 
Let us prove that $\operatorname{supp}\left(\mu_{2}\right) \subset \operatorname{Char}(P)$. Proving that $\operatorname{supp}\left(\mu_{1}\right) \subset \operatorname{Char}(P)$ follows the same steps. Recalling that the symbol $\tilde{\lambda} \in S_{\text {phg }}^{1}\left(T^{*} M ; \mathbb{C}\right)$ is defined in $(2.2)$, there exists $\tilde{\Lambda}_{-2} \in \Psi_{\text {phg }}^{-2}(M ; \mathbb{C})$ that satisfies $\sigma_{-2}\left(\tilde{\Lambda}_{-2}\right)=\tilde{\lambda}^{-2}$. Take $a \in S_{\mathrm{phg}}^{0}\left(T^{*} M_{T} ; \mathbb{C}\right)$ and $A \in \Psi_{\mathrm{phg}}^{0}\left(M_{T} ; \mathbb{C}\right)$ an associated operator. The second equation of (3.2) gives

$$
\left(A \tilde{\Lambda}_{-2} P w_{2}^{k}, w_{2}^{k}\right)_{L^{2}\left(M_{T} ; \mathbb{C}\right)}=-\left(A \tilde{\Lambda}_{-2} B w_{1}^{k}, w_{2}^{k}\right)_{L^{2}\left(M_{T} ; \mathbb{C}\right)} .
$$

Since $A \tilde{\Lambda}_{-2} B \in \Psi_{\mathrm{phg}}^{-1}\left(M_{T} ; \mathbb{C}\right)$, we have $A \tilde{\Lambda}_{-2} B w_{1}^{k} \rightarrow 0$ in $L^{2}\left(M_{T} ; \mathbb{C}\right)$ so that we obtain

$$
\left(A \tilde{\Lambda}_{-2} P w_{2}^{k}, w_{2}^{k}\right)_{L^{2}\left(M_{T} ; \mathbb{C}\right)} \rightarrow 0 .
$$

The second equation of (3.7) then gives

$$
0=\left\langle\mu_{2}, a \tilde{\lambda}^{-2} p\right\rangle_{S^{*} M_{T}}=\left\langle\mu_{2}, a p\right\rangle_{S^{*} M_{T}},
$$

which is satisfied for all $a \in S_{\text {phg }}^{0}\left(T^{*} M_{T} ; \mathbb{C}\right)$ if and only if $\operatorname{supp}\left(\mu_{2}\right) \subset \operatorname{Char}(P)$.

Now, let $a \in S_{\mathrm{phg}}^{0}\left(T^{*} M_{T} ; \mathbb{C}\right)$ and take $A \in \Psi_{\mathrm{phg}}^{0}\left(M_{T} ; \mathbb{C}\right)$ such that $\sigma_{0}(A)=a$. Then, we have

$$
\left(A w_{1}^{k}, A w_{2}^{k}\right)_{L^{2}\left(M_{T} ; \mathbb{C}\right)}=\left(A^{*} A w_{1}^{k}, w_{2}^{k}\right)_{L^{2}\left(M_{T} ; \mathbb{C}\right)} \rightarrow\left\langle\mu_{12},|a|^{2}\right\rangle_{S^{*} M_{T}} .
$$

Similarly, the Cauchy-Schwarz inequality gives

$$
\left|\left(A w_{1}^{k}, A w_{2}^{k}\right)_{L^{2}\left(M_{T} ; \mathbb{C}\right)}\right| \leq\left\|A w_{1}^{k}\right\|_{L^{2}\left(M_{T} ; \mathbb{C}\right)}\left\|A w_{2}^{k}\right\|_{L^{2}\left(M_{T} ; \mathbb{C}\right)} \rightarrow\left(\left\langle\mu_{1},|a|^{2}\right\rangle_{S^{*} M_{T}}\right)^{\frac{1}{2}}\left(\left\langle\mu_{2},|a|^{2}\right\rangle_{S^{*} M_{T}}\right)^{\frac{1}{2}} .
$$

This finally yields for all $a \in S_{\mathrm{phg}}^{0}\left(T^{*} M_{T} ; \mathbb{C}\right)$,

$$
\left|\left\langle\mu_{12},|a|^{2}\right\rangle_{S^{*} M_{T}}\right|^{2} \leq\left\langle\mu_{1},|a|^{2}\right\rangle_{S^{*} M_{T}}\left\langle\mu_{1},|a|^{2}\right\rangle_{S^{*} M_{T}}
$$

and hence $\operatorname{supp}\left(\mu_{12}\right) \subset \operatorname{supp}\left(\mu_{1}\right) \cap \operatorname{supp}\left(\mu_{2}\right)$.

Next, we define the operator

$$
\mathcal{Q}=\left(\begin{array}{ll}
P & B^{*} \\
0 & P
\end{array}\right) \in \Psi_{\mathrm{phg}}^{2}\left(M, \mathbb{C}^{2 \times 2}\right),
$$

where, $B^{*}$ denotes the adjoint of the operator $B$. Remark that, for all

$$
\mathcal{A}=\left(\begin{array}{ll}
A_{11} & A_{12} \\
A_{21} & A_{22}
\end{array}\right) \in \Psi_{\text {phg }}^{-1}\left(M_{T}, \mathbb{C}^{2 \times 2}\right),
$$

we have

$$
(\mathcal{A P}-\mathcal{Q A})=\left(\begin{array}{ll}
{\left[A_{11}, P\right]+A_{12} B-B^{*} A_{21}} & {\left[A_{12}, P\right]-B^{*} A_{22}} \\
{\left[A_{21}, P\right]+A_{22} B} & {\left[A_{22}, P\right]}
\end{array}\right) \in \Psi_{\mathrm{phg}}^{0}\left(M_{T}, \mathbb{C}^{2 \times 2}\right) .
$$

Hence, we have

$$
(\mathcal{A P}-\mathcal{Q A}) W^{k} \in L^{2}\left(M_{T} ; \mathbb{C}^{2}\right)
$$

together with

$$
\mathcal{A P} W^{k}=0 .
$$

In particular, these last two identities yield

$$
\mathcal{Q} \mathcal{A} W^{k} \in L^{2}\left(M_{T} ; \mathbb{C}^{2}\right) .
$$

For smooth data $\left.W^{k}\right|_{t=0}$, we can integrate by parts and have

$$
\left(\mathcal{Q} \mathcal{A} W^{k}, W^{k}\right)_{L^{2}\left(M_{T} ; \mathbb{C}^{2}\right)}=\left(\mathcal{A} W^{k}, \mathcal{P} W^{k}\right)_{L^{2}\left(M_{T} ; \mathbb{C}^{2}\right)}=0
$$


since the operator $\mathcal{A}$ has a kernel with compact support (and hence the boundary terms at times 0 and $T$ vanish). A density argument together with (B.2) then gives $\left(\mathcal{Q A} W^{k}, W^{k}\right)_{L^{2}\left(M_{T} ; \mathbb{C}^{2}\right)}=0$ for all $W^{k} \in L^{2}\left(M_{T} ; \mathbb{C}^{2}\right)$. Hence, we have

$$
0=\left((\mathcal{A P}-\mathcal{Q A}) W^{k}, W^{k}\right)_{L^{2}\left(M_{T} ; \mathbb{C}^{2}\right)} \cdot
$$

As a consequence of (3.6), (B.3) and (B.1), we obtain

$$
0=\int_{S^{*} M_{T}} \operatorname{tr}\left\{\left(\begin{array}{ll}
\frac{1}{i}\left\{a_{11}, p\right\}+b|\eta|_{x}\left(a_{12}-a_{21}\right) & \frac{1}{i}\left\{a_{12}, p\right\}-b|\eta|_{x} a_{22} \\
\frac{1}{i}\left\{a_{21}, p\right\}+b|\eta|_{x} a_{22} & \frac{1}{i}\left\{a_{22}, p\right\}
\end{array}\right)\left(\begin{array}{ll}
\mu_{1} & \mu_{12} \\
\bar{\mu}_{12} & \mu_{2}
\end{array}\right)(d \rho)\right\},
$$

with $a_{j l}=\sigma_{-1}\left(A_{j l}\right), j, l=1,2$. Since the application $A \rightarrow \sigma_{-1}(A)$ is from $\Psi_{\mathrm{phg}}^{-1}\left(M_{T}\right)$ onto $S_{\mathrm{phg}}^{-1}\left(T^{*} M_{T} ; \mathbb{C}\right)$, this is equivalent to

$$
\left\{\begin{array}{l}
\left\langle\mu_{1}, \frac{1}{i}\left\{a_{11}, p\right\}\right\rangle_{S^{*} M_{T}}=0 \\
\left\langle\mu_{2}, \frac{1}{i}\left\{a_{22}, p\right\}\right\rangle_{S^{*} M_{T}}+\left\langle 2 i \operatorname{Im}\left(\mu_{12}\right), b|\eta|_{x} a_{22}\right\rangle_{S^{*} M_{T}}=0 \\
\left\langle\mu_{1}, b|\eta|_{x}\left(a_{12}-a_{21}\right)\right\rangle_{S^{*} M_{T}}+\left\langle\mu_{12}, \frac{1}{i}\left\{a_{21}, p\right\}\right\rangle_{S^{*} M_{T}}+\left\langle\bar{\mu}_{12}, \frac{1}{i}\left\{a_{12}, p\right\}\right\rangle_{S^{*} M_{T}}=0
\end{array}\right.
$$

for any $a_{11}, a_{22}, a_{12}, a_{21} \in S_{\mathrm{phg}}^{-1}\left(T^{*} M_{T} ; \mathbb{C}\right)$. Taking successively $a_{21}=a_{12}$ and $a_{21}=-a_{12}$ in the last identity of (B.4) yields

$$
\left\langle\operatorname{Re}\left(\mu_{12}\right), \frac{1}{i}\left\{a_{12}, p\right\}\right\rangle_{S^{*} M_{T}}=0 \quad \text { and } \quad\left\langle\mu_{1}, b|\eta|_{x} a_{12}\right\rangle_{S^{*} M_{T}}-\left\langle\operatorname{Im}\left(\mu_{12}\right),\left\{a_{12}, p\right\}\right\rangle_{S^{*} M_{T}}=0 .
$$

These equations together with the first two identities of (B.4) give (3.8), which concludes the proof of the first part of the lemma.

Moreover, (3.4) yields

$$
0=\lim _{k \rightarrow \infty}\left(\left|b_{\omega}\right|^{2} w_{2}^{k}, w_{2}^{k}\right)_{L^{2}\left(M_{T} ; \mathbb{C}\right)}=\left\langle\mu_{2},\left|b_{\omega}\right|^{2}\right\rangle_{S^{*} M_{T}},
$$

which directly gives $\pi\left(\operatorname{supp}\left(\mu_{2}\right)\right) \cap((0, T) \times \omega)=\emptyset$ as $\mu_{2} \geq 0$ and $\left\{b_{\omega}>0\right\}=\omega$. This concludes the proof of the second part of the lemma as $\operatorname{supp}\left(\mu_{12}\right) \subset \operatorname{supp}\left(\mu_{2}\right)$.

\section{B.2 Proof of Lemma 4.3}

A proof of this lemma can be found in the semiclassical setting in [Bur97a, Section 4.2] (see also [BG97]). We can parametrize the bicharacteristic $\Gamma$ as

$$
\Gamma \cap \pi^{-1}\left(M_{T}\right)=\left\{\phi_{s}\left(\rho_{0}\right), s \in(0, T)\right\},
$$

with

$$
\rho_{0}=\left(0, x_{0}, \tau_{0}, \eta_{0}\right) \in \Gamma \cap\{t=0\} .
$$

We have $\tau_{0}^{2}=\left|\eta_{0}\right|_{x}^{2}$. Let us assume that $\tau_{0}>0$. The case $\tau_{0}<0$ can be treated similarly. We set

$$
\nu_{0}=\left(x_{0}, \frac{\eta_{0}}{\left|\eta_{0}\right|_{x}}\right) \in S^{*} \Omega
$$

There exists a local chart $\left(U_{\kappa}, \kappa\right)$ of $\Omega$ such that $x_{0} \in U_{\kappa}$. We denote by $\left(y_{0}, \xi_{0}\right)$ the coordinates of $\nu_{0}$ in this chart.

We choose $\psi \in \mathscr{C}_{c}^{\infty}\left(\mathbb{R}^{n}\right)$ such that $\operatorname{supp}(\psi) \subset \kappa\left(U_{\kappa}\right)$, and $\psi=1$ in a neighborhood of $y_{0}$. Next we define

$$
v^{k}(y)=C_{0} k^{\frac{n}{4}} e^{i k \varphi(y)} \psi(y), \quad \text { with } \varphi(y)=y \cdot \xi_{0}+i\left(y-y_{0}\right)^{2} \text { and } C_{0}>0 .
$$


Now, we set

$$
w_{0}^{k}=\kappa^{*} v^{k} \in \mathscr{C}_{c}^{\infty}(\Omega) .
$$

We have $w_{0}^{k} \rightarrow 0$ in $L^{2}(\Omega), \lim _{k \rightarrow \infty}\left\|w_{0}^{k}\right\|_{L^{2}(\Omega)}=1$ for an appropriate choice of $C_{0}$. Moreover, a classical computation on $\left(v^{k}\right)_{k \in \mathbb{N}}$ shows that $\left(w_{0}^{k}\right)_{k \in \mathbb{N}}$ is pure and admits the microlocal defect measure $m=\delta_{(x, \eta)=\nu_{0}}$.

We set $w_{1}^{k}=i \lambda w_{0}^{k} \in$ and let $w^{k}$ be the solution of

$$
\begin{cases}P w^{k}=0 & \text { in }(0, T) \times \Omega \\ \left.\left(w^{k}, \partial_{t} w^{k}\right)\right|_{t=0}=\left(w_{0}^{k}, w_{1}^{k}\right) & \text { on } \Omega\end{cases}
$$

For the sequence $\left(w^{k}\right)_{k \in \mathbb{N}},(4.4)$ is satisfied. We recall that $L_{+}, L_{-}, \lambda \ldots$ are defined in Section 2.1. Setting $z^{k}=L_{+} w^{k}$, we have $L_{-} z^{k}=0$ and

$$
\left.z^{k}\right|_{t=0}=\left.\left(\frac{1}{i} \partial_{t} w^{k}-\lambda w^{k}\right)\right|_{t=0}=\frac{1}{i} w_{1}^{k}-\lambda w_{0}^{k}=0 .
$$

Therefore, $z^{k}=0$ in $\mathbb{R}^{+} \times \Omega$, i.e. $w^{k}$ solves the first-order equation

$$
\begin{cases}L_{+} w^{k}=0 & \text { in }(0, T) \times \Omega \\ \left.w^{k}\right|_{t=0}=w_{0}^{k} & \text { on } \Omega\end{cases}
$$

System (B.7) is well-posed, and the sequence $\left(w^{k}\right)_{k \in \mathbb{N}}$ is bounded in $L^{2}\left(M_{T}\right)$ (see [Hör85, Section 23.1]), weakly converging to zero and hence admits a microlocal defect measure $\mu$ (up to a subsequence).

Now, we prove that $\operatorname{supp}(\mu) \subset \Gamma$. Since $w^{k}$ solves (B.6), the measure $\mu$ is preserved along $\Gamma$ which is also a bicharacteristic curve of $\ell_{+}=\tau-|\eta|_{x}$. Hence, it suffices to prove that any point $\left(0, x_{1}, \tau_{1}, \eta_{1}\right) \in \operatorname{Char}(P) \cap S^{*} M$ different from $\left(0, x_{0}, \tau_{0}, \eta_{0}\right)$ is not in $\operatorname{supp}(\mu)$.

Let $a \in S_{\text {phg }}^{0}\left(T^{*} \Omega ; \mathbb{C}\right)$ be a zero-order homogeneous symbol, with compact support near $x_{1}$, such that $a=1$ in a conic neighborhood of $\left(x_{1}, \eta_{1}\right)$ and $a=0$ in a conic neighborhood of $\left(x_{0}, \eta_{0}\right)$. Taking $A \in \Psi_{\mathrm{phg}}^{0}(\Omega ; \mathbb{C})$ such that $\sigma_{0}(A)=a$, we have

$$
A w_{0}^{k} \rightarrow 0 \quad \text { in } L^{2}(\Omega),
$$

since $m=\delta_{(x, \eta)=\nu_{0}}$. We shall now use the flows $\phi_{s}^{+}$and $\varphi_{s}^{+}$, defined in Section 2.2.

We define the tangential polyhomogeneous symbol ${ }^{5}$

$$
q(t, x, \eta)=a\left(\varphi_{-t}^{+}(x, \eta)\right) \in S_{\mathcal{T}}^{0}\left((-T, T) \times T^{*} \Omega ; \mathbb{C}\right),
$$

which satisfies

$$
\left\{\begin{array}{l}
H_{\ell_{+}} q=0 \\
q(0, x, \eta)=a(x, \eta)
\end{array}\right.
$$

We denote by $\Psi_{\mathcal{T}}^{m}((-T, T) \times \Omega ; \mathbb{C})$ the set of tangential operators of order $m$. We take $Q \in$ $\Psi_{\mathcal{T}}^{0}((-T, T) \times \Omega ; \mathbb{C})$ such a tangential operator satisfying $\sigma_{0}(Q)=q$. The commutator $\left[L_{+}, Q\right]$ satisfies $\left[L_{+}, Q\right] \in \Psi_{\mathcal{T}}^{0}((-T, T) \times \Omega ; \mathbb{C})$ with principal symbol $\frac{1}{i}\left\{\ell_{+}, q\right\}=\frac{1}{i} H_{\ell_{+}} q=0$. Hence, we have $\left[L_{+}, Q\right] \in \Psi_{\mathcal{T}}^{-1}((-T, T) \times \Omega ; \mathbb{C})$. Now, we compute

$$
\left\{\begin{array}{l}
L_{+} Q w^{k}=\left[L_{+}, Q\right] w^{k} \rightarrow 0 \text { in } L^{2}\left(M_{T}\right) \\
\left.Q w^{k}\right|_{t=0}=A w_{0}^{k} \rightarrow 0 \text { in } L^{2}(\Omega)
\end{array}\right.
$$

Applying then the hyperbolic energy inequality, to this first-order system, we obtain

$$
\left\|Q w^{k}\right\|_{L^{\infty}\left(0, T ; L^{2}(\Omega)\right)} \leq C\left(\left\|A w_{0}^{k}\right\|_{L^{2}(\Omega)}+\left\|\left[L_{+}, Q\right] w^{k}\right\|_{L^{1}\left(0, T ; L^{2}\left(M_{T}\right)\right)}\right) \rightarrow 0 .
$$

\footnotetext{
${ }^{5}$ For tangential symbols $S_{\mathcal{T}}^{m}\left((-T, T) \times T^{*} \Omega ; \mathbb{C}\right)$ and associated operators $\Psi_{\mathcal{T}}^{m}((-T, T) \times \Omega ; \mathbb{C})$, we refer to $[$ Hör85, page 94 (bottom)]. Here, $t$ stands for the parameter upon which the symbols depend.
} 
Finally, let $r \in S_{\mathrm{phg}}^{0}\left(T^{*} M ; \mathbb{C}\right)$ be such that $r=1$ in a neighborhood of $\left(0, x_{1}, \tau_{1}, \eta_{1}\right), r=0$ for $|\eta|_{x}<\frac{|\tau|}{2}$, and $r$ has a compact support in the time variable $t$, included in $\left(-\frac{T}{2}, \frac{T}{2}\right)$. Taking $R \in$ $\Psi_{\mathrm{phg}}^{0}(M ; \mathbb{C})$ such that $\sigma_{0}(R)=r$ the operator $R Q$ is in the class $\Psi_{\mathrm{phg}}^{0}(M ; \mathbb{C})$, according to [Hör85, Theorem 18.1.35]. Moreover, $R Q$ is elliptic at $\left(0, x_{1}, \tau_{1}, \eta_{1}\right)$ since $r\left(0, x_{1}, \tau_{1}, \eta_{1}\right) q\left(0, x_{1}, \eta_{1}\right)=1$ and we have

$$
\left\|R Q w^{k}\right\|_{L^{2}\left(M_{T}\right)} \leq C\left\|Q w^{k}\right\|_{L^{2}\left(M_{T}\right)} \rightarrow 0 .
$$

As a consequence, $\left(0, x_{1}, \tau_{1}, \eta_{1}\right) \notin \operatorname{supp}(\mu)$. The invariance of $\mu$ along the bicharacteristic flow finally gives $\operatorname{supp}(\mu) \subset \Gamma$, which concludes the proof of Lemma 4.3.

Acknowledgment. Part of this research was carried out at Institut Henri Poincaré (Paris, France) during the "Control of Partial and Differential Equations and Applications" program in the Fall 2010. The authors wish to thank Institut Henri Poincaré for providing a very stimulating environment. The first author was supported by the Tunisian Ministery for Scientific Research and Technology within the LAB-STI 02 program. The authors wish to thank Camille Laurent for very interesting discussions on the subject of this article.

\section{References}

[AB03] F. Alabau-Boussouira. A two-level energy method for indirect boundary observability and controllability of weakly coupled hyperbolic systems. SIAM J. Control Optim., 42:871-906, 2003.

[AB12] F. Alabau-Boussouira. Controllability of cascade coupled systems of multi-dimensional evolution pde's by a reduced number of controls. preprint, 2012.

[ABL11] F. Alabau-Boussouira and M. Léautaud. Indirect controllability of locally coupled systems under geometric conditions. C. R. Math. Acad. Sci. Paris, 349(7-8):395-400, 2011.

[ABL12] F. Alabau-Boussouira and M. Léautaud. Indirect controllability of locally coupled wave-type systems and applications. to appear in J. Math. Pures Appl., 2012.

[AKBGBdT11] F. Ammar-Khodja, A. Benabdallah, M. González-Burgos, and L. de Teresa. Recent results on the controllability of linear coupled parabolic problems: a survey. Math. Control Relat. Fields, 1(3):267-306, 2011.

[AKS62] N. Aronszajn, A. Krzywicki, and J. Szarski. A unique continuation theorem for exterior differential forms on Riemannian manifolds. Ark. Mat., 4:417-453, 1962.

[Aro57] N. Aronszajn. A unique continuation theorem for solutions of elliptic partial differential equations or inequalities of second order. J. Math. Pures Appl. (9), 36:235-249, 1957.

[BG97] N. Burq and P. Gérard. Condition nécessaire et suffisante pour la contrôlabilité exacte des ondes. C. R. Acad. Sci. Paris Sér. I Math., 325(7):749-752, 1997.

[BG02] N. Burq and P. Gérard. Contrôle optimal des équations aux dérivées partielles. Cours de l'Ecole Polytechnique, 2002.

[BL01] N. Burq and G. Lebeau. Mesures de défaut de compacité, application au système de Lamé. Ann. Sci. École Norm. Sup. (4), 34(6):817-870, 2001.

[BLR92] C. Bardos, G. Lebeau, and J. Rauch. Sharp sufficient conditions for the observation, control, and stabilization of waves from the boundary. SIAM J. Control Optim., 30:1024-1065, 1992.

[Bur97a] N. Burq. Contrôlabilité exacte des ondes dans des ouverts peu réguliers. Asymptot. Anal., 14(2):157-191, 1997.

[Bur97b] N. Burq. Mesures semi-classiques et mesures de défaut. Astérisque, (245):Exp. No. 826, 4, 167-195, 1997. Séminaire Bourbaki, Vol. 1996/97.

[CP82] J. Chazarain and A. Piriou. Introduction to the Theory of Linear Partial Differential Equations. North-Holland, Amsterdam, 1982.

[Dág06] R. Dáger. Insensitizing controls for the 1-D wave equation. SIAM J. Control Optim., 45(5):1758-1768, 2006.

[Den82] N. Dencker. On the propagation of polarization sets for systems of real principal type. $J$. Funct. Anal., 46(3):351-372, 1982. 
[DL09] B. Dehman and G. Lebeau. Analysis of the HUM control operator and exact controllability for semilinear waves in uniform time. SIAM J. Control Optim., 48(2):521-550, 2009.

[DR77] S. Dolecki and D.L. Russell. A general theory of observation and control. SIAM J. Control Optim., 15(2):185-220, 1977.

[ET74] I. Ekeland and R. Temam. Analyse convexe et problèmes variationnels. Dunod-GauthierVillars, Paris, 1974.

[Gér91] P. Gérard. Microlocal defect measures. Comm. Partial Differential Equations, 16(11):17611794, 1991.

[Hör85] L. Hörmander. The Analysis of Linear Partial Differential Operators, volume III. SpringerVerlag, 1985. Second printing 1994.

[Hör90] L. Hörmander. The Analysis of Linear Partial Differential Operators, volume I. SpringerVerlag, Berlin, second edition, 1990.

[Hör94] L. Hörmander. The analysis of linear partial differential operators. IV, volume 275 of Grundlehren der Mathematischen Wissenschaften. Springer-Verlag, Berlin, 1994. Fourier integral operators, Corrected reprint of the 1985 original.

[Léa10] M. Léautaud. Spectral inequalities for non-selfadjoint elliptic operators and application to the null-controllability of parabolic systems. J. Funct. Anal., 258:2739-2778, 2010.

[Leb96] G. Lebeau. Équation des ondes amorties. In Algebraic and geometric methods in mathematical physics (Kaciveli, 1993), volume 19 of Math. Phys. Stud., pages 73-109. Kluwer Acad. Publ., Dordrecht, 1996.

[Ler10] N. Lerner. Metrics on the phase space and non-selfadjoint pseudo-differential operators. Birkhäuser Verlag, Basel, 2010.

[Lio88] J.-L. Lions. Contrôlabilité exacte, perturbations et stabilisation de systèmes distribués. Tome 1, volume 8 of Recherches en Mathématiques Appliquées. Masson, Paris, 1988.

[Lio90] J.-L. Lions. Quelques notions dans l'analyse et le contrôle de systèmes à données incomplètes. In Proceedings of the XIth Congress on Differential Equations and Applications/First Congress on Applied Mathematics (Spanish) (Málaga, 1989), pages 43-54, Málaga, 1990. Univ. Málaga.

[LL11] J. Le Rousseau and G. Lebeau. On Carleman estimates for elliptic and parabolic operators. applications to unique continuation and control of parabolic equations. ESAIM Control Optim. Calc. Var., DOI:10.1051/cocv/2011168, 2011.

[LN10] G. Lebeau and M. Nodet. Experimental study of the HUM control operator for linear waves. Experiment. Math., 19(1):93-120, 2010.

[LZ99] G. Lebeau and E. Zuazua. Decay rates for the three-dimensional linear system of thermoelasticity. Arch. Rational Mech. Anal., 148:179-231, 1999.

[Paz83] A. Pazy. Semigroups of Linear Operators and Applications to Partial Differential Equations. Springer-Verlag, New York, 1983.

[RdT11] L. Rosier and L. de Teresa. Exact controllability of a cascade system of conservative equations. C. R. Math. Acad. Sci. Paris, 349(5-6):291-296, 2011.

[RT74] J. Rauch and M. Taylor. Exponential decay of solutions to hyperbolic equations in bounded domains. Indiana Univ. Math. J., 24:79-86, 1974.

[See67] R. T. Seeley. Complex powers of an elliptic operator. In Singular Integrals (Proc. Sympos. Pure Math., Chicago, Ill., 1966), pages 288-307. Amer. Math. Soc., Providence, R.I., 1967.

[Shu01] M. A. Shubin. Pseudodifferential Operators and Spectral Theory. Springer-Verlag, Berlin Heidelberg, second edition, 2001.

[Tar90] L. Tartar. $H$-measures, a new approach for studying homogenisation, oscillations and concentration effects in partial differential equations. Proc. Roy. Soc. Edinburgh Sect. A, 115(34):193-230, 1990.

[Tay81] M. E. Taylor. Pseudodifferential Operators. Princeton University Press, Princeton, New Jersey, 1981.

[Tay91] M. E. Taylor. Pseudodifferential Operators and Nonlinear PDE. Birkhäuser, Boston, 1991. 
[Teb08] L. Tebou. Locally distributed desensitizing controls for the wave equation. C. R. Math. Acad. Sci. Paris, 346(7-8):407-412, 2008.

[Zui83] C. Zuily. Uniqueness and nonuniqueness in the Cauchy problem, volume 33 of Progress in Mathematics. Birkhäuser Boston Inc., Boston, MA, 1983. 